\title{
New insights in the development of pressure ulcers
}

\author{
Citation for published version (APA):
}

de Wert, L. (2019). New insights in the development of pressure ulcers. [Doctoral Thesis, Maastricht University]. Maastricht University. https://doi.org/10.26481/dis.20190222lw

Document status and date:

Published: 01/01/2019

DOI:

10.26481/dis.20190222Iw

Document Version:

Publisher's PDF, also known as Version of record

\section{Please check the document version of this publication:}

- A submitted manuscript is the version of the article upon submission and before peer-review. There can be important differences between the submitted version and the official published version of record.

People interested in the research are advised to contact the author for the final version of the publication, or visit the DOI to the publisher's website.

- The final author version and the galley proof are versions of the publication after peer review.

- The final published version features the final layout of the paper including the volume, issue and page numbers.

Link to publication

\footnotetext{
General rights rights.

- You may freely distribute the URL identifying the publication in the public portal. please follow below link for the End User Agreement:

www.umlib.nl/taverne-license

Take down policy

If you believe that this document breaches copyright please contact us at:

repository@maastrichtuniversity.nl

providing details and we will investigate your claim.
}

Copyright and moral rights for the publications made accessible in the public portal are retained by the authors and/or other copyright owners and it is a condition of accessing publications that users recognise and abide by the legal requirements associated with these

- Users may download and print one copy of any publication from the public portal for the purpose of private study or research.

- You may not further distribute the material or use it for any profit-making activity or commercial gain

If the publication is distributed under the terms of Article $25 \mathrm{fa}$ of the Dutch Copyright Act, indicated by the "Taverne" license above, 
New insights in the development of

$$
\text { pressure ulcers }
$$


(C) LA de Wert, Maastricht 2019, All rights reserved

Cover: Art Collart

Layout: Tiny Wouters Lenssen

Production by: proefschriftmaken.nl

ISBN 978-94-6380-232-1

Publication of this thesis was financially supported by Maastricht University, Department of Surgery of the Maastricht University Medical Centre. Additional funding was obtained by the Kootstra Talent Fellowship, Maastricht University Medical Centre. 


\title{
New insights in the development of
}

\section{pressure ulcers}

\author{
PROEFSCHRIFT \\ ter verkrijging van de graad van doctor aan de Universiteit Maastricht, \\ op gezag van de Rector Magnificus, prof. dr. Rianne M. Letschert, \\ volgens het besluit van het College van Decanen, \\ in het openbaar te verdedigen \\ op vrijdag 22 februari 2019 om 10.00 uur
}

door

Luuk Albert de Wert 


\section{Promotoren}

Prof. dr. N.D. Bouvy

Prof. dr. M. Poeze

\section{Beoordelingscommissie}

Prof. dr. L.W. van Rhijn (voorzitter)

Dr. B. Havekes

Prof. dr. I.C. Heyligers

Dr. S.W. Nienhuijs (Catharina ziekenhuis, Eindhoven)

Prof. dr. M.R.H.M. van Sambeek (Technische Universiteit Eindhoven)

Prof. dr. P.M. Steijlen 


\section{Contents}

$\begin{array}{lll}\text { Chapter } 1 \quad \text { General Introduction } & 7\end{array}$

Chapter 2 The cutaneous microbiome in hospitalized patients with 21 pressure ulcers

Chapter 3 A new method to evaluate the effects of shear on the skin 39

Chapter 4 The effect of shear on skin viability in patients with 55 type 2 diabetes

Chapter 5 Cytokine IL-1 $\alpha$ and lactate as a markers for tissue damage in spine board immobilization: A prospective randomized open-label crossover trial

Chapter 6 Improving the effect of shear on skin viability with wound dressings

Chapter 7 The prevention of sacral pressure ulcers in high- risk patients with the use of a sacral wound dressing: a randomized clinical trial

Chapter 8 General discussion

Nederlandse samenvatting

Valorisation addendum

Dankwoord

Curriculum Vitae 



\section{Chapter 1}

General introduction 



\section{Introduction}

A pressure ulcer, also known as decubitus ulcers or bedsores, is localised injury to the skin and/ or underlying tissue usually over a bony prominence, as a result of loading on the skin. Pressure ulcers can develop anywhere on the body, but are most likely to appear on skin area's exposed to high mechanical loading, so the sacral area, heel, elbow, ischial and trochanteric areas are affected most. Pressure ulcers occur mostly in people who are unable to move and therefore bound to a bed or (wheel) chair. However, several other risk factors ${ }^{1-3}$ have been identified such as: high age; poor nutritional status; sensory impairment; diabetes mellitus; bowel incontinence; smoking, paraplegia; or medication affecting skin blood flow.

According to the NPUAP/ EPUAP, pressure ulcers can be classified in to different categories (Table 1.1) ${ }^{4}$.

Table 1.1 Classification of pressure ulcers

\begin{tabular}{ll}
\hline Category & Explanation \\
\hline II & Non-blanchable erythema; intact skin with non-blanchable \\
& erythema. \\
Partial thickness skin loss; loss of dermis. Open shallow ulcer & with a red pink wound bed. Shiny or dry ulcer. \\
III & Full thickness skin loss; fat may be visible. Bone, tendon, \\
& muscle are not exposed. May include undermining and \\
& tunnelling. \\
IV & Full thickness tissue loss; with exposed bone, tendon or \\
& muscle. Often undermining and tunnelling. Can extend to \\
& muscle/ supporting tissue (e.g. fascia, tendon, joint). \\
& Osteomyelitis is possible. \\
Depth unknown: base covered with slough (yellow, tan, gray, \\
green, brown) and/ or eschar (tan, brown, black). Category \\
cannot determined. \\
Depth unknown; purple or maroon or discoloured intact skin \\
or blood filled blister due to damage of underlying tissue. May \\
include a thin blister over a dark wound bed, May evolve and \\
become covered by eschar.
\end{tabular}

The first description of the age-old problem of pressure ulcers dates back to Hippocrates in $400 \mathrm{BC}^{5}$. However, the problem of pressure ulcers is possibly as old as mankind, as they have been documented in autopsy reports on 5000-year-old Egyptian mummies. It was not until the Renaissance that pressure ulcers were associated with mechanical loading and nutritional status. In a case report Ambroise Paré (1510-1590) ${ }^{6}$, an influential French surgeon, reported the successful treatment of a pressure ulcer with nutritional support and pressure relief. Some 300 years later, a pressure ulcer was referred as decubitus ominosus, by Jean Martin Charcot $(1825-1893)^{7}$, which was linked to the worse prognostic outcome when a pressure ulcer was present. 
Currently, prognostic outcomes have improved a lot, however, this major problem is still associated with a high mortality, morbidity, pain and a lower quality of life. In addition, the financial impact of the costs associated with the prevention and treatment of pressure ulcers is extremely high, making it the third most expensive disease after cancer and cardiovascular diseases ${ }^{8,9}$.

\section{Mechanism of the development of pressure ulcers by both pressure and shear}

Pressure ulcers occur as a result of prolonged mechanical loading on the skin by pressure, or pressure in combination with shear ${ }^{10,11}$. Pressure is the normal perpendicular force per area and compresses the skin and underlying tissue. Shear forces are forces that act parallel to the skin and normally occur between the skin and interface of a support surface, such that there is deformation of tissue in parallel planes.

Mechanical loading of the skin causes blood vessels to occlude which, in turn, leads to ischemia, a change in metabolism, a hypoxic state of tissue and the accumulation of toxic waste products and metabolites and eventually cell death ${ }^{12,13}$. Despite the fact that this process can be accelerated by an impaired lymphatic and interstitial flow: it still takes several hours to develop ${ }^{14,15}$.

Normally, there will be an increase in cutaneous blood flow when mechanical loading on the skin is relieved. This increase in post load cutaneous blood flow is called 'reactive hyperaemia' and is an reaction on tissue ischemia, the accumulation of metabolites, necrosis or cell death ${ }^{16}$. It is considered an important physiological response to loading on the skin to restore any nutritional or oxygen deficit. Fromy and colleagues ${ }^{17}$ demonstrated in a mice model that when a normal reactive hyperaemic response is present, the skin will be better protected against pressure injury. Other studies $^{18}$ showed that the ability to develop reactive hyperaemia has been negatively affected in patients with diabetes mellitus ${ }^{19,20}$ or the elderly.

Pressure ulcers can develop very rapidly, so it is unlikely that ischemia can be the only cause of pressure ulcers. Completely ischemic skin and muscle have been shown to survive for more than two hours outside a body after plastic surgical free flap transfer. Recently, the Technical University of Eindhoven revealed another pathway that can lead to the development of cellular damage after loading on the skin. They proved that high mechanical loading and tissue deformation could activate a much faster process, which is called direct deformation damage. In this case, the cellular membrane and cytoskeleton becomes disrupted and the first signs of cellular damage become already visible within ten minutes. The basic assumption that pressure ulcers appear as a result of prolonged ischemia of the skin and underlying tissue is therefore outdated, because pressure ulcers are most likely to develop by this direct deformation damage. The accumulation of metabolic waste products and tissue 
ischemia, on the other hand, act mostly as an amplifier on skin and underlying tissue damage $^{21}$.

When shear is added to pressure, it could lead to enhanced internal deformation and earlier occlusion of blood vessels ${ }^{22,23}$, but reliable measurement in this context remains a difficult challenge.

\section{Prevention of pressure ulcers}

Currently, pressure ulcer preventive strategies are based on mechanical load management in patients at risk for the development of pressure ulcers. Patients at risk for pressure ulcers should be repositioned every 2-4 hours to restore blood flow in compressed tissue to avoid ischemic damage ${ }^{24}$. To reduce the effect of direct deformation damage, many pressure reducing devices have been developed ${ }^{25,26}$. Specialized foam mattresses, air fluidized bed systems, alternating pressure mattresses, mattress overlays and even specialized sheep skin are just a few examples of devices that are used to prevent pressure ulcer development in patients. Although these preventive measures have proven to be effective, a critical economic analysis is always needed before preventive measures could be implemented in standard hospital care. For example, the daily costs associated with pressure ulcer prevention can be up to $€ 85,57$ per patient per day ${ }^{27}$, while turning a patient every 2-4 hours can be very labour intensive as well. Therefore, it is important focus on preventive measures for patients who are actually at risk for the development of pressure ulcers, otherwise it cannot be considered cost-effective.

Another important contributory factor in pressure ulceration is the presence of shear. Just recently, prophylactic wound dressings are applied to areas on skin exposed to pressure and shear in order to prevent the development of pressure ulcers. It is believed that wound dressings can influence the microclimate of the skin, and more importantly, absorb shear forces. Several ex vivo studies demonstrated promising results $^{28-30}$ and demonstrated that wound dressings can reduce internal shear in tissues. In addition, clinical studies ${ }^{31-34}$ have shown a decrease in pressure ulcer incidence when dressings were used as addition to current pressure ulcer preventing strategies. However, these studies are compromised by a high risk of bias ${ }^{35}$ or weak methodological design ${ }^{36}$.

\section{Risk assessment}

\section{Risk assessment scales}

To implement pressure ulcer preventive strategies, reliable risk assessment is needed to identify patients at risk for the development of pressure ulcers. Several risk 
assessment scales, which are normally some kind of risk questionnaires, have been developed and validated to accomplish this ${ }^{37}$.

Especially the Braden, Norton and Waterlow are commonly used scales ${ }^{38}$, however, several other scales have been developed to predict pressure ulcer risk in patients ${ }^{39}$. Although risk assessment scales are widely used in hospitals and nursing homes, the predictive values associated with these scales are low, so pressure ulcer risk is therefore frequently predicted wrong ${ }^{38}$. Indeed, many patients will receive preventive care who are actually not at risk for the development of pressure ulcers, while patients at high risk might not receive preventive measures at all. Hence, risk assessment scales are not sufficient to predict pressure ulcer risk, so a more reliable risk method should be developed to counter this problem.

\section{Biochemical markers}

Damage-associated molecular patterns (DAMPs) are molecules that can initiate the immune system are released in the extracellular space when cells are damages or injured to initiate an inflammatory response. Well known DAMPs are IL-1 $\alpha, \mathrm{IL}-33$, high-mobility group box 1 (HMGB1), ATP, DNA strains, uric acid ${ }^{40}$. DAMPs have no inflammatory activity inside the cell, but when they are released in the extracellular matrix, they can bind to receptors on expressed on the cell wand and activate signalling pathways in order to stimulate the production of pro-inflammatory cytokines such as: IL-6, IL-8, Fibroblast Growth factor (FGF), Epidermal Growth factor (EGF), and Tumour Necrosis Factor- $\alpha$ (TNF- $\alpha$ ). These cytokines are essential in the initiation of inflammation and wound healing ${ }^{41,42}$.

Although DAMPs and cytokines seems to be promising biomarkers to detect skin damage before it is even visible for the human eye, a few problems needs to be addressed before they could actually be used as objective biomarkers to predict pressure ulcer risk in patients. For example, it is hard to it is hard to optimize a specific cut-off score for individual cytokines, because of a high inter-individual variability. Ideally, a larger set of biomarkers consisting cytokines, metabolites and other parameters could be utilized to predict individual pressure ulcer risk.

Two promising studies focussed on the change in electrical properties of skin tissue to predict pressure ulcer risk. A study performed by Swisher and colleagues ${ }^{43}$ demonstrated a change in electrical impedance caused by a loss of cellular integrity in a rat model. Electrical impedance was measured non-invasively with a flexible electrode described as 'smart bandage'. In the future, this 'smart bandage' might be used as an tool for pressure ulcers risk assessment, as it can assess skin damage before it is visible for the human eye.

In the other study, Dini and colleagues ${ }^{44}$ described a novel method and demonstrated differences in volatile emissions when loaded skin was compared with unloaded skin with solid phase micro extraction (SPME) - gas chromatography mass spectrometry (GC-MS) and an electronic nose. Although the findings of both studies are very 
promising, clinical prospective cohort studies need to be carried out to determine the predictive value of these new technologies.

\section{Cutaneous microbiome}

The skin is colonized by large amounts of bacteria, funghi and viruses. These microbes are essential in maintaining a healthy cutaneous environment by producing metabolites and stimulating the immune system. In general, four dominant phyla are colonizing the skin: Proteobacteria, Firmicutes, Bacteriodetes and Actinobacteria. However, the composition of the cutaneous microbiome depends on topographical location (Figure 1.1), skin type (dry, sebaceous, moist) ${ }^{45,46}$, and is highly variable between persons. The microbiome is very sensitive for changes in the cutaneous microenvironment and several factor have been associated with a changes in microbial composition such as Diabetes Mellitus, age, sex, washing behaviour or cosmetic use. In addition, disturbances in cutaneous microbiome occurs when skin becomes damaged and leads to recolonization of bacteria from the deeper skin layers ${ }^{47}$.

In 2007 the Human Microbiome Project ${ }^{48}$ was launched to characterize the microbiome and analyse its role in health and disease. Since then, multiple studies have used 16S RNA gene sequencing to detect whether there are differences in the composition of the cutaneous microbiome between healthy people in comparison with those suffering from a disease ${ }^{49}$. It appears that a shift in the cutaneous microbiome might play an important role in the pathophysiology of many skin diseases like acne ${ }^{50}$, atopic eczema ${ }^{51}$, and acute skin abscesses ${ }^{52}$. Identifying the microbiome and relate it to health and disease could lead to new diagnostic biomarkers and offers therapeutic potentials as well.

Several studies investigated the role of the cutaneous microbiome in chronic nonhealing pressure ulcer and wounds. However, it remains unclear if changes in the microbial composition of the unaffected skin increase the risk of developing pressure ulcers. When differences in the cutaneous microbiome are indeed associated with the development of pressure ulcers, it could possibly lead to new prognostic biomarkers (i.e. risk assessment), but may also lead to novel preventive interventions by modulating the cutaneous microbiome with antibiotics/ and or probiotics.

\section{Treatment of pressure ulcers}

One of the main principles in the treatment of pressure ulcers is to protect the skin for further damage. Minimizing the effect of pressure on skin by repositioning and pressure relieving support surfaces is essential ${ }^{53}$. Furthermore, elevation of the head of the bed should be discouraged to reduce the effect of shear on $\mathrm{skin}^{54}$. 


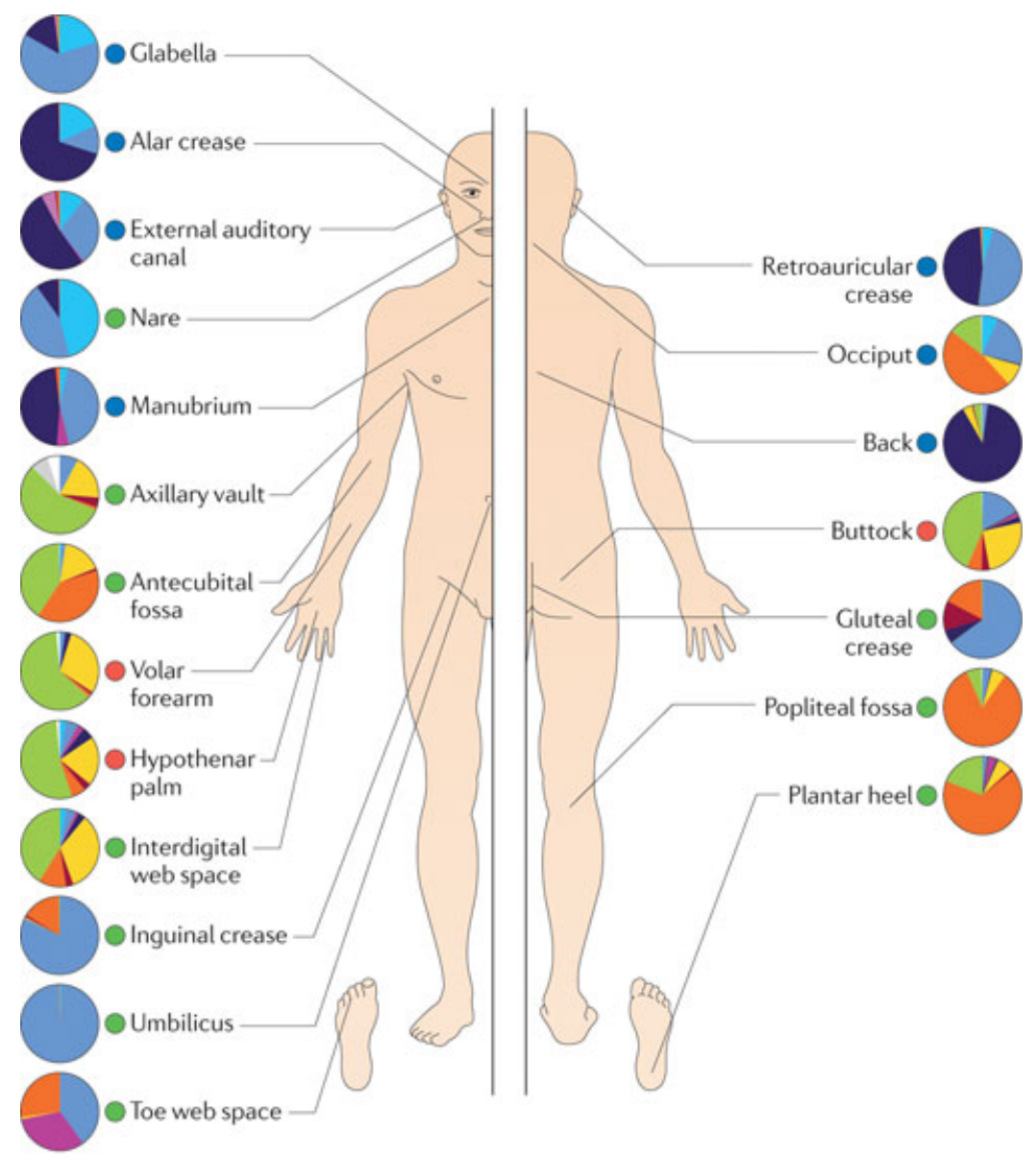

\begin{tabular}{|lll|}
\hline Actinobacteria & $\square$ Bacteroidetes & Proteobacteria \\
Corynebacteriaceae & Cyanobacteria & $\square$ Divisions contributing $<1 \%$ \\
Propionibacteriaceae & Firmicutes & $\square$ Unclassified \\
Micrococciaceae & Other Firmicutes & Sebaceous \\
Other Actinobacteria & Staphylococcaceae & Moist \\
& & Dry \\
& & \\
& & \\
& &
\end{tabular}

Nature Reviews | Microbiology

Figure 1.1 Topographical distribution of bacteria on skin sites From Grice et al. ${ }^{58}$ Nature Reviews 2011 
Another important component of pressure ulcer care is wound cleansing and debridement of necrotic tissue. Necrotic tissue decelerates the process to wound healing and provides an environment where bacteria can proliferate and form a biofilm, which eventually could lead to chronic non-healing wounds. In order to promote wound healing, a clean and viable wound bed is important to create an optimal environment for tissue regeneration. In addition, dietary intake and tissue perfusion should be optimized to provide enough oxygen and proteins to support the growth of granulation tissue and to promote pressure ulcer healing ${ }^{1,55}$.

Wound debridement can be performed by several techniques ${ }^{56}$.

Surgical debridement is the removal of necrotic tissue with the use of instruments such as scalpels or scissors.

Enzymatic debridement is the topical application of enzymes which are used to remove necrotic tissue.

Autolytic debridement is a common technique which is achieved by applying a wound dressing over the wound bed. Wound fluid and autolytic enzymes are captured underneath the dressing which digest necrotic tissue and promotes wound healing. The dressing should be removed every two or three days to clean the wound.

At last, surgical treatment of pressure ulcers can be considered as definitive treatment in pressure ulcers category 3 or 4 . Musculocutaneous, fasciocutaneous and perforator based flaps are all used for coverage of pressure ulcers. However, reconstructive surgery impose a major challenge for surgeons as it is associated with high recurrence and complications rates postoperatively ${ }^{57}$.

\section{Outline of the thesis}

The aim of this thesis was to investigate several new aspects in pressure ulcer prevention. All studies were performed at the Maastricht University Medical Centre (MUMC+) between 2012-2015.

We investigated if the cutaneous microbiome of unaffected skin is different in hospitalized patients with sacral pressure ulcers compared to hospitalized patients without pressure ulcers (Chapter 2).

In the next chapter (Chapter 3), on a shear model which was developed by the research team to apply a combined loading of pressure and shear on the volar aspect of the forearm of humans. This model was tested in ten healthy participants and several in vivo parameters: IL-1 $\alpha /$ TP, cutaneous blood cell flux and erythema index, were measured. These parameters were validated to measure the effects of shear in vivo. This newly developed model was used to investigate the effects of pressure and shear in patients with type 2 Diabetes Mellitus in comparison with healthy persons (Chapter 4) to regain more insight in the physiological response of skin on loading in patients with Diabetes Mellitus. 
Normally, prolonged immobilisation on a rigid spineboard may cause pressure ulcers. Therefore, we evaluated if lying on a soft-layered spineboard is better in preventing skin damage in healthy volunteers compared with lying on a rigid spineboard (Chapter 5).

In Chapter 6, the shear-model was used in to evaluate the shear reducing capacities of three different foam dressings in vivo.

Chapter 7 describes a randomized controlled trial to investigate if sacral pressure ulcers can be prevented with the use of a prophylactic sacral dressing. The trial was conducted in the MUMC+ between September 2012-September 2013. Patients were eligible to participate when they were at risk for the development of pressure ulcers according to the Braden or Prepurse scale. Outcomes were: Pressure ulcer incidence, VAS-scores for pain and discomfort, adverse events, device deficiencies and costs.

Finally, Chapter $\mathbf{8}$ is the thesis general discussion and provides implications for future research. 


\section{References}

1. Cereda E, Klersy C, Rondanelli M, Caccialanza R. Energy balance in patients with pressure ulcers: a systematic review and meta-analysis of observational studies. J Am Diet Assoc. 2011;111(12): 1868-1876.

2. Allman RM. Pressure ulcers among the elderly. N Engl J Med. 1989;320(13):850-853.

3. Coleman S, Gorecki C, Nelson EA, et al. Patient risk factors for pressure ulcer development: systematic review. Int J Nurs Stud. 2013;50(7):974-1003.

4. Beeckman D, Schoonhoven L, Fletcher J, et al. EPUAP classification system for pressure ulcers: European reliability study. J Adv Nurs. 2007;60(6):682-691.

5. Agrawal K, Chauhan N. Pressure ulcers: Back to the basics. Indian J Plast Surg. 2012;45(2):244-254.

6. Levine JM. Historical notes on pressure ulcers: the cure of Ambrose Pare. Decubitus. 1992;5(2): 23-24,26.

7. Levine JM. Historical perspective on pressure ulcers: the decubitus ominosus of Jean-Martin Charcot. J Am Geriatr Soc. 2005;53(7):1248-1251.

8. Reddy M, Gill SS, Rochon PA. Preventing pressure ulcers: a systematic review. JAMA. 2006;296(8): 974-984.

9. Sen CK, Gordillo GM, Roy S, et al. Human skin wounds: a major and snowballing threat to public health and the economy. Wound Repair Regen. 2009;17(6):763-771.

10. Reichel SM. Shearing force as a factor in decubitus ulcers in paraplegics. J Am Med Assoc. 1958; 166(7):762-763.

11. Dinsdale SM. Decubitus ulcers: role of pressure and friction in causation. Arch Phys Med Rehabil. 1974;55(4):147-152.

12. Oomens CW, Bader DL, Loerakker S, Baaijens F. Pressure induced deep tissue injury explained. Ann Biomed Eng.2015;43(2):297-305.

13. Gefen A, van Nierop B, Bader DL, Oomens CW. Strain-time cell-death threshold for skeletal muscle in a tissue-engineered model system for deep tissue injury. J Biomech. 2008;41(9):2003-2012.

14. Gawlitta D, Li W, Oomens CW, Baaijens FP, Bader DL, Bouten CV. The relative contributions of compression and hypoxia to development of muscle tissue damage: an in vitro study. Ann Biomed Eng. 2007;35(2):273-284.

15. Gefen A, Farid KJ, Shaywitz I. A review of deep tissue injury development, detection, and prevention: shear savvy. Ostomy Wound Manage. 2013;59(2):26-35.

16. Bliss MR. Hyperaemia. J Tissue Viability. 1998;8(4):4-13.

17. Fromy B, Lingueglia E, Sigaudo-Roussel D, Saumet JL, Lazdunski M. Asic3 is a neuronal mechanosensor for pressure-induced vasodilation that protects against pressure ulcers. Nat Med. 2012;18(8): 1205-1207.

18. Fromy B, Sigaudo-Roussel D, Gaubert-Dahan ML, et al. Aging-associated sensory neuropathy alters pressure-induced vasodilation in humans. J Invest Dermatol. 2010;130(3):849-855.

19. Fromy B, Abraham P, Bouvet C, Bouhanick B, Fressinaud P, Saumet JL. Early decrease of skin blood flow in response to locally applied pressure in diabetic subjects. Diabetes. 2002;51(4):1214-1217.

20. Jan YK, Shen S, Foreman RD, Ennis WJ. Skin blood flow response to locally applied mechanical and thermal stresses in the diabetic foot. Microvasc Res. 2013;89:40-46.

21. Oomens CW, Bader DL, Loerakker S, Baaijens F. Pressure induced deep tissue injury explained. Ann Biomed Eng. 2015;43(2):297-305.

22. Linder-Ganz E, Gefen A. The effects of pressure and shear on capillary closure in the microstructure of skeletal muscles. Ann Biomed Eng. 2007;35(12):2095-2107.

23. Goossens RH, Zegers R, Hoek van Dijke GA, Snijders CJ. Influence of shear on skin oxygen tension. Clin Physiol. 1994;14(1):111-118.

24. Vanderwee K, Grypdonck MH, De Bacquer D, Defloor T. Effectiveness of turning with unequal time intervals on the incidence of pressure ulcer lesions. J Adv Nurs. 2007;57(1):59-68.

25. Mclnnes E, Jammali-Blasi A, Bell-Syer SE, Dumville JC, Cullum N. Support surfaces for pressure ulcer prevention. The Cochrane database of systematic reviews. 2011(4):CD001735.

26. Mclnnes E, Jammali-Blasi A, Bell-Syer SE, Dumville JC, Middleton V, Cullum N. Support surfaces for pressure ulcer prevention. The Cochrane database of systematic reviews. 2015;9:CD001735. 
27. Demarre L, Van Lancker A, Van Hecke A, et al. The cost of prevention and treatment of pressure ulcers: A systematic review. Int J Nurs Stud. 2015;52(11):1754-1774.

28. Call E, Pedersen J, Bill B, et al. Enhancing pressure ulcer prevention using wound dressings: what are the modes of action? Int Wound J. 2015;12(4):408-413.

29. Ohura T, Takahashi M, Ohura N, Jr. Influence of external forces (pressure and shear force) on superficial layer and subcutis of porcine skin and effects of dressing materials: are dressing materials beneficial for reducing pressure and shear force in tissues? Wound Repair Regen. 2008;16(1):102-107.

30. Levy A, Frank MB, Gefen A. The biomechanical efficacy of dressings in preventing heel ulcers. J Tissue Viability. 2015;24(1):1-11.

31. Santamaria N, Gerdtz M, Sage S, et al. A randomised controlled trial of the effectiveness of soft silicone multi-layered foam dressings in the prevention of sacral and heel pressure ulcers in trauma and critically ill patients: the border trial. Int Wound J. 2015;12(3):302-308.

32. Brindle CT, Wegelin JA. Prophylactic dressing application to reduce pressure ulcer formation in cardiac surgery patients. J Wound Ostomy Continence Nurs. 2012;39(2):133-142.

33. Cubit K, McNally B, Lopez V. Taking the pressure off in the Emergency Department: evaluation of the prophylactic application of a low shear, soft silicon sacral dressing on high risk medical patients. Int Wound J. 2013;10(5):579-584.

34. Chaiken N. Reduction of sacral pressure ulcers in the intensive care unit using a silicone border foam dressing. J Wound Ostomy Continence Nurs. 2012;39(2):143-145.

35. Moore ZE, Webster J. Dressings and topical agents for preventing pressure ulcers. The Cochrane database of systematic reviews. 2013;8:CD009362.

36. Gefen A, Kottner J, Santamaria N. Clinical and biomechanical perspectives on pressure injury prevention research: The case of prophylactic dressings. Clin Biomechan. 2016;38:29-34.

37. Moore ZE, Cowman S. Risk assessment tools for the prevention of pressure ulcers. The Cochrane database of systematic reviews. 2014(2):CD006471.

38. Schoonhoven L, Haalboom JR, Bousema MT, et al. Prospective cohort study of routine use of risk assessment scales for prediction of pressure ulcers. BMJ. 2002;325(7368):797.

39. Pancorbo-Hidalgo PL, Garcia-Fernandez FP, Lopez-Medina IM, Alvarez-Nieto C. Risk assessment scales for pressure ulcer prevention: a systematic review. J Adv Nurs. 2006;54(1):94-110.

40. Kaczmarek A, Vandenabeele P, Krysko DV. Necroptosis: the release of damage-associated molecular patterns and its physiological relevance. Immunity. 2013;38(2):209-223.

41. Bronneberg D, Spiekstra SW, Cornelissen LH, et al. Cytokine and chemokine release upon prolonged mechanical loading of the epidermis. Exp Dermatol. 2007;16(7):567-573.

42. Cornelissen LH, Bronneberg D, Bader DL, Baaijens FP, Oomens CW. The transport profile of cytokines in epidermal equivalents subjected to mechanical loading. Ann Biomed Eng. 2009;37(5):1007-1018.

43. Swisher SL, Lin MC, Liao A, et al. Impedance sensing device enables early detection of pressure ulcers in vivo. Nat Commun. 2015;6:6575.

44. Dini F, Capuano R, Strand T, et al. Volatile emissions from compressed tissue. PloS One. 2013;8(7): e69271.

45. Grice EA. The skin microbiome: potential for novel diagnostic and therapeutic approaches to cutaneous disease. Semin Cutan Med Surg. 2014;33(2):98-103.

46. Grice EA, Kong $\mathrm{HH}$, Conlan $\mathrm{S}$, et al. Topographical and temporal diversity of the human skin microbiome. Science. 2009;324(5931):1190-1192.

47. Zeeuwen PL, Boekhorst J, van den Bogaard EH, et al. Microbiome dynamics of human epidermis following skin barrier disruption. Genome Biol. 2012;13(11):R101.

48. Turnbaugh PJ, Ley RE, Hamady M, Fraser-Liggett CM, Knight R, Gordon JI. The human microbiome project. Nature. 2007;449(7164):804-810.

49. Muszer M, Noszczynska M, Kasperkiewicz K, Skurnik M. Human microbiome: When a friend becomes an enemy. Arch Immunol Ther Exp. 2015;63(4):287-298.

50. Fitz-Gibbon S, Tomida S, Chiu BH, et al. Propionibacterium acnes strain populations in the human skin microbiome associated with acne. J Invest Dermatol. 2013;133(9):2152-2160.

51. Baviera G, Leoni MC, Capra L, et al. Microbiota in healthy skin and in atopic eczema. BioMed Res Int. 2014;2014:436921.

52. Horton JM, Gao Z, Sullivan DM, Shopsin B, Perez-Perez GI, Blaser MJ. The cutaneous microbiome in outpatients presenting with acute skin abscesses. J Infect Dis. 2015;211(12):1895-1904. 
53. Gould L, Stuntz M, Giovannelli M, et al. Wound Healing Society 2015 update on guidelines for pressure ulcers. Wound Repair Regen. 2016;24(1):145-162.

54. Mimura M, Ohura T, Takahashi M, Kajiwara R, Ohura N, Jr. Mechanism leading to the development of pressure ulcers based on shear force and pressures during a bed operation: influence of body types, body positions, and knee positions. Wound Repair Regen. 2009;17(6):789-796.

55. Dorner B, Posthauer ME, Thomas D, National Pressure Ulcer Advisory P. The role of nutrition in pressure ulcer prevention and treatment: National Pressure Ulcer Advisory Panel white paper. Adv Skin Wound Care. 2009;22(5):212-221.

56. McCallon SK, Weir D, Lantis JC, 2nd. Optimizing wound bed preparation with collagenase enzymatic debridement. J Am Coll Clin Wound Spec. 2014;6(1-2):14-23.

57. Sameem M, Au M, Wood T, Farrokhyar F, Mahoney J. A systematic review of complication and recurrence rates of musculocutaneous, fasciocutaneous, and perforator-based flaps for treatment of pressure sores. Plast Reconstr Surg. 2012;130(1):67e-77e.

58. Grice EA, Segre JA. The skin microbiome. Nat Rev Microbiol. 2011;9(4):244-253. 



\section{Chapter 2}

The cutaneous microbiome in hospitalized patients with pressure ulcers

L.A. de Wert, S.S. Rensen' Z. Soons, M. Poeze, N.D. Bouvy, J. Penders Submitted 


\section{Abstract}

\section{Background}

This study investigated whether there are differences in the composition of the cutaneous microbiome of the unaffected skin between patients with pressure ulcers compared with those without pressure ulcers.

\section{Results}

The cutaneous microbiome of the unaffected skin of 15 patients with sacral pressure ulcers compared to 15 patients without pressure ulcers was analysed.

It demonstrated that the inter-individual variation in skin microbiota of patients with pressure ulcers was significantly higher $(\mathrm{P}=0.01)$. The abundance of 23 species was significantly different with Staphylococcus Aureus and unclassified Enterococcus the most abundant species in patients with pressure ulcers.

Using Random Forrest prediction models showed that eight species predicted pressure ulcer status correctly in $81 \%$ of the patient. A subset of four species gave the strongest interaction. The presence of unclassified Enterocuccus had the highest impact on pressure ulcer status.

\section{Conclusions}

This study is the first to demonstrate that the cutaneous microbiome differs in patients with pressure ulcers. A multivariate approach leaded to an improved classification and was more robust and more prognostic than a single species. In the future, the cutaneous microbiome could possibly be used as a biomarker to predict patients at risk and could lead to targeted preventive interventions. 


\section{Introduction}

Pressure ulcers, or decubitus, are an important clinical problem, especially in the elderly, disabled or those who are bound to a bed or wheelchair. Yearly, over 2.5 million hospitalized patients are treated in the United States for pressure ulcers with estimated costs of 11.0 billion U.S. Dollars ${ }^{1,2}$. Pressure ulcers develop as a result of prolonged mechanical loading on the skin over a bony prominence, mostly at the sacral area. Several internal factors can further contribute to the development of pressure ulcers. For example, increasing age; unconsciousness; urinary and/ or bowel incontinence; poor nutritional status; Diabetes Mellitus; paralysis and cardiovascular diseases have been associated with an increased risk of developing pressure ulcers ${ }^{3}$. The human skin harbours a large number of microorganisms, the so called microbiota including bacteria and fungi. A well- balanced cutaneous microbiota is essential in maintaining a healthy skin environment. Indeed, many skin diseases are associated with changes in microbiota composition. For example, an increase in relative Staphylococcus aureus abundance plays an important role in the pathophysiology of atopic dermatitis flares, while certain strains of Propionibacterium acnes contributes to the development of Acnes Vulgaris ${ }^{4}$. In wounds, bacteria impair healing by forming a biofilm, which eventually, may lead to chronic non-healing wounds ${ }^{5,6}$.

In view of the impact of skin bacteria on the development of skin disorders as highlighted above, cutaneous microbiota differences may significantly contribute to the risk of developing pressure ulcers. Such potential differences may offer a new way to identify patients at increased risk for pressure ulcers and can lead to new preventive measures based on modulation of the microbiota as well ${ }^{7}$.

Therefore, the aim of this study was to examine the cutaneous microbiome of the unaffected skin of hospitalized patients with and without sacral pressure ulcers.

\section{Methods}

\section{Participants}

We conducted a single centre, cross-sectional, case-control study at the Maastricht University Medical Centre (MUMC+). The study was approved by the medical ethics committee of the MUMC+ and written informed consent was obtained from all the study participants. The study was conducted in compliance with ethical rules for human experimentation that are stated in the Declaration of Helsinki. A total of thirty patients were able to participate at this study; 15 patients with a category $\geq 2$ sacral pressure ulcer (decubitus group, DC) and 15 patients with no (pre-)existing pressure ulcers (control group, NoDC). All patients had to be admitted to one of the wards of the MUMC+. Exclusion criteria were: age $<18$ years, mentally disabled patients/ 
unable to give informed consent, any skin diseases/ wounds/ signs of infection at the skin of vertebrae level L3.

\section{Sample collection}

To study the cutaneous microbiome of the unaffected skin, skin samples were obtained using Copan FloqSwabs ${ }^{\mathrm{TM}}$ (Copan Flock Technologies, Brescia, Italy) under sterile conditions. First, the skin of vertebrae level L3 was inspected for any signs of infection (erythema), skin diseases or wounds. When the skin was unaffected, an area of $4 \mathrm{~cm}^{2}$ was marked with a skin marker. The swab was pre-moistened with Reduced Transport Fluid (RTF) buffer ${ }^{26}$. Then, the skin was stretched and swabbed 50 times back and forth applying firm pressure. After swabbing, the tips were placed in a vial containing $50 \mu$ l of RTF buffer and immediately placed on dry ice before it was stored in a $-80^{\circ} \mathrm{C}$ freezer until further processing. All samples were collected between 01.00-03.00 PM. To prevent bias due to variation in sample collection, all skin samples were obtained by one single researcher.

Subsequently, sex, age, length, weight, medical diagnosis, co- morbidities, antibiotics use, pressure ulcer category and pressure ulcer treatment were recorded for each patient.

\section{DNA isolation}

After adding $180 \mu$ lysis buffer (20 mM Tris pH8, 2 mM EDTA pH8, 1.2\% Triton X-100) with lysozyme $(20 \mathrm{mg} / \mathrm{ml})$, swabs were incubated for $30 \mathrm{~min}$ at $37^{\circ} \mathrm{C}$ and subsequently mixed by vortexing. After adding $200 \mu$ l Buffer $\mathrm{AL}$ and $25 \mu \mathrm{l}$ Proteinase $\mathrm{K}$ from the QIAamp DNA mini kit (Qiagen, Hilden, Germany) and mixing by vortexing, samples were incubate overnight at $56^{\circ} \mathrm{C}$. The next day, $200 \mu$ of ethanol (96-100\%) was added and upon mixing by vortexing the complete lysate. Subsequent steps were conducted according to the protocol of the QIAamp DNA mini kit as per manufacturer's instructions, except that the DNA was eluated in a final volume of $100 \mu \mathrm{l}$.

\section{Sequencing}

Amplicon libraries and sequencing was performed according to previously published protocols ${ }^{27}$. Briefly, the V4 region of the 16S rRNA gene was PCR amplified from each DNA sample in triplicate using the 515f/806r primer pair ${ }^{27}$. Pooled amplicons from the triplicate reactions were purified using AMPure XP purification (Agencourt, Massachusetts, USA) according to the manufacturer's instructions and eluted in $25 \mu \mathrm{l}$ 1× low TE (10 mM Tris- $\mathrm{HCl}, 0.1 \mathrm{mM}$ EDTA, $\mathrm{pH}$ 8.0) and subsequently quantified by Quant-iT PicoGreen dsDNA reagent kit (Invitrogen, New York, USA) using a Victor3 Multilabel Counter (Perkin Elmer, Waltham, USA). Amplicons were mixed in equimolar 
concentrations to ensure equal representation of each sample and sequenced on an Illumina MiSeq instrument.

\section{Data analysis and statistics}

The V4 16S rDNA bacterial sequences generated in the present study were deposited in QIITA archive (accession number: 11145). Filtering, denoising, removing of chimeric sequences and clustering of sequences in Operational Taxonomic Units (OTUs) at $97 \%$ similarity was conducted using the LotuS (version 1.39) pipeline ${ }^{28}$. First, sequences with an average quality below 27, read length below 170 bases, one or more ambiguous bases, or containing homopolymer stretches of over 8 bases were discarded for further analysis. Retained sequences were chimera filtered and clustered into OTUs with UPARSE ${ }^{29}$. Taxonomic annotation of OTUs was derived from RDP naïve bayes classifier annotations (minimum acceptance confidence set at 0.8$)^{30}$. Singletons and OTUs with less than 10 reads were removed. QIIME version $1.8 .1^{31}$ and $\mathrm{R}$ version 3.1.3 were used to conduct downstream analyses.

\section{Assessing differences on a species level}

To examine potential differences in the relative abundance of bacterial species between pressure ulcer patients (DC) and control patients (NoDC), all OTUs that were taxonomically assigned to the same species were combined.

For all subsequent analyses, we normalized the count-table using variant stabilization by the R-package DESeq $2^{32}$. The number of sequence reads ranged from 29,073 to 258,211 species counts in one sample. We used size factor correction to account for these differences in sequencing depth between the samples. DESeq2 was also applied to obtain dispersion estimates and to test for differential abundance of each species between pressure ulcer patients (DC) and control patients (NoDC) using default parameters. Results are reported as log2 fold changes and associated adjusted pvalues (BH-correction).

Dendrograms were obtained by hierarchical clustering using Ward's method where Pearson's correlation was used as the distance measure.

\section{Microbial alpha and beta diversity}

The following metrics of species richness and diversity within communities (alphadiversity) were determined: Observed OTUs (observed richness), Chao1 index (estimated richness), Shannon index (estimated diversity) and Good's coverage. Normality was tested with the D'agostino\& Pearson omnibus test. The Mann Whitney $U$ test was used to compare the alpha diversity metrics among the patients in the DC group to the patients in the NoDC group.

The dissimilarity in the microbial community composition (beta-diversity) between each pair of skin samples was assessed using the Bray-Curtis and (un)weighted UniFrac distance ${ }^{8}$ and Bray-Curtis dissimilarity (BC). Clustering of samples was 
visualized using Principal Coordinate analysis (PCOA). Nonparametric two- sample ttests with Bonferroni correction based upon 1000 Monte Carlo permutations were used to compare the beta-diversity within pressure ulcer patients (average pairwise distance between all samples in the DC group) to the beta diversity within the control patients (NoDC) (average pairwise distance between all samples in the NoDC group) and the between group beta-diversity (average pairwise distance between samples of each patient in the DC group and each NoDC group patient).

\section{Machine learning}

Random forests are an effective approach for analyzing and interpreting highdimensional data. The method correlates species abundance or clinical data with pressure ulcer status and introduces a novel bivariate node-splitting. We used the $R$ package randomForestSRC [Ishwaran et al., 2010] and the Boruta algorithm for feature selection. The bootstrapped feature selection was repeated 1000 times with differing random seeds. A number of trees of 500 was found to produce a stable error rate.

\section{Results}

\section{Study population}

Thirty patients were included in this study between July 2015 and August 2015, 15 patients with pressure ulcers (DC group) and 15 control patients (NoDC group). All patients were bedridden and admitted to one of the wards of MUMC+. Patients were matched for age, sex, BMI, Diabetes Mellitus, antibiotics use and medical diagnosis. Baseline characteristics are presented in Table 2.1.

\section{Sequencing and taxonomic composition}

A total of 5,021,668 paired-end reads were generated. After trimming, quality filtering, removal of potential chimeric reads, 3,707,991 sequences were retained for downstream analysis and clustered into 2,169 operational taxonomic units (OTUs). Samples from three control participants failed during sequencing, because of low bacterial DNA yield, and were discarded for subsequent analysis. The number of sequences for the remaining samples ranged from 48,952 to 259,953 (median $117,362)$. 
Table 2.1 Baseline characteristics of included participants $(n=30)^{*}$

\begin{tabular}{lcc}
\hline & DC group $(\mathbf{n}=\mathbf{1 5})$ & NoDC $(\mathbf{n}=\mathbf{1 5})$ \\
\hline Age- years & $72.8 \pm 8.8$ & $73.2 \pm 5.5$ \\
Male sex & $9(60.0)$ & $9(60.0)$ \\
Length- centimetres & $172.4 \pm 9.2$ & $169.9 \pm 13.5$ \\
Weight- kilogram & $78.8 \pm 20.0$ & $76.0 \pm 12.6$ \\
BMl- kg/cm & $26.2 \pm 5.5$ & $26.7 \pm 4.0$ \\
Antibiotics use & $9(60.0)$ & $8(53.3)$ \\
Diagnosis of hospital admission & & \\
$\quad$ Orthopaedic & $7(46.7)$ & $6(40.0)$ \\
Gastro-intestinal & $4(26.7)$ & $6(40.0)$ \\
Cardiovascular & $3(20.0)$ & $2(13.3)$ \\
$\quad$ Respiratory & $1(6.7)$ & $1(6.7)$ \\
Comorbidities & & $3(20.0)$ \\
$\quad$ Diabetes Mellitus & $3(20.0)$ & \\
\hline
\end{tabular}

Data are presented as mean \pm SD or number (\%). NS= Not significant. *This number includes the samples of three patients in the control group which failed during sequencing, because of low bacterial DNA yield and were removed from further analysis.

\section{Taxonomic composition}

In both patients groups, Firmicutes, Proteobacteria and Actinobacteria were the most abundant bacterial phyla, whereas at the genus level Staphylococcus spp. and Corynebacterium spp. predominated (Figure 2.1A,B).

\section{Microbial richness and diversity are not altered in patients with sacral pressure ulcers}

The DC and NoDC groups did not show a significant difference with respect to average number of observed OTUs in patients of the DC group (245.0, (168.0-370.0)) and NoDC group (299.5 (163.0-478.0)) as well as the estimated richness of the DC groups (Chao1, 299.6 (195.1-1112) and No DC group (Chao1, 287.8 (204.0-504.3), did not differ significantly (Figure $2.2 A, B$ ). The Good's estimator suggested $>99.95 \%$ coverage for all samples included in the present study, indicating that only an additional five OTUs would be found if the sequencing depth was increased with another 1,000 reads. Also, microbial diversity as estimated by the Shannon diversity was not statistically significantly different in skin swabs between DC and NoDC group, although a larger variation in microbial diversity was observed in the DC group (Figure 2.2C). Altogether these results indicate that the microbial richness and evenness are not affected in patients with sacral pressure ulcers. 

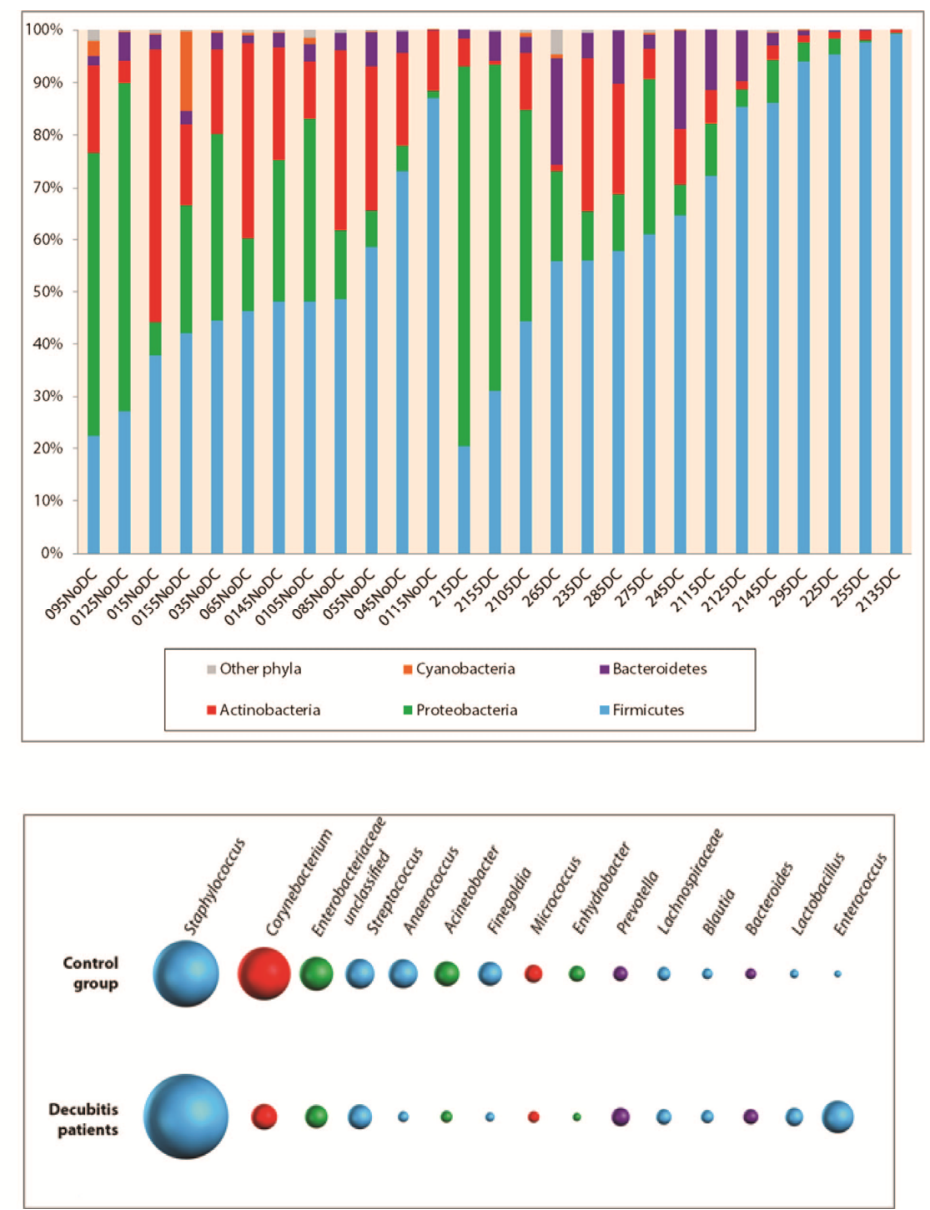

Figure 2.1 Relative abundance of the most predominant bacterial phyla in the control (NoDC) and decubitus group (DC) patients. B. Average proportion of the 15 most abundant bacterial genera in the control group (NoDC) and decubitus group (DC) patients.

A

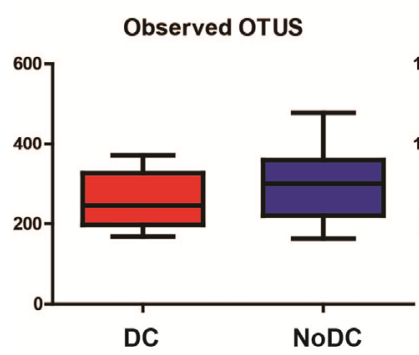

B

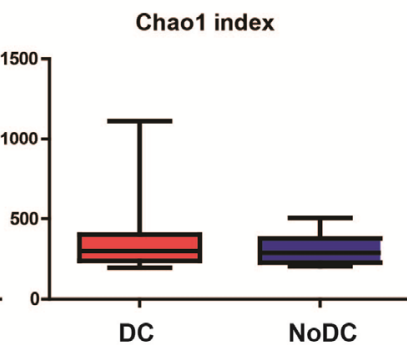

C

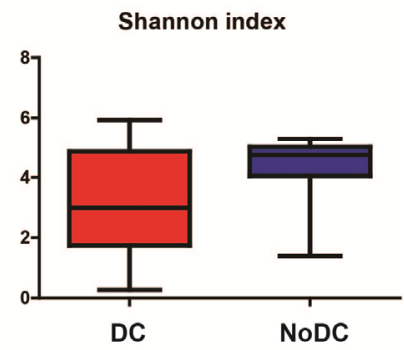

Figure 2.2 Box and Whisker plots of the Alpha diversity metrics. No statistically significant differences were observed between the DC and NoDC group patients (tested by Mann Whitney $\mathrm{U}$ test $(\mathrm{P}>0.05))$. 


\section{The skin microbial community structure is related to disease status}

Next, we assessed the dissimilarity in the microbial composition (beta-diversity) of the skin using the Bray-Curtis and the weighted Unifrac distances. Unifrac distances are based on the fraction of branch length shared between two communities within a phylogenetic tree constructed form the $16 \mathrm{~S}$ rRna gene sequences from all communities being compared. A relatively small UniFrac distance implies that two communities are compositionally similar, harbouring lineages sharing a common evolutionary history ${ }^{8}$.

Visualization of Bray-Curtis and weighted Unifrac dissimilarities using PCoA, indicated that many, but not all patients in the DC group clustered apart from the NoDC group patients (Figure 2.3A,C). This implies that the skin microbial communities in part of the patients with sacral pressure ulcers are structurally different from the microbial communities in patients without pressure ulcers.

Moreover, the dissimilarity in the microbial community structures in skin swabs within the DC group was on average larger than the dissimilarity within the NoDC group. This indicates that the inter-individual variation in the skin microbiota of patients without sacral pressure ulcers is significantly smaller as compared to the inter-individual variation in the cutaneous microbiome of patients with sacral pressure ulcers (Figure 2.3B. $P=0.01$ for Bray-Curtis and Figure 2.3D. $P=0.01$ for weighted Unifrac).

\section{Species abundance}

Hierarchical clustering based upon the species level relative abundance revealed distinct clusters for half of the patients with and without pressure ulcers (Figure 2.4A). A few patients in the DC group were closer to disease free patients of the NoDC group based on the overall species abundancies. We next focussed on the species that distinguish patients with and without pressure ulcers (DC and NoDC group). The abundance of 23 species was significantly different, ranging from a 184-fold more abundant presence (log2 fold change of 7.5 ) to a 34 fold less abundant presence (log2 fold-change of 5.1) in the DC group. Staphylococcus Aureus and unclassified Enterococcus were the most abundant species in the DC group. (Figure 2.4B,C).

\section{Random forest}

To further test the potential clinical relevance of bacterial species and clinical factors in pressure ulcers, we carried out a Random Forest (RF) analysis combining the species abundances, resp. clinical data. We found that the clinical data in Table 2.1, such as sex, antibiotics use, and BMI, were not significantly related with pressure ulcer status. Supplementary Figure S2.1. Shows that none of the clinical factors shows a higher correlation to pressure ulcer outcome than the best random predictor. In addition, when all clinical data were taken together, the observed error rate (41\%) is not significantly better either than a random predictor in a Fisher test (50\%). 
A

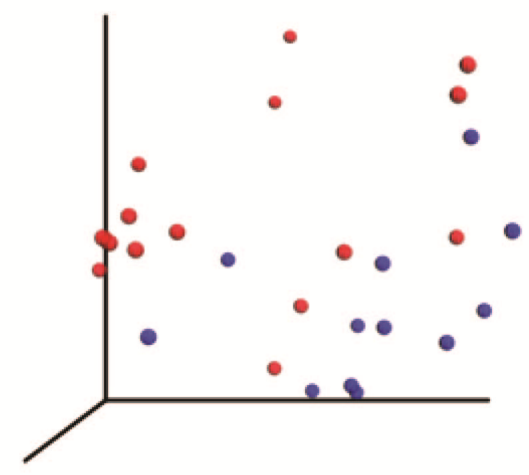

B
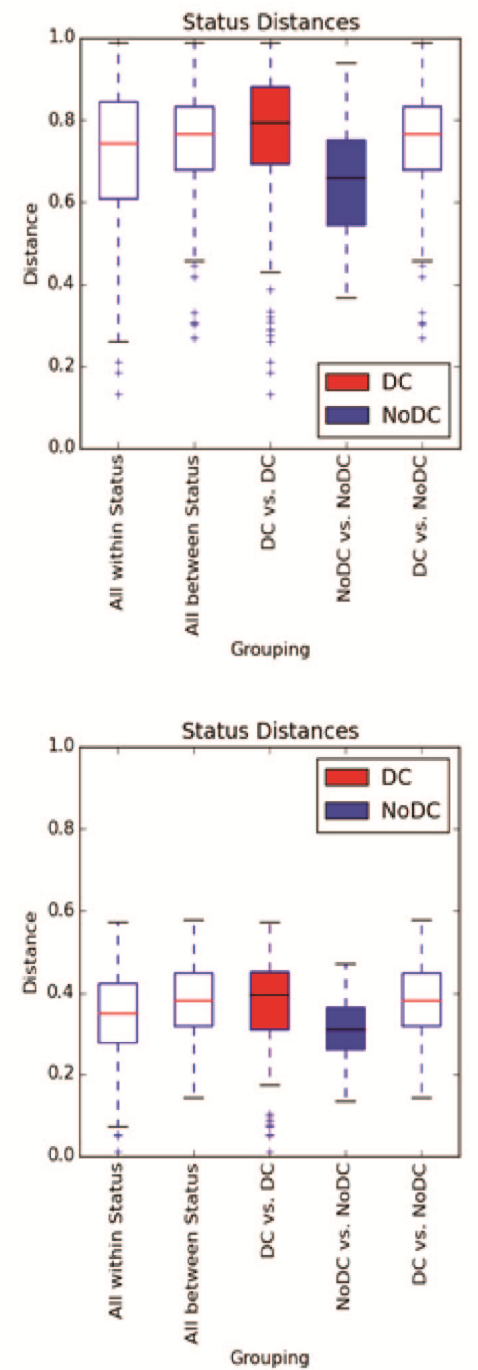

Figure 2.3 Taxonomy at phylum and genus level for patients with and without sacral pressure ulcers. A. Principal Coordinate Analyses (PCOAs) based on Bray-Curtis distance coloured according to disease status (DC group patients in red and NoDC group patients in blue). Variation explained by the principal coordinates: PC1 (29.1\%); PC2 (12.7\%); PC3 (8.64\%). B. Box and Whisker plots of the within and between group Bray-Curtis distances. C. Principal Coordinate Analyses (PCoAs) based on weighted Unifrac distance coloured according to disease status (decubitus group patients in red and control group patients in blue). Variation explained by the principal coordinates: PC1 (28.8\%); PC2 (18.5\%); PC3 (16.8\%). D. Box and Whisker plots of the within and between group weighted Unifrac distances. 
A

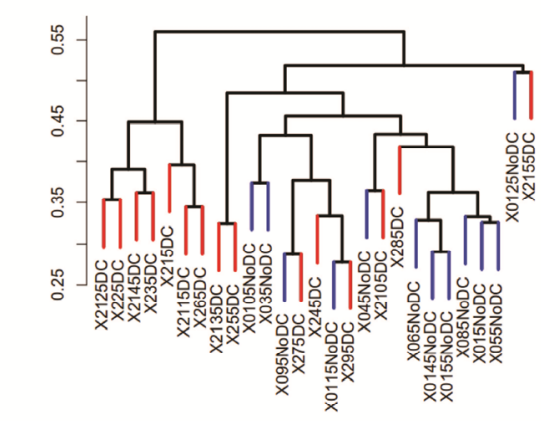

C
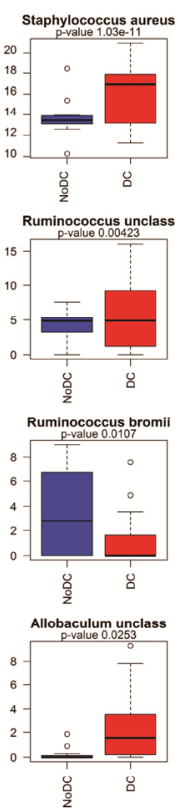
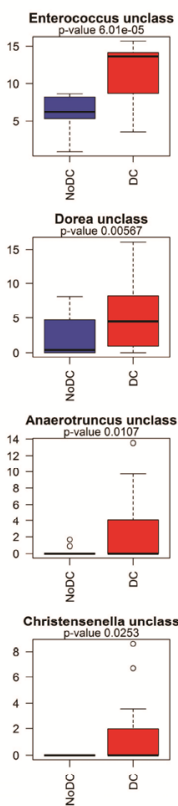

B
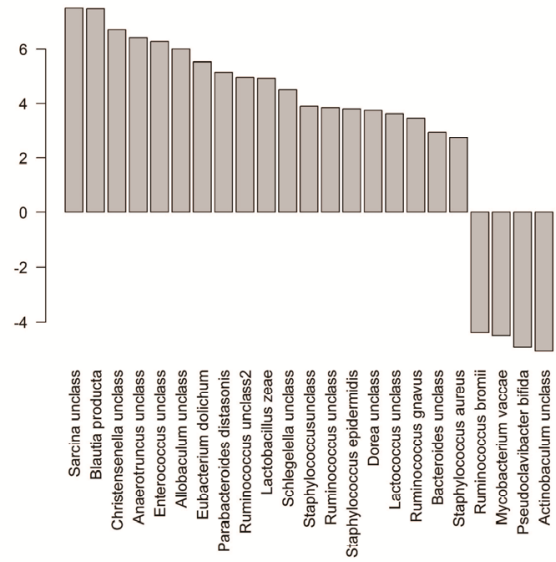
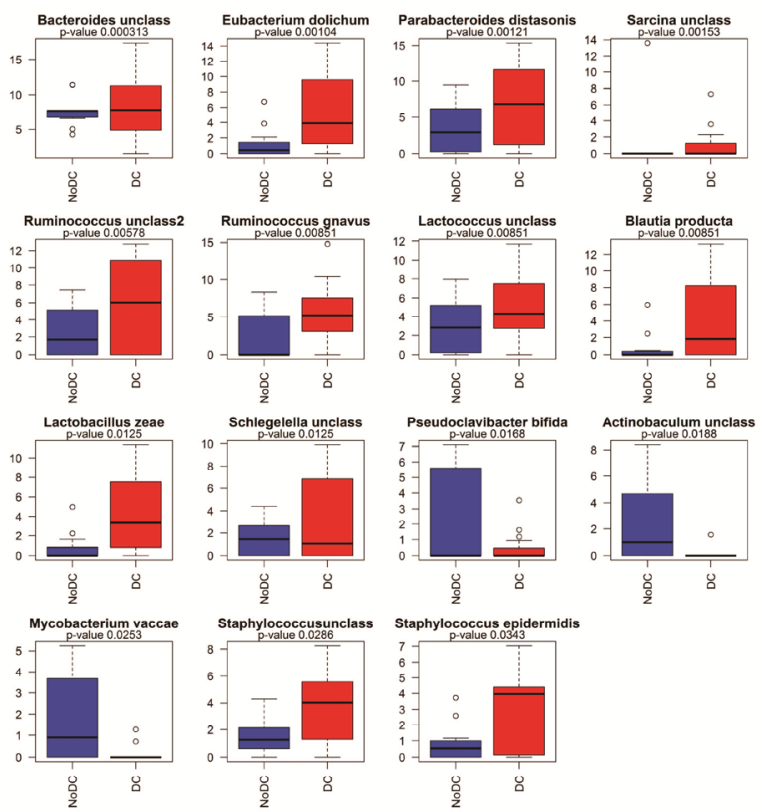

Figure 2.4 Differential species abundancies between DC and NoDC group patients. A. Cluster diagram. B. Log2 fold changes of the species with significantly different abundance in DC vs. NoDC group patients. C. Boxplots of $\log 2$ abundancies of these 23 species

Classification based on species abundance, however, showed that eight species are significantly related to pressure ulcer status in the Boruta algorithm (Figure 2.5A). These eight species together classified pressure ulcer status correctly in $81 \%$ of the patients (error rate of 19\%) (Figure 2.5C). Figure 5D shows the expected probability of having a pressure ulcer as function of species abundance, adjusted for all other species abundances. High abundance of for instance, unclassified Actinobaculum is 
negatively associated with a higher probability of having a pressure ulcer, whereas the abundance of unclassified Enterocuccus is positively associated with higher probability of having a pressure ulcer.

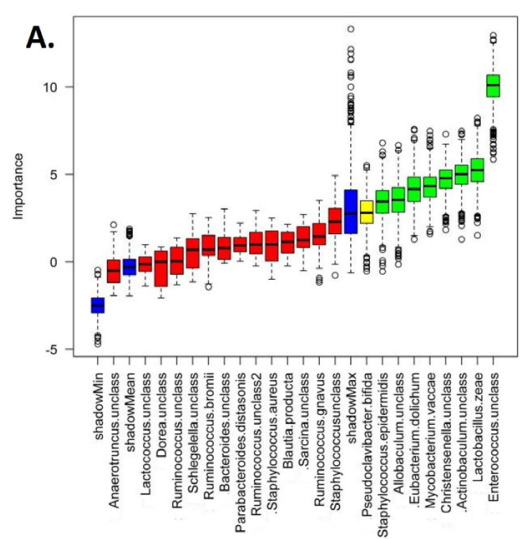

c.

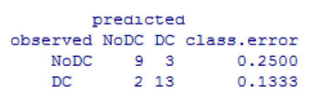

Overall error rate: $18.52 \%$

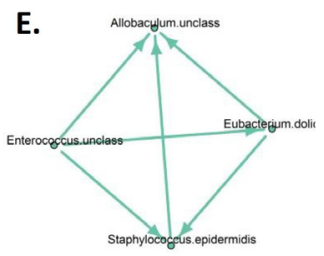

B. all

Enterococcus.unclass

Lactobacillus.zeae

Eubacterium.dolichum

Mycobacterium.vaccae

Actinobaculum.unclass

Staphylococcus.epidermidis

Allobaculum.unclass

Christensenella.unclass

NoDC

Enterococcus.unclass

Lactobacillus.zeae

Eubacterium.dolichum

Mycobacterium.vaccae

Actinobaculum unclass

Staphylococcus.epidermidis

Allobaculum.unclass

Christensenella.unclass

DC

Enterococcus.unclass

Lactobacillus.zeae

Eubacterium.dolichum

Mycobacterium.vaccae

Actinobaculum.unclass

Staphylococcus.epidermidis

Allobaculum.unclass

Christensenella.unclass

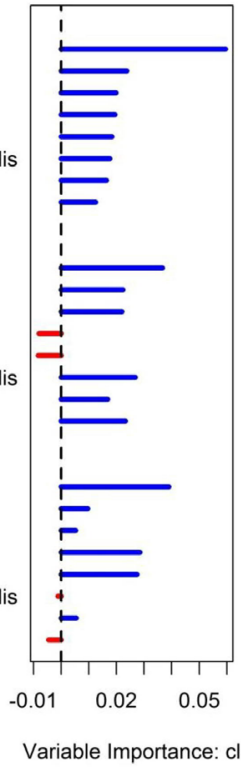

D.

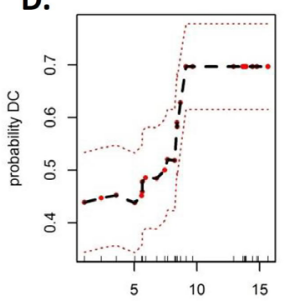

Enterococcus.unclass

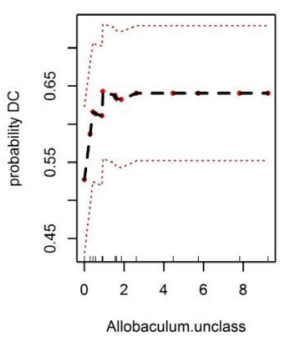

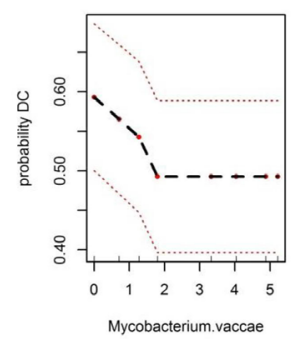

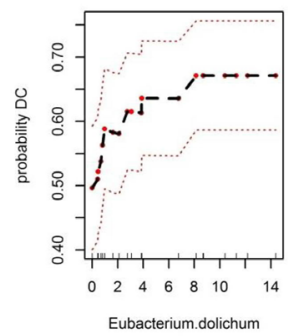

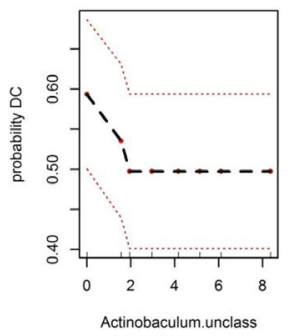

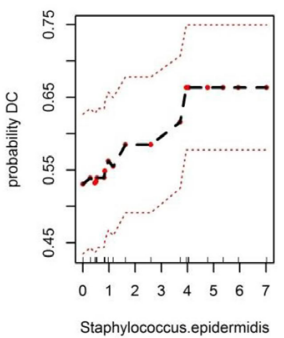

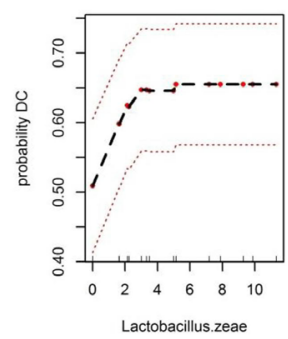

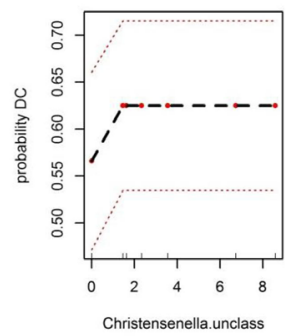

Figure 2.5 Random Forests with Boruta feature selection using species abundance data. A. The importance of each feature across 1000 repeats is shown compared with the worst, best, and mean randomly generated features. B. Variable importance in the random forest. 
C. Confusion matrix. D. Effect of species abundance on expected pressure ulcer status in the random forest model. Values on the vertical axis represent the expected probability of a pressure ulcer for a given species, after adjusting for all other predictors. Dashed red lines represent \pm 2 standard error. Expected probability based on random classification is 0.44 . $\mathbf{E}$. Interaction plot.

Interestingly, a subset of four species giving the strongest interactions in an interaction plot (Figure 2.5E), unclassified Enterococcus, unclassified Allobaculum, Eubacterium dolichum and Staphylocuccus epidermidis, correctly classify pressure ulcer status in $74 \%$ of the patients (error rate of $26 \%$ ). Species tending to split close to the root node have a strong effect on classification, whereas species that split further down the tree have less impact on disease status. More specifically, the presence of unclassified Enterocuccus has the highest impact on pressure ulcer status. Since unclassified Enterocuccus, unclassified Allobaculum, Eubacterium dolichum and Staphylocuccus epidermidis are highly abundant in different patients, a combination of these four species contains additive information leading to better classification of pressure ulcer status.

\section{Discussion}

The cutaneous microbiome plays an important role in providing a healthy environment of the skin, and microbiome alterations influence host-microbe interactions leading to changes in host metabolism and immunity ${ }^{9}$. Therefore, maintaining a well-balanced cutaneous microbiome is essential in preventing the development of skin diseases. Although the development of pressure ulcers is mainly related to prolonged mechanical loading in the form of pressure and shear forces, local changes in the skin microclimate have been shown to be a marked risk factor for the development of pressure ulcers in earlier studies ${ }^{10-12}$. In addition, changes in environmental factors influences the skin microenvironment and leads to differences in the composition of the cutaneous microbiome ${ }^{13}$.

To our knowledge, this study is the first to investigate whether there are differences in the cutaneous microbiome of the unaffected skin between hospitalized patients with pressure ulcers compared with those without pressure ulcers. It demonstrates that the microbiota of the unaffected skin in patients with sacral pressure ulcers differs from those without pressure ulcers. Although microbial diversity and richness were not different between patients with or without sacral pressure ulcers, there was an increase in inter-individual variation of the cutaneous microbiome in patients with sacral pressure ulcers. In addition, the abundance of 23 bacterial species on the skin were significantly different in patients with sacral pressure ulcers.

It is particularly promising that we were able to identify eight species that predict pressure ulcer status with RF prediction models. A high abundance of one of defined 
six species was associated with a higher probability of sacral pressure ulcers, suggesting that patients with a higher abundance of one of these species might already be at higher risk for developing sacral pressure ulcers. Combining information on the abundance of several species further improved the classification of the presence of a pressure ulcer. Therefore, predicting pressure ulcer risk based on a multivariable approach with microbial species as biomarkers provides a potential novel opportunity to pressure ulcer risk assessment in hospitalized patients in the future.

It is interesting to note that unclassified Enterococcus and Eubacterium dolichium, both commensal gut bacteria, contribute most to the RF prediction model. Furthermore, a higher abundance of several other gut bacteria Christensenella, Lactobacillus zeae and unclassified Allobaculum was also associated with a higher probability of pressure ulcer status. In hospitalized patients, up to $17 \%$ have some form of faecal incontinence and it is a daily challenge for healthcare workers to prevent faecal incontinence in patients ${ }^{14}$. Although faecal incontinence is associated with an increased pressure ulcer risk ${ }^{15,16}$, there are also studies with contradictory results $^{17,18}$. However, based on the results of the present study, it seems beneficial to protect the skin against faecal incontinence to prevent the development of pressure ulcers. This could lead to the development of targeted preventive interventions to decrease the abundance of certain gut bacteria on the skin. For example, topical agents/ wound dressings containing antibiotics or probiotics could be implemented in addition to already existing pressure ulcer preventive strategies.

On the other hand, a higher abundance of four species was found in patients without pressure ulcers, where two species in particular, unclassified Actinobaculum and Myobacterium vaccae, were predictive for a pressure ulcer free status in the RF prediction models. Interestingly, Keshavarz Valian and colleagues ${ }^{19}$ demonstrated that the administration of a low dose of Myobacterium vaccae protected mice against the development of skin ulcera caused by a parasitic infection with Leishmania major. Therefore, there might be a role of specific types of bacteria in protecting the skin against the development of wounds such as pressure ulcers in humans as well.

The cutaneous microbiome is known to be influenced by several factors such as BMI, Diabetes mellitus ${ }^{20}$, antibiotic use ${ }^{21}$, sex $^{22}$ and age ${ }^{23}$. The results of our study did not reveal any relation of these clinical factors with pressure ulcers. This supports that differences in outcomes in the current study are not likely to be caused by confounding factors. Other measures to prevent external influences on outcomes included: sampling by one member of the research team; sampling in nearly the same weather conditions (July-August); and at almost the same time every day (01.00-03.00 PM).

Some comments needs to be placed with respect to the interpretation of our results. First, we focussed on sacral pressure ulcers while pressure ulcers can develop at other skin areas (e.g. the heels, elbows, hips) as well. This might limit the generalizability to patients who developed pressure ulcers at different sites, because the composition of 
the cutaneous microbiome depends on the type of microenvironment (dry, moist, sebaceous). ${ }^{13}$ We chose to focus on sacral pressure ulcers, because those are most common in hospitalized patients ${ }^{24,25}$, and are more difficult to prevent compared to pressure ulcers at other anatomical locations.

In addition, we chose to obtain skin swabs from the skin of vertebrae level L3and not to obtain samples from the sacral area itself, because we wanted to obtain skin samples from the unaffected skin and not from the pressure ulcers itself. Although we did not obtain skin swabs from the sacral area, it is thought that the type of cutaneous microenvironment of vertebrae level L3 is comparable with those of the sacral area ${ }^{13}$. Second, this study is limited by the relatively small sample size, but also its crosssectional design. Despite the small sample size, statistically significant differences were found between the two groups. However, it is impossible to determine if these differences in cutaneous microbiome caused changes in skin physiology that eventually promoted the development of pressure ulcers, or whether the microbial shifts were a consequence of alterations in skin physiology/ microenvironment as a result of prolonged mechanical loading. Future longitudinal studies should assess whether the cutaneous microbiome is already disturbed prior the manifestations of pressure ulcers.

In conclusion, this study is the first to demonstrate that the cutaneous microbiome of the unaffected skin differs in patients with sacral pressure ulcers from those without pressure ulcers. In the future, this could lead to better prediction of patients at risk for pressure ulcers with the use of the cutaneous microbiome as a biomarker. Furthermore, it could lead to improved preventive interventions by modulating the cutaneous microbiome in a way that is beneficial for the host. 


\section{References}

1. Sen CK, Gordillo GM, Roy S, et al. Human skin wounds: a major and snowballing threat to public health and the economy. Wound Repair Regen. 2009;17(6):763-771.

2. Brem H, Maggi J, Nierman D, et al. High cost of stage IV pressure ulcers. Am J Surg. 2010;200(4): 473-477.

3. Coleman S, Gorecki C, Nelson EA, et al. Patient risk factors for pressure ulcer development: systematic review. Int J Nurs Stud. 2013;50(7):974-1003.

4. Findley K, Grice EA. The skin microbiome: a focus on pathogens and their association with skin disease. PLoS Pathog. 2014;10(10):e1004436.

5. Costerton JW, Stewart PS, Greenberg EP. Bacterial biofilms: a common cause of persistent infections. Science. 1999;284(5418):1318-1322.

6. Wolcott RD, Hanson JD, Rees EJ, et al. Analysis of the chronic wound microbiota of 2,963 patients by 16S rDNA pyrosequencing. Wound Repair Regen. Oct 142015.

7. Turnbaugh PJ, Ley RE, Hamady M, Fraser-Liggett CM, Knight R, Gordon JI. The human microbiome project. Nature. 2007;449(7164):804-810.

8. Lozupone C, Knight R. UniFrac: a new phylogenetic method for comparing microbial communities. Appl Environ Microbiol. 2005;71(12):8228-8235.

9. Naik S, Bouladoux N, Wilhelm C, et al. Compartmentalized control of skin immunity by resident commensals. Science. 2012;337(6098):1115-1119.

10. Yoshimura M, Nakagami G, lizaka S, et al. Microclimate is an independent risk factor for the development of intraoperatively acquired pressure ulcers in the park-bench position: A prospective observational study. Wound Repair Regen. 2015;23(6):939-947.

11. Yusuf S, Okuwa M, Shigeta $Y$, et al. Microclimate and development of pressure ulcers and superficial skin changes. Int Wound J. 2015;12(1):40-46.

12. Gefen A. How do microclimate factors affect the risk for superficial pressure ulcers: a mathematical modeling study. J T Viability. 2011;20(3):81-88.

13. Grice EA, Segre JA. The skin microbiome. Nat Rev Microbiol. 2011;9(4):244-253.

14. Junkin J, Selekof JL. Prevalence of incontinence and associated skin injury in the acute care inpatient. J Wound Ostomy Continence Nurs. 2007;34(3):260-269.

15. Allman RM. Pressure ulcers among the elderly. N Engl J Med. 1989;320(13):850-853.

16. Brandeis $G H$, Ooi WL, Hossain M, Morris JN, Lipsitz LA. A longitudinal study of risk factors associated with the formation of pressure ulcers in nursing homes. J Am Geriatr Soc. 1994;42(4):388-393.

17. Allman RM, Goode PS, Patrick MM, Burst N, Bartolucci AA. Pressure ulcer risk factors among hospitalized patients with activity limitation. JAMA. 1995;273(11):865-870.

18. Reed RL, Hepburn K, Adelson R, Center B, McKnight P. Low serum albumin levels, confusion, and fecal incontinence: are these risk factors for pressure ulcers in mobility-impaired hospitalized adults? Gerontology. 2003;49(4):255-259.

19. Keshavarz Valian $H$, Khoshabe Abdollah Kenedy L, Nateghi Rostami M, Miramin Mohammadi A, Khamesipour A. Role of Mycobacterium vaccae in the protection induced by first generation Leishmania vaccine against murine model of leishmaniasis. Parasitol Res. 2008;103(1):21-28.

20. Redel H, Gao Z, Li H, et al. Quantitation and composition of cutaneous microbiota in diabetic and nondiabetic men. J Infect Dis. 2013;207(7):1105-1114.

21. Zhang $M$, Jiang $Z$, Li D, et al. Oral antibiotic treatment induces skin microbiota dysbiosis and influences wound healing. Microb Ecol. 2015;69(2):415-421.

22. Fierer $\mathrm{N}$, Hamady $\mathrm{M}$, Lauber $\mathrm{CL}$, Knight R. The influence of sex, handedness, and washing on the diversity of hand surface bacteria. Proc Natl Acad Sci US A. 2008;105(46):17994-17999.

23. Ying S, Zeng DN, Chi L, et al. The Influence of Age and Gender on Skin-Associated Microbial Communities in Urban and Rural Human Populations. PloS One. 2015;10(10):e0141842.

24. Sardo PM, Simoes CS, Alvarelhao JJ, et al. Analyses of pressure ulcer incidence in inpatient setting in a Portuguese hospital. J Tissue Viability. 2016;25(4):209-215.

25. Schoonhoven L, Bousema MT, Buskens E, pre P-sg. The prevalence and incidence of pressure ulcers in hospitalised patients in the Netherlands: a prospective inception cohort study. Int J Nurs Stud. 2007;44(6):927-935. 
26. Syed SA, Loesche WJ. Survival of human dental plaque flora in various transport media. Appl Microbiol. 1972;24(4):638-644.

27. Caporaso JG, Lauber CL, Walters WA, et al. Ultra-high-throughput microbial community analysis on the Illumina HiSeq and MiSeq platforms. Isme J. 2012;6(8):1621-1624.

28. Hildebrand F, Tadeo R, Voigt AY, Bork P, Raes J. LotuS: an efficient and user-friendly OTU processing pipeline. Microbiome. 2014;2(30).

29. Edgar RC. UPARSE: highly accurate OTU sequences from microbial amplicon reads. Nat Methods. 2013;10(10):996-998.

30. Wang Q, Garrity GM, Tiedje JM, Cole JR. Naive Bayesian classifier for rapid assignment of rRNA sequences into the new bacterial taxonomy. Appl Environ Microbiol. 2007;73(16):5261-5267.

31. Caporaso JG, Kuczynski J, Stombaugh J, et al. QIIME allows analysis of high-throughput community sequencing data. Nat Methods. 2010;7(5):335-336.

32. Love MI, Huber W, Anders S. Moderated estimation of fold change and dispersion for RNA-seq data with DESeq2. Genome Biol. 2014;15(12):550. 


\section{Supplementary material}
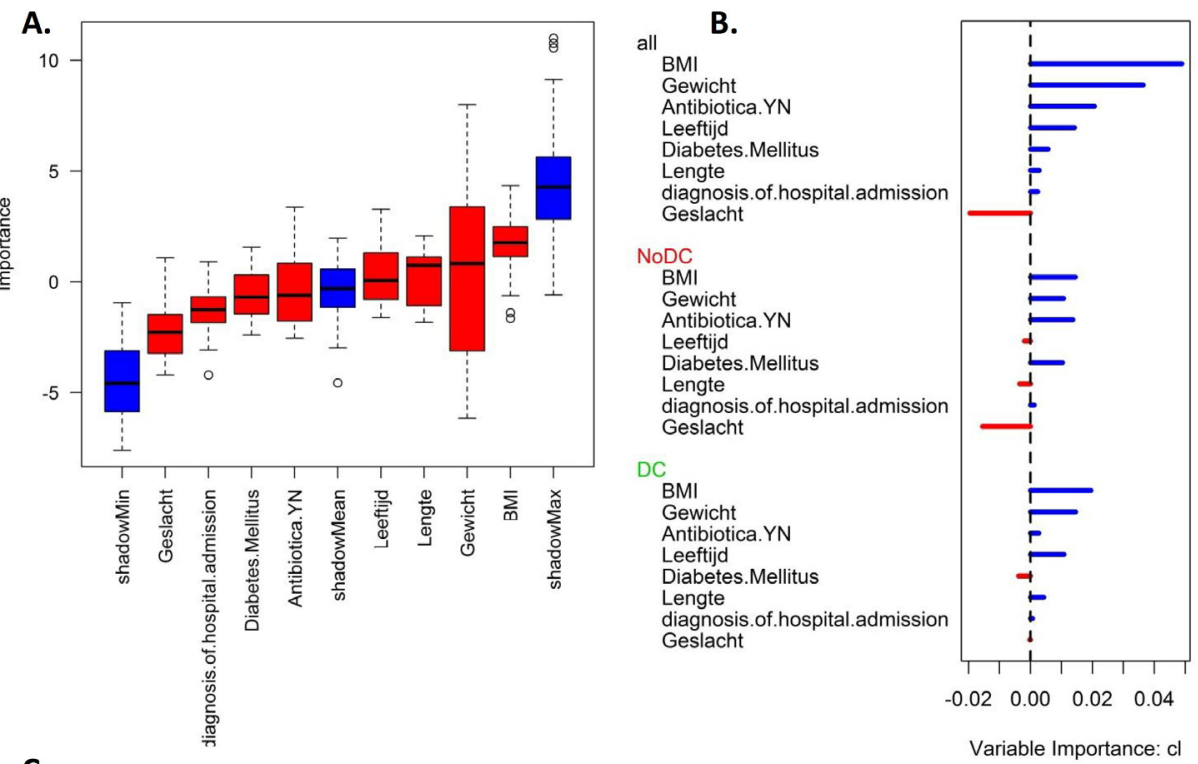

C. predicted

observed NoDC DC class.error

$$
\begin{array}{lrrr}
\text { NoDC } & 6 & 6 & 0.5000 \\
\text { DC } & 5 & 10 & 0.3333 \\
& \\
\text { Overall error rate: } 40.74 \text { 음 }
\end{array}
$$

Figure S2.1 Random forests with Boruta feature selection using clinical data. A. The importance of each feature across 1000 repeats is shown compared with the worst, best and mean randomly generated features. B. Variable importance in the random forest. C. Confusion matrix. 


\section{Chapter 3}

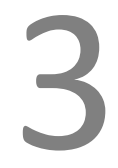

\section{A new method to evaluate the effects of shear on \\ the skin}

L.A. de Wert, D.L. Bader, C.W.J. Oomens, L. Schoonhoven, M. Poeze, N.D. Bouvy Wound repair and regeneration, 2015;23(6):885-90 


\section{Abstract}

Currently, pressure ulcer preventive strategies focus mainly on pressure redistribution. Little attention is paid to reduce the harmful effects of shear-force, because little is known about pathophysiological aspects of shear-force. Even today, no method to measure the effects of shear-force on the skin is available. Therefore, the aim of this study was to investigate the response to shear-forces in terms of analysing a non-invasive biomarker and reactive hyperaemic parameter measured at the skin of healthy participants. A physical model was developed to produce a combination of pressure and shear or pressure alone on the skin. Ten healthy male participants were included and pressure $(3.9 \mathrm{kPa})$ and a combined loading of pressure and shear $(2.4 \mathrm{kPa}+14.5 \mathrm{~N})$ was applied at the volar aspect of the forearms for 15 and 30 minutes. A Sebutape sample was used to collect IL-1 $\alpha$ and total protein non-invasively. The reactive hyperaemic parameter was derived from a Laser Doppler flowmeter. The increase in IL-1 $\alpha /$ TP ratio after a combined loading of pressure and shear for 30 minutes of $6.2 \pm 2.5$ was significantly higher compared to all other test conditions $(p<0.05)$. The increase in cutaneous blood cell flux was already significantly higher when a combined loading of pressure and shear was applied for 15 minutes compared to pressure alone. These results shows that the IL-1 $\alpha /$ TPratio and cutaneous blood cell flux can be used as robust measures of the effect of shear-force on skin in humans. Therefore, this model can be used to evaluate materials aimed at the reduction of shear. 


\section{Introduction}

The condition of pressure ulcers or decubitus represents a localized injury to skin and/ or underlying tissue, usually over a bony prominence, as a result of prolonged mechanical loading in the form of pressure, or pressure in combination with shear (EPUAP/NPUAP Guidelines 2014). By definition the pressure represents the normal force per unit area acting on the skin. Shear-forces are forces acting parallel to the skin at the interface with a support surface. Shear-forces can develop when a patient adopts a semi-reclining position ${ }^{1}$ and can slide down the bed or is transferred between support surfaces, but even occur when a patient is lying in a supine position. Prolonged mechanical loading will lead to capillary occlusion, resulting in tissue ischemia with associated localized hypoxia. This mechanism will impair the delivery of vital nutrients, such as oxygen, to the local environment of the cells. In addition, associated lymphatic occlusion will lead to accumulation of toxic metabolites. The resulting cell death will impede any remodeling process and will result in the local breakdown of soft tissues. When the mechanical load and deformation are high a more rapid process can occur, a mechanism termed direct deformation damage ${ }^{2,3}$. The presence of shear forces in association with pressure can lead to an increasing rate of cell death caused by enhanced tissue distortion and enhanced occlusion of blood vessels ${ }^{4-8}$. Indeed in a seminal study involving a swine model, Dinsdale demonstrated that the tolerable level of pressure to cause ulcers is significantly reduced in the presence of shear-forces ${ }^{9}$.

Current pressure ulcer prevention focusses on pressure relieving strategies, typically periodic repositioning and pressure redistributions ${ }^{10}$. By contrast, little attention is paid to the reduction of the harmful effects of shear-forces due both to the lack of robust measurement techniques and partly the lack of information with regards to the skin status when exposed to shear forces ${ }^{11,12}$. Consequently, the development and subsequent evaluation of materials which reduce shear forces remains a challenge for health care companies.

Ideally, a non-invasive biomarker could be utilised to measure the effect of shearforces on human skin. Potential biomarkers include cytokines that are synthesized and released after mechanical injury of the skin cells. As an example IL- $1 \alpha$, a proinflammatory cytokine that is released after injury to the keratinocytes ${ }^{13}$, has been shown to be significantly increased after pressure loading of the skin $^{14,15}$, and more importantly, increased over category I pressure ulcers ${ }^{16}$. A simple method has been developed to collect IL-1 $\alpha$ non-invasively in humans with the use of Sebutape ${ }^{17}$.

Reactive hyperaemia also represents an important physiological mechanism in response to the release of mechanical loading of the $\mathrm{skin}^{18}$. Clinically, this manifests as blanchable erythema of previously loaded skin and its presence is regularly monitored in bedridden patients ${ }^{19}$. This reactive hyperaemia is a direct result of increased cutaneous blood flow and can be measured non-invasively by a Laser Doppler 
Flowmeter ${ }^{20,21}$ or Colorimeter, the latter of which reflects the secondary effects of increased erythema ${ }^{22,23}$.

The aim of the present study was to investigate the response to shear-forces in terms of analysing a non-invasive biomarker and a selected parameter of reactive hyperaemia measured at the skin of healthy participants. This was achieved by developing a physical model incorporating the application of pressure in the absence or presence of shear-forces and measuring the IL-1 $\alpha /$ total protein-ratio and cutaneous blood cell flux after mechanical loading.

\section{Methods}

\section{Ethical considerations}

The research protocol was registered in the clinicaltrials.gov database (Number NCT02020655) and approved by the medical ethics committee of the Maastricht University Medical Centre. The study was conducted in compliance with the ethical rules for human experimentation that are stated in the Declaration of Helsinki. Written informed consent was obtained from all participants.

\section{Shear-force model}

The physical model, as illustrated in Figure 3.1, was designed to apply a combination of pressure and shear on the skin surface. This model included an anti-slip-mat (Digibuddy, Onni-tec GmbH, Germany) of $50 \times 50 \mathrm{~mm}$, which was loaded with a $1 \mathrm{~kg}$ lead weight (equivalent to a pressure of $3.9 \mathrm{kPa} / 30 \mathrm{mmHg}$ ) and a pulley that was adjustable in height and a weight of 1.7 kilogram (representing a shear-force of 19.6 $\mathrm{N})$. To produce shear-force on the skin, the $1.7 \mathrm{~kg}$ mass was connected with a line from the lead weight around the pulley. Due to the friction in the pulley, the actual force at the line was $15 \mathrm{~N}$ (which was measured by a spring balance). The angle of the line with the lead was set at $15^{\circ}$ with the skin in all participants to ensure stability of the anti-slip-mat and lead on the skin surface. Therefore, the horizontal force is equal to $15.0 \times \cos \left(15^{\circ}\right)$ which is $14.5 \mathrm{~N} / 5.8 \mathrm{kPa}$. The vertical force in this case is equal to $15.0 \times \sin \left(15^{\circ}\right)$ which is $3.9 \mathrm{~N}$, which is equivalent to a pressure, in the presence of shear of $2.4 \mathrm{kPa}$. 


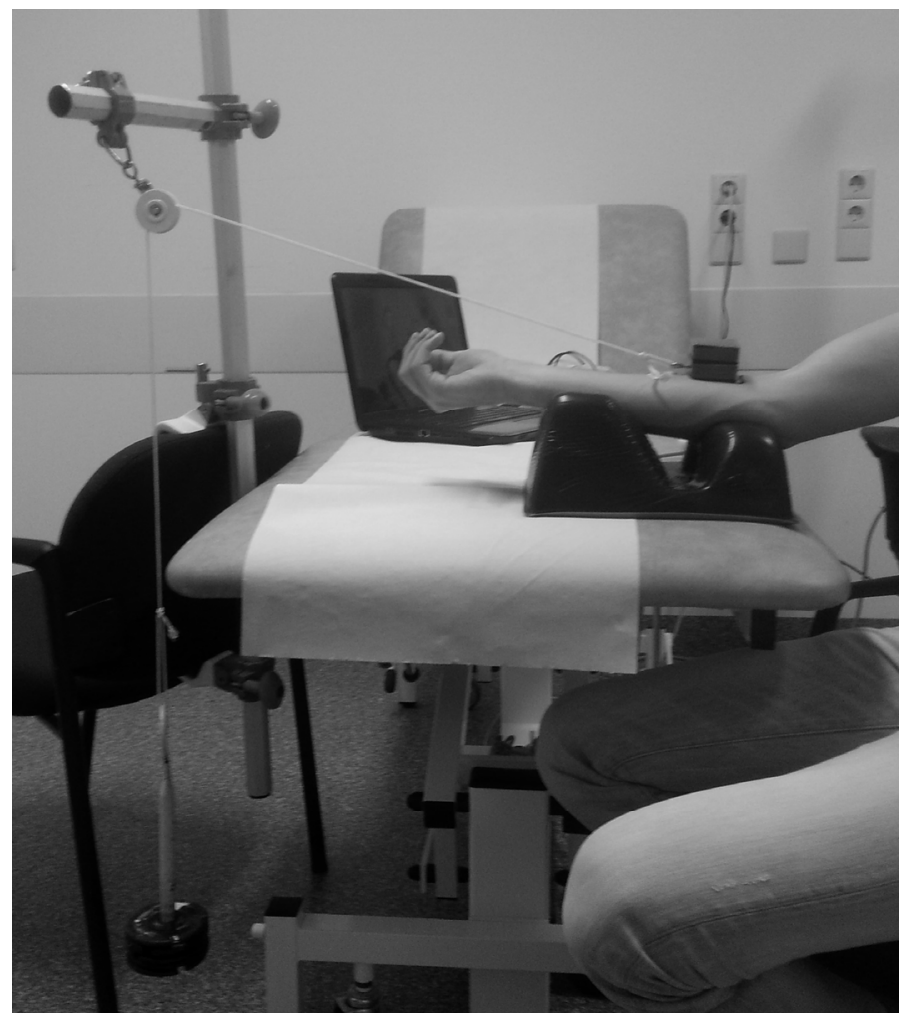

Figure 3.1 Shear-force model. An anti-slip-mat $(5 \mathrm{~cm} \times 5 \mathrm{~cm})$ was placed at the volar aspect of the forearm and exposed to a 1 kilogram lead weight. The line is set at a 15 degree angle with the skin of the participant to ensure stability of the mat and the lead weight. The weight at the end of the line is 1.7 kilogram which applies shear force.

\section{Participants and procedure}

Ten healthy Caucasian male volunteers between 18-30 years old, with a Body Mass Index (BMI) between $20-30 \mathrm{~kg} / \mathrm{m}^{2}$ and a Braden score $>20^{24}$ were eligible to participate in this study. Exclusion criteria included any history of skin diseases, trauma at the volar aspect of the forearms, muscular dystrophy, malignancy and diabetes mellitus type 1 or type 2 . After written informed consent, an appointment was made the day before the actual experiment. During this appointment, the palmar aspects of both forearms were inspected, and the researcher decided which area, $2 \mathrm{~cm}$ distal of the elbow crease, of the volar aspect of the forearms were suitable for the placement of the shear-pad. Any visible hairs in this area were removed with a razor blade to ensure optimal contact of the Sebutape (CuDerm, Dallas, USA) with the skin on the test day. A Sebutape sample, which represents a small adhesive patch, 
originally designed to collect sebum without the use of any solvents or oil, was used to absorb proteins from the skin in a non-invasive manner ${ }^{17}$.

On the test day, the participants washed their forearms with lukewarm water at the beginning of the experiment and were required to acclimatise in a relaxed state for a period of 20 minutes at room temperature $\left(21^{\circ} \mathrm{C}\right)$. Demographic data including: weight, height, blood pressure, age, medical history, medication use, smoking behaviour were all recorded.

Each participant was then required to position their non-dominant arm on a support cushion. The borders of a $50 \mathrm{~mm} \times 50 \mathrm{~mm}$ area were identified with a permanent marker on the volar aspect of both fore-arms and the adhesive side of one Sebutape was located within these areas for a two minutes period. Subsequently, a measurement of cutaneous blood cell flux was performed within the borders of the marked areas using a Laser Doppler Imaging system (LDI2-Burn Imager, Moor Instruments Ltd, Axminster, United Kingdom). The single point laser beam scanned in a raster formation to create a cutaneous blood cell flux map. The protocol was repeated on the dominant forearm. These measures represent the unloaded basal values for each parameter of both arms.

The loading phase commenced with the application of pressure (3.9 $\mathrm{kPa})$ alone over the marked area of the non-dominant forearm for a 30 minute period. At the end of this period, a Sebutape sample was attached for two minutes, followed by measurements of cutaneous blood cell flux within the borders of the marked area.

This was followed by positioning the dominant arm on the support cushion and following the 30 minute test protocol, but, in this phase, loading involved the combination of pressure $(2.4 \mathrm{kPa})$ with a shear-force of $14.5 \mathrm{~N}$. After this loading period a Sebutape sample was attached for two minutes and the cutaneous blood cell flux measurements were repeated.

On a separate day, the test protocols were repeated for a shorter period of 15 minutes. The complete test protocol is summarised in Figure 3.2.

\section{Biochemical analysis}

Two millilitre $(\mathrm{ml})$ of Phosphate Buffered Saline was added to each Sebutape sample and then sonicated for ten minutes and vortexed for a further two minutes. The extraction of proteins from the Sebutape was based on a study by Perkins et al. ${ }^{17}$ The IL-1 $\alpha$ concentration in the samples was measured by a commercially available human IL-1 $\alpha$ / IL1F1 enzyme-linked immunosorbent assay (ELISA)kit (DuoSet R\&D system) with a detection range of $3.9-250 \mathrm{pg} / \mathrm{ml}$. To correct for differences in sebum uptake during each collection period, the amount of total protein (TP) was measured using the Thermo Scientific ${ }^{\mathrm{TM}}$ Micro BCA Protein Assay Kit (Pierce Biotechnology) with a detection range of $0.5-20 \mu \mathrm{g} / \mathrm{ml}$. IL- $1 \alpha(\mathrm{pg} / \mathrm{ml})$ were divided by the TP concentrations $(\mu \mathrm{g} / \mathrm{ml})$ to calculate the IL-1 $\alpha /$ TP-ratio. 
Pressure

15 or 30 minutes
Pressure and shear

15 or 30 minutes

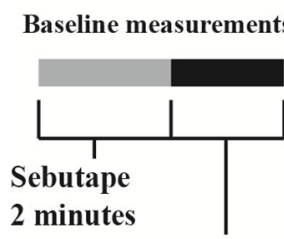

Laser doppler 30 scconds
Post- load measurements

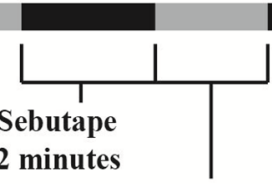

Laser doppler 30 seconds
Post- load measurements

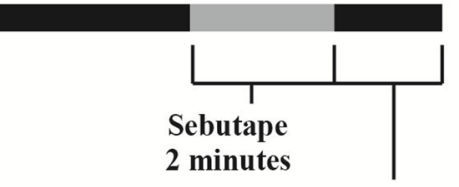

Laser doppler 30 seconds

Figure 3.2 Timeline of the experimental setup. The experiment consisted of two research days: At the first research day, the pressure and pressure combined with shear-force loading on the skin lasted 30 minutes, the other day the loading on the skin was only for 15 minutes.

\section{Biophysical analysis}

A region of interest (ROI) was selected in the cutaneous blood cell flux and customised software (Moor version 5.3) was used to calculate the mean cutaneous blood cell flux over the ROI.

\section{Statistics}

Statistical analysis was performed using GraphPad Prism 5 for Windows (GraphPad Software Inc. San Diego, CA). Increases in the two parameters were calculated by subtracting the baseline measurements from the post loaded measurements. Normality was tested by the Kolmogorov-Smirnov test. Results are expressed as mean \pm standard deviation (SD) when distributed normally or as median + range for a nonnormal distribution. In the former case a paired t-test was used to determine significance between the pairwise comparisons. A statistical significance level of $5 \%$ was prescribed $(p<0.05)$.

\section{Results}

\section{Participants}

Ten healthy Caucasian male participants were included in the study. Median age was 23 (range: 22-27) years. The mean height was $1.87 \pm 9.2$ metres; mean weight $78.0 \pm 10.9$ kilograms with a mean BMI of $22.2 \pm 1.9 \mathrm{~kg} / \mathrm{m}^{2}$. Four participants had a history of smoking with a median number of pack years of 3 (range: 2-3). None of the participants used any non-steroidal anti-inflammatory drugs during the seven days 
prior to the initial day of testing. Baseline measurements of blood pressure and each of the three objective parameters for each phase of the test protocol are summarised in Table 3.1. No statistically significant differences in the baseline values between the different phases of the test protocol were found.

Table 3.1 Baseline measurements of the participants $(n=10)$

\begin{tabular}{lcccc}
\hline & 15 minutes $\mathrm{P}$ & 15 minutes PS & 30 minutes $\mathrm{P}$ & 30 minutes PS \\
\hline Systolic blood pressure $(\mathrm{mmHg})$ & $129.4 \pm 15.9$ & $129.4 \pm 15.9$ & $125.2 \pm 19.3$ & $125.2 \pm 19.3$ \\
Diastolic blood pressure $(\mathrm{mmHg})$ & $75.4 \pm 17.4$ & $75.4 \pm 17.4$ & $75.4 \pm 7.2$ & $75.4 \pm 7.2$ \\
IL-1 $\alpha /$ TP-ratio $\left(\times 10^{-6}\right.$ ) & $1.7 \pm 1.5$ & $1.8 \pm 2.2$ & $1.2 \pm 1.4$ & $0.5 \pm 1.0$ \\
Cutaneous blood cell flux (flux per image) & $117.6 \pm 30.7$ & $114.4 \pm 33.6$ & $133.9 \pm 34.3$ & $137.2 \pm 31.4$ \\
\hline
\end{tabular}

Baseline measurements were performed before 15 minutes and 30 minutes of pressure loading $(P)$ and before 15 minutes and 30 minutes of pressure + shear-force loading (PS). Data are shown as mean \pm SD. No statistically significant differences between baseline values were found.

\section{The increase in IL-1 $\alpha$ / TP-ratio is higher after a combined loading period for 30 minutes}

After 15 minutes of applied pressure, the mean \pm SD increase from baseline value in IL-1 $\alpha$ / TP-ratio was $2.7 \pm 3.5 \times 10^{-6}$ (Figure 3.3). When the time was extended to 30 minutes, there was a further $33 \%$ increase in IL-1 $\alpha$ / TP-ratio. This difference was not statistically significant between the two periods of applied pressure $(p>0.05$. Figure 3.3).

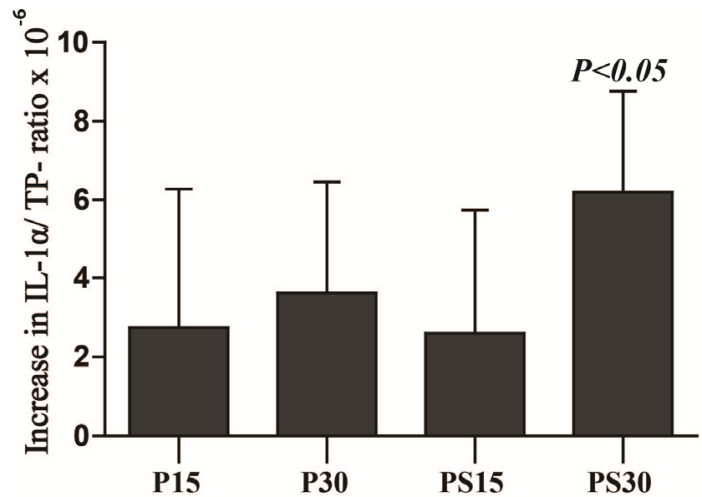

Figure 3.3 The increase in IL-1 $\alpha$ /TP- ratio after loading of the skin. P15= After pressure loading for 15 minutes, $\mathrm{P} 30=$ After pressure loading for 30 minutes, PS15= After pressure and shear- force loading for 15 minutes, PS30= After pressure and shear-force loading for 30 minutes. Data are shown as ratio's mean $\pm \mathrm{SD}$. $\mathrm{P}<0.05$ indicates a significant differences compared to all other test conditions. 
When shear was combined with pressure for 15 minutes the increase in IL-1 $\alpha /$ TPratio was $2.6 \pm 3.1 \times 10^{-6}$, a value equivalent to that for applied pressure alone ( $p>0.05$, Figure 3.3). However, when the combined loading was extended to 30 minutes, an increase in IL-1 $\alpha$ / TP-ratio to $6.2 \pm 2.5 \times 10^{-6}$ was measured, which represents a statistically significant increase from all other test conditions $(p<0.05$, Figure 3.3).

\section{The increase in cutaneous blood cell flux is higher when shear-force is added}

To study the development of reactive hyperaemia, the cutaneous blood cell flux in the loaded situations was compared to the baseline values. The mean \pm SD increase in this parameter was $50.1 \pm 30.8$ and $74.7 \pm 96.6$ arbitrary units after the application of pressure alone for 15 and 30 minutes, respectively (Figure 3.4). This difference was not statistically significant $(p>0.05)$. However, when shear was combined with pressure, the increase in cutaneous blood cell flux was markedly elevated after 15 minutes compared with the corresponding value after 30 minutes of pressure loading $(251.7 \pm 133.8$ vs. $74.7 \pm 96.6, p<0.001$, Figure 3.4). When the combined loading period was extended to 30 minutes, there was a corresponding significant increase in cutaneous blood cell flux $(508.0 \pm 234.0$ vs. $251.7 \pm 133.8, p<0.01$, Figure 3.4). 
A

\section{Baseline measurement}

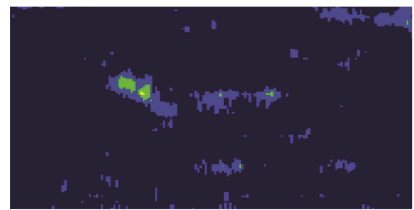

P15

PS15
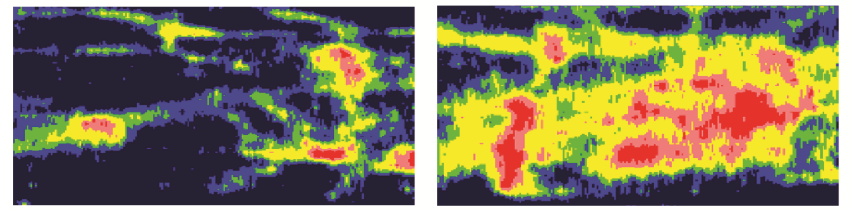

P30

PS30
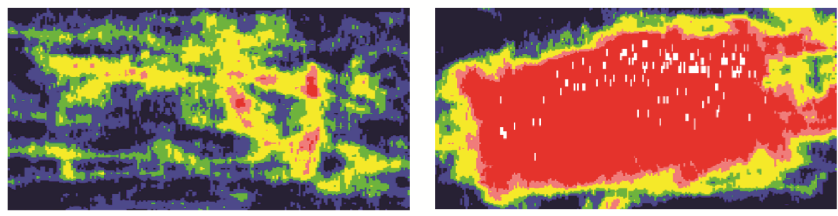

B

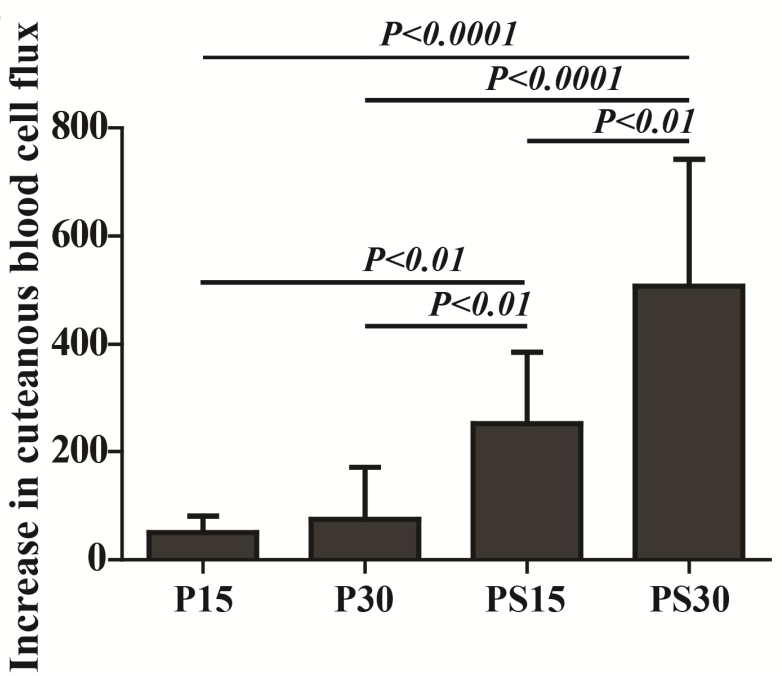

Figure 3.4 Cutaneous blood cell flux. P15= After pressure loading for 15 minutes; $\mathrm{P} 30=$ After pressure loading for 30 minutes; PS15= After pressure and shear-force loading for 15 minutes; PS30= After pressure and shear loading for 30 minutes. Cutaneous blood cell flux map created with Laser Doppler imaging. Increase in cutaneous blood cell flux compared with baseline measurements expressed in flux per image. Data are shown as mean \pm SD. 


\section{Discussion}

The aim of this study was to investigate the response of shear-forces in terms of analysing a non-invasive biomarker and a reactive hyperaemic parameter measured at the skin surface of a cohort of healthy participants. This was achieved by developing a new physical model which enabled the application of pressure in the absence or presence of shear-forces on the volar aspect of the forearm. The increase in the two objective parameters after loading of the skin namely, IL-1 $\alpha /$ TP-ratio and the cutaneous blood cell flux were assessed in ten healthy volunteers. The increase of these parameters had to be determined to assess the skin response to loading, but also to compensate for intra-individual variability.

This study is the first to demonstrate after a combined period of both pressure and shear for 30 minutes, there was a significant increase in the normalised IL-1 $\alpha$ /TP-ratio when compared to the response of applied pressure alone (Figure 3.3).

A recent study ${ }^{25}$ demonstrated that $\mathrm{IL}-1 \alpha$ is the predominant damage-associated molecular pattern in stress-damaged epithelial cells. In the skin, IL-1 $\alpha$ is produced by the healthy keratinocytes in the vital epidermis and stored in a preformed pool. If the keratinocytes are injured by mechanical or by chemical irritants ${ }^{13}$, it is rapidly released to stimulate the production of other cytokines such as IL-8 and TNF- $\alpha^{26}$. However, these down-stream cytokines are difficult to detect in healthy skin or even when the Sebutape sampling methodology was used in patients with category I pressure ulcers $^{16}$. By contrast the increase in IL-1 $\alpha /$ TP-ratio could be readily detected and could be used as a robust measure for shear. It is conceivable that tissue deformation is enhanced when shear is combined with pressure, which in turn, will lead to a higher increase in IL-1 $\alpha$ /TP-ratio, because keratinocytes are more likely to become injured when compared to that resulting from pressure alone.

Well established methods to assess the reactive hyperaemic response of skin following loading included an estimation of cutaneous blood cell flux and an index of erythema ${ }^{20-23}$. In the present study, the reactive hyperaemic response was expressed in cutaneous blood cell flux measured with Laser Doppler Flowmetry, as it provides a more robust method than an index of erythema. A significant increase in cutaneous blood cell flux following mechanical loading was measured in all test conditions. However, it was evident that the application of shear combined with pressure was shown to produce a more marked increase in cutaneous blood cell flux when compared with pressure alone. This suggests that shear-force plays a critical role in the development of reactive hyperaemia. Although reactive hyperaemia is not a direct measure of tissue damage per se, reactive hyperaemia will be influenced by the degree of tissue ischemia, the accumulation of metabolic waste products, inflammation, cell damage and eventual cell death ${ }^{18}$. It is likely that the large increase in reactive hyperaemia in the present study after a combined loading could be explained by an increase in tissue deformation and a decrease in cutaneous blood flow when shear-force is added to pressure ${ }^{4,6,7,27}$. 
At present, a number of studies investigated the effects of shear-force on the skin in humans, however, most did not quantify the magnitude of shear ${ }^{4,5,27}$. Most of these studies reported a decrease in cutaneous blood flow when shear-force is added to pressure. However, only Goossens and colleagues showed a significant decrease in cut-off pressure of the microvascular blood supply of the skin in humans ${ }^{4}$. In a computational model, with some inherent assumptions, Linder-Ganz and colleagues also predicted enhanced internal deformations in the presence of shear at the skin surface ${ }^{8}$.

Manorama et al. ${ }^{27}$ investigated the effect of shear-force on the skin in human participants and reported that shear-force decreased transcutaneous oxygen levels and blood perfusion. However, there was a large variation in the magnitude of applied shear between individuals with values ranging between 5 to $49 \mathrm{~N}$. Therefore, their methods could not be considered reproducible when determining the effects of shear on the skin in vivo.

A number of comments need to be made on the interpretation of the present findings. Although the differences in baseline levels were not statistical significant, the marked increase in IL-1 $\alpha /$ TP ratio after 30 minutes of the combined pressure and shear must be considered in the light of the corresponding low baseline values (Table 3.1). Therefore, it must be noted that the IL-1 $\alpha /$ TP ratio is dependent on a number of factors including arm-to arm variability and day-to day variability. These must be considered in the design of future studies involving this non-invasive biomarker of skin response to loading.

In the present study, the pressure of $30 \mathrm{mmHg}$ and the shear-force of $5.9 \mathrm{kPa}$ produced significant changes on the robust parameters derived from the measurement techniques. Accordingly, it provides a model system to evaluate the performance of therapeutic interventions aimed at reducing pressure and shearforces and its subsequent reactive hyperaemic, and inflammatory responses. It is accepted that the equivalent pressure and shear force imposed on the skin in our study are at lower magnitudes ( $20 \%$ and $40 \%$ respectively) of the maximum values reported by Ohura and colleagues ${ }^{12}$. However, these values are not common in on a well-designed support mattress in a clinical setting. Normally, the mean physiological pressure ranges between $30-60 \mathrm{mmHg}^{28,29}$, while the mean shear ranges between 5-20 $\mathrm{N}^{12,30}$. In addition, there were practical considerations in employing the magnitudes of pressure and shear in our model namely, the requirement for the weights employed to remain stable in the experimental setup.

This study also included young healthy volunteers, which might limit its generalizability for patients at risk for pressure ulcers which occurs mostly in the elderly, disabled and the critically $\mathrm{ill}^{31}$. For example, an impaired reactive hyperaemia might be predicted in humans with a decreased microvascular function which occurs, particularly, in patients with Diabetes Mellitus or those prescribed non-steroidal antiinflammatory drugs ${ }^{22,32,33}$. 
In conclusion, this study represent the first describing a robust method to evaluate the effects of shear-force on IL-1 $\alpha$ and reactive hyperaemic response in skin of humans. The model provides the opportunity to test materials for their shear-force reducing capacities in humans with the measurements performed non-invasively. This study also shows that a combined loading of pressure and shear-force leads to a higher increase in IL-1 $\alpha$ /TP-ratio and reactive hyperaemia compared to the application of pressure alone. Therefore, pressure ulcer preventive strategies should focus on the reduction of shear as well, as opposed to focussing on pressure relief alone. 


\section{References}

1. Reichel SM. Shearing force as a factor in decubitus ulcers in paraplegics. J Am Med Assoc. 1958;166(7):762-763.

2. Oomens CW, Bader DL, Loerakker S, Baaijens F. Pressure induced deep tissue injury explained. Ann Biomed Eng. 2015;43(2):297-305.

3. Gawlitta D, Li W, Oomens CW, Baaijens FP, Bader DL, Bouten CV. The relative contributions of compression and hypoxia to development of muscle tissue damage: an in vitro study. Ann Biomed Eng. 2007;35(2):273-284.

4. Goossens RH, Zegers R, Hoek van Dijke GA, Snijders CJ. Influence of shear on skin oxygen tension. Clin Physiol. 1994;14(1):111-118.

5. Manorama A, Meyer R, Wiseman R, Bush TR. Quantifying the effects of external shear loads on arterial and venous blood flow: implications for pressure ulcer development. Clin Biomech. 2013;28(5): 574-578.

6. Bennett L, Kavner D, Lee BK, Trainor FA. Shear vs pressure as causative factors in skin blood flow occlusion. Arch Phys Med Rehabil. 1979;60(7):309-314.

7. Zhang M, Roberts VC. The effect of shear forces externally applied to skin surface on underlying tissues. J Biomed Eng. 1993;15(6):451-456.

8. Linder-Ganz E, Gefen A. The effects of pressure and shear on capillary closure in the microstructure of skeletal muscles. Ann Biomed Eng. 2007;35(12):2095-2107.

9. Dinsdale SM. Decubitus ulcers: role of pressure and friction in causation. Arch Phys Med Rehabil. 1974;55(4):147-152.

10. Mclnnes E, Jammali-Blasi A, Bell-Syer SE, Dumville JC, Cullum N. Support surfaces for pressure ulcer prevention. The Cochrane database of systematic reviews. 2011(4):CD001735.

11. Mimura M, Ohura T, Takahashi M, Kajiwara R, Ohura N, Jr. Mechanism leading to the development of pressure ulcers based on shear force and pressures during a bed operation: influence of body types, body positions, and knee positions. Wound Repair Regen. 2009;17(6):789-796.

12. Ohura T, Takahashi M, Ohura N, Jr. Influence of external forces (pressure and shear force) on superficial layer and subcutis of porcine skin and effects of dressing materials: are dressing materials beneficial for reducing pressure and shear force in tissues? Wound Repair Regen. 2008;16(1):102-107.

13. Wood LC, Elias PM, Calhoun C, Tsai JC, Grunfeld C, Feingold KR. Barrier disruption stimulates interleukin-1 alpha expression and release from a pre-formed pool in murine epidermis. J Invest Dermatol. 1996;106(3):397-403.

14. Bronneberg D, Spiekstra SW, Cornelissen LH, et al. Cytokine and chemokine release upon prolonged mechanical loading of the epidermis. Exp Dermatol. 2007;16(7):567-573.

15. Cornelissen LH. The transport profile of cytokines in compromised human skin, PhD Thesis 2007, Eindhoven University of Technology.

16. Bronnenberg D. In vivo detection of grade I pressure ulcers, PhD Thesis 2008, Eindhoven University of Technology.

17. Perkins MA, Osterhues MA, Farage MA, Robinson MK. A noninvasive method to assess skin irritation and compromised skin conditions using simple tape adsorption of molecular markers of inflammation. Skin Res Technol. 2001;7(4):227-237.

18. Bliss MR. Hyperaemia. J Tissue Viability. 1998;8(4):4-13.

19. Konishi C, Sugama J, Sanada H, et al. A prospective study of blanchable erythema among university hospital patients. Int Wound J. 2008;5(3):470-475.

20. Bergstrand $S$, Kallman $U, E k A C$, et al. Pressure-induced vasodilatation and reactive hyperemia at different depths in sacral tissue under clinically relevant conditions. Microcirculation. 2014;21(8): 761-771.

21. Nixon J, Cranny G, Bond S. Pathology, diagnosis, and classification of pressure ulcers: comparing clinical and imaging techniques. Wound Repair Regen. 2005;13(4):365-372.

22. Nose T, Tsurumi K. Pharmacological studies on cutaneous inflammation induced by ultraviolet irradiation (1): Quantification of erythema by reflectance colorimetry and correlation with cutaneous blood flow. Jpn J Pharmacol. 1993;62(3):245-256. 
23. Suh KS, Roh HJ, Choi SY, et al. Long-term evaluation of erythema and pigmentation induced by ultraviolet radiations of different wavelengths. Skin Res Technol. 2007;13(2):154-161.

24. Schoonhoven L, Haalboom JR, Bousema MT, et al. Prospective cohort study of routine use of risk assessment scales for prediction of pressure ulcers. BMJ. 12 2002;325(7368):797.

25. Suwara MI, Green NJ, Borthwick LA, et al. IL-1alpha released from damaged epithelial cells is sufficient and essential to trigger inflammatory responses in human lung fibroblasts. Mucosal Immunol. 2014;7(3):684-693.

26. Cornelissen LH, Bronneberg D, Bader DL, Baaijens FP, Oomens CW. The transport profile of cytokines in epidermal equivalents subjected to mechanical loading. Ann Biomed Eng. 2009;37(5):1007-1018.

27. Manorama AA, Baek S, Vorro J, Sikorskii A, Bush TR. Blood perfusion and transcutaneous oxygen level characterizations in human skin with changes in normal and shear loads--implications for pressure ulcer formation. Clin Biomech. 2010;25(8):823-828.

28. Ros-Mar R, Martinez-Gamarra M. The effect of a double-regression hospital bed on interface pressure. Appl Nurs Res. 2011;24(4):e79-85.

29. Kallman U, Engstrom M, Bergstrand S, et al. The effects of different lying positions on interface pressure, skin temperature, and tissue blood flow in nursing home residents. Biol Res Nurs. 2015; 17(2):142-151.

30. Goossens RH, Snijders CJ, Holscher TG, Heerens WC, Holman AE. Shear stress measured on beds and wheelchairs. Scand J Rehabil Med. 1997;29(3):131-136.

31. Allman RM. Pressure ulcers among the elderly. N Engl J Med. 1989;320(13):850-853.

32. Fromy B, Abraham P, Bouvet C, Bouhanick B, Fressinaud P, Saumet JL. Early decrease of skin blood flow in response to locally applied pressure in diabetic subjects. Diabetes. 2002;51(4):1214-1217.

33. McLellan K, Petrofsky JS, Zimmerman G, et al. The influence of environmental temperature on the response of the skin to local pressure: the impact of aging and diabetes. Diabetes Technol Ther. 2009;11(12):791-798. 


\section{Chapter 4}

The effect of shear on skin viability in patients with

type 2 Diabetes

L.A. de Wert, M. Geerts, S.van der Brug, L. Adriaansen, M. Poeze, N. Schaper, N.D. Bouvy

Submitted 


\section{Abstract}

\section{Background}

Shear is a major risk factor in the development of diabetic foot ulcers, but its effect on skin in patients with type 2 diabetes Mellitus (DM) remains to be elucidated. The aim was to determine skin responses to shear in DM patients with and without diabetic polyneuropathy (DNP).

\section{Methods}

The forearm skin was loaded with $14.5 \mathrm{~N}$ shear (+2.4 kPa pressure), and with $3.5 \mathrm{kPa}$ pressure for 30 minutes in 10 type 2 DM patients without DNP, 10 type 2 DM patients with DNP, and 10 healthy participants. A Sebutape collected IL-1 $\alpha$ (measure of tissue damage). A Laser Doppler flowmeter measured cutaneous blood cell flux (CBF) as a measure of the reactive hyperaemic skin response.

\section{Findings}

Reactive hyperaemia and IL-1 $\alpha$ release was significantly increased after shear loading in all three groups, and was higher compared to the responses to pressure loading.

The reactive hyperaemic response after shear loading was impaired in patients with type $2 \mathrm{DM}$ compared to healthy participants, but did not differ between patients with and without DNP. The reactive hyperaemic response was negatively correlated with the blood glucose level but did not correlate with the DNP severity score.

\section{Interpretation}

Shear is important in the development of tissue damage, but the reparative responses to shear is impaired in patients with type 2 DM. DNP was not associated with altered skin responses, suggesting that loss of protective sensation to sense shear to skin remains a key factor in the development of diabetic foot ulcers in patients with DNP. 


\section{Introduction}

Poorly healing diabetic foot ulcers, one of the most feared complications of diabetes mellitus (DM), are usually caused by a close interplay of biomechanical, neurological, and frequently also vascular abnormalities ${ }^{1}$. In patients with diabetic neuropathy (DNP) structural and functional abnormalities in the foot, in combination with an altered gait, lead to increased plantar foot pressures and increased shear during walking. The harmful effects of elevated pressure on skin and subcutaneous tissues are relatively well defined and elevated plantar pressures predict future ulceration ${ }^{2}$. Several lines of evidence suggest that not only the biomechanical loading on skin and subcutaneous structures is increased in these patients but also that several reparative processes are also impaired in diabetes ${ }^{3}$. Once local pressure is relieved, the skin microvasculature reacts with an hyperaemic response in order to increase local blood flow, which is important for tissue repair ${ }^{4,5}$. The hyperaemic response of the fore-arm skin to pressure was in one study diminished in patients with type 2 diabetes, probably contributing to impaired tissue repair after mechanical stress ${ }^{6}$. In addition to pressure, elevated shear plays also an important role in causing tissue damage ${ }^{7-9}$, but reliable measurement of shear on e.g. the surface of the foot during walking is a difficult challenge. However, local elevations in plantar shear are associated with callus in the foot ${ }^{10}$, which in its turn is a marked risk factor for future ulceration in earlier studies ${ }^{11,12}$.

In healthy tissues, damage such as pressure and/or shear will elicit the release of 'Damage-associated molecular patterns (DAMPs)', endogenous molecules that play a pivotal role in the early steps of tissue repair ${ }^{13}$. Animal studies suggest that the release of these immunological signalling molecules after noxious stimuli is impaired in diabetes $^{3}$. Well known DAMPs in the epidermis are interleukin-1 $\alpha(\mathrm{IL}-1 \alpha)$, interleukin33 (IL-33), human mobility group box-1 (HMGB1), ATP and DNA strains ${ }^{14}$. IL-1 $\alpha$ is an early marker of mechanically induced skin damage in the skin, because it is rapidly released from the keratinocytes when they are injured ${ }^{15,16}$ and can be used as a marker to assess the loaded skin status ${ }^{17-19}$. The effect of DM and DNP on DAMP production of the human skin in humans is, as far as we know, not well studied yet. Moreover, little is known about the responses of the diabetic skin to shear and the influence of neuropathy on this response.

Obtaining more insight in the microvascular and immunological response of the diabetic skin to shear could facilitate the development of more fundamental strategies in preventing foot ulceration and stimulating (early) wound healing. As previously described ${ }^{7}$, we developed a model to apply shear in combination with pressure on the forearm in humans enabling us to study both the local reactive hyperaemic and immunological response of the skin to pressure and shear. The aim of this study was therefore to determine these responses to shear loading (combined with pressure), or pressure loading alone in healthy volunteers and patients with type 2 DM with and without DNP. 


\section{Methods}

\section{Ethical considerations}

The research protocol was approved by the medical ethics committee of the Maastricht University Medical Centre and registered in the clinicaltrials.gov database (Number NCT02348294). The study was conducted in compliance with ethical rules for human experimentation that are stated in the Declaration of Helsinki and monitored according to the principles of Good Clinical Practice.

\section{Participants}

Thirty participants with an age between 40-75 year were invited to participate in this study. Participants were divided in three groups of ten participants each: healthy volunteers (control), patients with type $2 \mathrm{DM}$ but without symmetrical sensorimotor polyneuropathy (DM2 PNP-), and patients with type 2 DM with symmetrical sensorimotor polyneuropathy (DM2 PNP+). The groups were matched for age, sex and body mass index (BMI).

Patients with DM type 2 were recruited at the outpatient diabetes clinic of the department of Internal Medicine of the Maastricht University Medical Centre. Written informed consent was obtained from all participants. Exclusion criteria included: any active skin diseases (such as: psoriasis and eczema), auto- immune diseases, liver or renal insufficiency, peripheral arterial diseases (ankle brachial index $<0.9$ ), and a poor glycaemic control in the last three months (HbA1c>11.0\%). Participants were not allowed to use any corticosteroids or a non-steroidal anti-inflammatory drug at least seven days before the experiment. In addition, participants were not allowed to smoke or drink caffeine and/or alcohol during the test day.

\section{Physical examination}

The diagnosis of DNP was based on a standardised clinical neurological examination (CNE) and its presence as well its severity were assessed using a validated scoring system $^{20}$. This clinical scoring system corresponds well with the results of neurophysiological examination and has acceptable sensitivity and specificity for the diagnosis of DNP when a cut-off point $>4$ is used ${ }^{21}$. A CNE score of $0-4$ indicated absence of DNP, 5-14 moderate DNP, and 15-33 severe DNP. The clinical examination consisted of assessment of lower extremities muscle strength (plantar flexion foot, dorsiflexion digitorum 1), Achilles tendon reflex and sensory function of the feet and legs (vibration sense, pinprick sense, light touch sense and position sense). Peripheral arterial disease was ruled out based on history (no complaints or previous vascular surgery), presence of all peripheral arterial pulses and a Doppler ankle brachial index $>0.9$. 


\section{Procedure}

Participants were tested at room temperature after an acclimatisation period of 20 minutes. The borders of the test area $(50 \mathrm{~mm} \times 50 \mathrm{~mm}$ ) were marked on the volar aspect of both forearms at two centimetres of the centre of the cubital fossa. Baseline measurements of the unloaded skin within the marked area were performed at both arms. First, a small adhesive patch of Sebutape (Cuderm Corp, Dallas, TX) was attached to the skin for two minutes to collect proteins from the skin. Sebutape, is a validated and reliable technique designed to absorb sebum and proteins from the skin surface $^{22}$.

Subsequently, the area was scanned back and forth with a single point laser beam (infrared $785 \mathrm{~nm}$ wavelength, MoorLDI2-Burn Imager, Moor Instruments Ltd, Axminster, United Kingdom) to create a cutaneous blood cell flux map. In this map, a region of interest (ROI) of $30 \times 30 \mathrm{~mm}$ in the centre marked area was selected and the mean velocity of the red blood cells was calculated with the Moor software version 5.3 expressed in the cutaneous blood cell flux (CBF) in arbitrary units (AU).

Then, the participants were requested to put both forearms on support cushions and loading was applied for 30 minutes on the designated area on both arms at the same time; one arm was loaded with $3.5 \mathrm{kPa}$ pressure (pressure alone); the other arm was loaded with $14.5 \mathrm{~N}$ shear (combined with $2.4 \mathrm{kPa}$ pressure) ${ }^{7}$. This model, which enabled us to apply pressure and shear, was described in detail elsewhere ${ }^{7}$.

Post- load measurements with the Sebutape and Laser Doppler were performed on both arms directly after loading and repeated after 5, 10, 15, 20 and 60 minutes. In each participant, the arm which received pressure and the arm which received shear (combined with pressure) was randomized.

\section{Biochemical analysis}

The extraction of proteins from the Sebutabe was based on a study by Perkins and colleagues $^{22}$. Briefly, two millilitre $(\mathrm{ml})$ of Phosphate Buffered Saline was added to each Sebutape sample, sonicated for ten minutes and vortexed for two minutes.

IL-1 $\alpha$, EGF and IL-33 concentrations in the samples were determined by commercially available enzyme-linked immunosorbent assays (ELISA) kits (DuoSet R\&D system) with a detection range of $3.9-250 \mathrm{pg} / \mathrm{ml}$ for IL-1 $\alpha$ and EGF, and a detection range of $5.9-1500 \mathrm{pg} / \mathrm{ml}$ for IL-33. To determine IL-6 concentrations in our samples, ELISA essay with a detection range of $7.8-1000 \mathrm{pg} / \mathrm{ml}$ was used.

\section{Statistics}

Statistical analysis was performed using GraphPad Prism 5 (GraphPad Software Inc. San Diego, CA) for windows 10 . Normality was tested with the D'Agostino Pearson Omnibus test. Data are expressed as mean with standard deviation (SD) when 
normally distributed or as median + interquartile range (IQR) when not normally distributed.

The parametric paired t-test or non-parametric Wilcoxon signed rank test was used to determine significance between pairwise comparisons. The parametric one-way ANOVA test followed by a Bonferroni post hoc correction or the non- parametric Kruskal-Wallis test followed by a Dunn multiple comparison test was used to determine significance between the groups. The parametric Pearson $r$ or the nonparametric Spearman rank test was used to determine the correlation between different variables. A $p$ value less than 0.05 was considered statistically significant.

\section{Results}

All experiments were performed between April 2015 until January 2016. In total, 30 participants were included and every participant finished the test day without any dropouts reported. The clinical characteristics of the participants are presented in Table 4.1. In summary, we studied elderly participants with overweight and the three groups were well matched for age, sex and BMI. Most participants in the DM PNP+ group had moderate polyneuropathy based on clinical neurological examination. Glycaemic control was suboptimal in most participants with DM with elevated Hba1c and elevated blood glucose levels in many during the experiment.

Table 4.1 Baseline characteristics of the participants $(n=30)$

\begin{tabular}{lcccc}
\hline & $\begin{array}{c}\text { DM PNP- } \\
(\mathbf{n}=\mathbf{1 0})\end{array}$ & $\begin{array}{c}\text { DM PNP+ } \\
(\mathbf{n}=\mathbf{1 0})\end{array}$ & $\begin{array}{c}\text { Control } \\
(\mathbf{n}=\mathbf{1 0})\end{array}$ & P-value \\
\hline Age (years) & $61.0 \pm 6.4$ & $65.2 \pm 4.8$ & $64.5 \pm 4.1$ & NS \\
Males-no (\%) & $6(60.0)$ & $6(60.0)$ & $5(50.0)$ & NS \\
Length & $168.5 \pm 9.9$ & $172.6 \pm 11.6$ & $171.8 \pm 10.5$ & NS \\
Weight & $79.2 \pm 15.9$ & $86.1 \pm 17.6$ & $80.6 \pm 12.6$ & NS \\
BMI-kg/m & $27.9 \pm 3.7$ & $28.6 \pm 2.9$ & $27.2 \pm 2.9$ & NS \\
Blood pressure-systolic & $143.9 \pm 14.5$ & $149.2 \pm 20.0$ & $139.5 \pm 16.7$ & NS \\
diastolic & $80.0 \pm 5.7$ & $81.0 \pm 10.8$ & $81.3 \pm 11.1$ & NS \\
Valk score- median (IQR) & $1.0(0-2)$ & $12.5(6.8-21.0)$ & $0(0-0)$ & Yes* \\
Glucose (mmol/l) & $8.9 \pm 1.9$ & $10.1 \pm 4.3$ & $5.7 \pm 1.1$ & Yes** \\
HbA1c (\%) & $8.2 \pm 1.0$ & $7.6 \pm 0.9$ & N.D. & NS \\
Creatinine & $80.5 \pm 17.3$ & $83.3 \pm 14.8$ & $76.3 \pm 15.5$ & NS \\
\hline
\end{tabular}

DM PNP- indicates the diabetes mellitus without polyneuropathy group. DM PNP+ indicates the diabetes mellitus with polyneuropathy group. *DM PNP- vs. control $\mathrm{P}<0.05$ and $\mathrm{DM} P N P+$ vs. control $\mathrm{P}<0.01$. ** DM PNP- vs. DM PNP+ $\mathrm{P}<0.01$ and DM PNP- vs. control $\mathrm{P}<0.001$

\section{Cutaneous blood cell flux}

After the application of shear (combined with pressure) for 30 minutes, there was a statistically significant increase in CBF (reactive hyperaemic response) in all three 
groups (Figure 4.1A). In all three groups, the CBF (in arbitrary units $A U$ ) was significantly increased immediately after unloading (time point 0 ) compared to baseline: in the healthy group (mean 492.5 SD 155.6 vs 82.0 SD 20.8, $P<0.0001$ ), in the DM PNP- group (mean 296.2 SD 142.7 vs 91.8 SD 19.1, $\mathrm{P}<0.001$ ) and in the DM PNP+ group (mean 264.8 SD 134.2 vs. 89.5 SD 20.2, $\mathrm{P}<0.01$ ).

In addition, this reactive hyperaemic response was $66.3 \%$ higher in the control group compared to the CBF of the DM PNP-group $(\mathrm{P}<0.05)$ and $86.0 \%$ higher compared to the $C B F$ of the DM PNP+ group $(P<0.01)$ (Figure $4.1 \mathrm{~A})$. No differences were observed in the reactive hyperaemic response between the DM patients with and without DNP. After 5 minutes, the CBF of the control group remained significantly higher compared with the CBF of the DM PNP+ group $(P<0.05)$. No statistically significant differences were observed between the groups at the other time points.

After the application of pressure alone, the CBF immediately after unloading (time point 0 , in $A U$ ) was significantly higher compared to baseline in the control group (mean 109.5 SD 36.8 vs. 84.1 SD 22.7, P<0.05) and the DM PNP+ group (mean 92.8 SD 25.2 vs. 77.1 SD 16.4, $P<0.05)$. In the DM PNP-group the application of pressure alone did result in numerically higher $\mathrm{CBF}$ at time point 0 , but this was not statistically significant (mean 90.4 SD 25.1 vs. 83.5 SD 14.8, P>0.05). No statistically significant differences were observed in the post-load reactive hyperaemic response between the three groups after the application of pressure alone (Figure 4.1B).
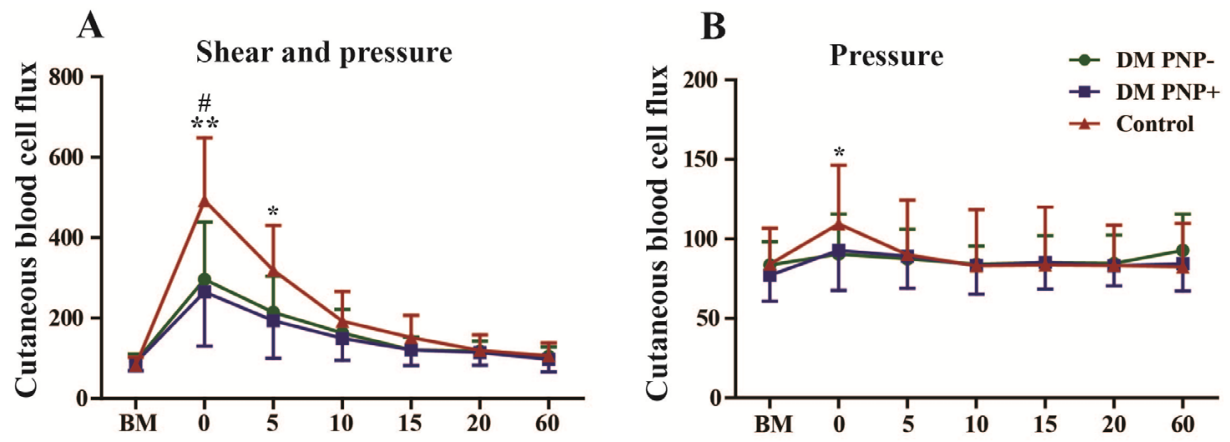

Figure 4.1 Cutaneous Blood Cell flux in arbitrary units (AU) presented as mean \pm SD. BM indicates baseline measurement before loading. $0,5,10,15,20,60$ indicates the time points after loading of the skin (in minutes). Cutaneous blood cell flux measurements after the application of pressure and shear. * Indicates DM PNP+ vs. Control $\mathrm{P}<0.05$ at this time point ** DM PNPvs. Control $\mathrm{P}<0.05$ and $\mathrm{DM} P N P+$ vs. Control $\mathrm{P}<0.01$. \# indicates a statistical significant value of $\mathrm{P}<0.0001$ in the control group, $\mathrm{P}<0.001$ in the DM PNP- group and $\mathrm{P}<0.01$ compared to their own baseline measurements. Cutaneous blood cell flux measurements after the application of pressure alone. * Indicates a statistical significant value of $\mathrm{P}<0.05$ at this time point of the DM PNP+ group or the control group compared with their own baseline measurement. No statistically significant differences were measured between the groups. 


\section{Cytokine release}

Immediately after unloading of the skin with shear (combined with pressure), the median skin IL-1 $\alpha$ concentration $(\mathrm{pg} / \mathrm{ml})$ was increased compared to baseline in all three groups, in the control group 57.0 (29.5-167.1) vs. $19.1(12.4-43.4(P<0.01)$, the DM PNP- group 50.5 (25.6-143.4) vs. 9.7 (5.8-16.4), $(\mathrm{P}<0.01)$ and the DM PNP+ group 43.5 (21.3- 61.6) vs. 15.5 (10.6-33.8) $(P<0.01)$.

The same pattern was seen after the application of pressure alone, with an increase in the median IL-1 $\alpha$ concentration in the control group 36.0 (26.8-55.4) vs. 15.4 (12.431.5) $(P<0.01)$, the DM PNP-group $32.2(11.4-76.3)$ vs $8.6(0.8-21.9)(P<0.01)$ and the DM PNP+ $37.2(19.5-76.6)$ vs. $14.5(6.0-36.1)(P<0.01)$. No statistically significant differences were observed in the post-load IL-1 $\alpha$ release between the three groups (Figure 4.2 and 4.3).

Concentration of IL-33, IL-6, EGF were not detectable, or only detectable in very low quantities in the samples, therefore they could not be used to assess the skin response to loading in this study.
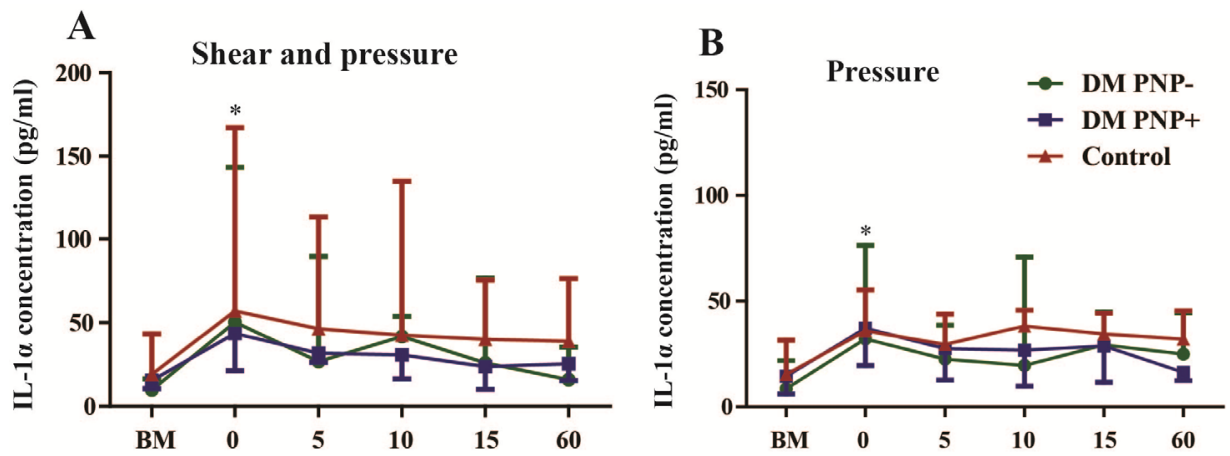

Figure 4.2 IL-1 $\alpha$ concentration $(\mathrm{pg} / \mathrm{ml})$ presented as median and IQR. BM indicates baseline measurement before loading. 0, 5, 10, 15, 60 indicates the time points after loading of the skin (in minutes). IL-1 $\alpha$ concentration measurements after the application of pressure and shear. * Indicates a statistical significant value of $\mathrm{P}<0.01$ in all three groups compared with their own baseline measurement. No statistically significant differences were measured between the groups. IL-1 $\alpha$ concentration measurements after the application of pressure and shear. ${ }^{*}$ Indicates a statistical significant of $\mathrm{P}<0.01$ in all three groups compared with their own baseline measurement. 
A

Shear and pressure

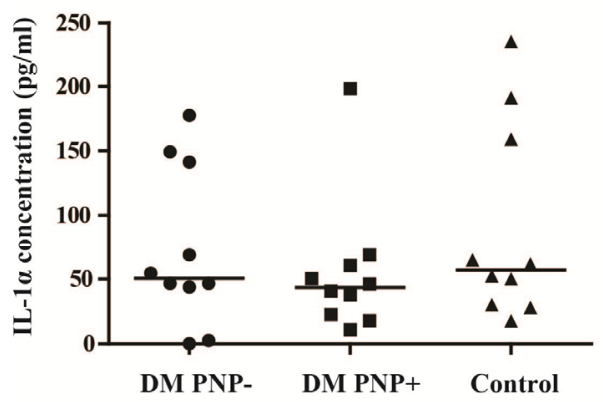

B

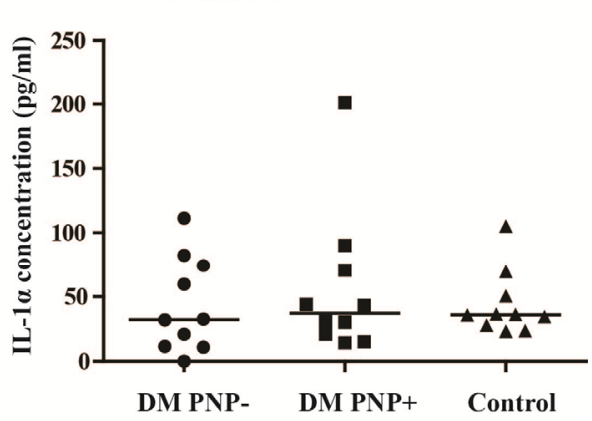

Figure 4.3 A. IL-1 $\alpha$ concentration directly after pressure and shear loading (time point 0 ). The bar indicates the median, while a dot represents the IL-1 $\alpha$ concentration in one participant. No statistically significant differences were measured between the groups. B. IL-1 $\alpha$ concentration directly after pressure alone (time point 0 ). The bar indicates the median, while a dot represents the IL-1 $\alpha$ concentration in one participant. No statistically significant differences were measured between the groups.

\section{Correlation IL-1 $\alpha$, reactive hyperaemia and glucose levels}

There was a negative correlation $(r=-0.5,95 \% \mathrm{Cl}-0.8$ to $-0.01, \mathrm{P}=0.04)$ between baseline glucose levels and CBF directly after pressure and shear loading in patients with type 2 DM with and without DNP (Figure 4.4A).

Not statistically significant correlations were found between the clinical neuropathy (CNE) score and CBF responses; IL-1 $\alpha$ concentration and CBF; baseline glucose levels and IL-1 $\alpha$ concentration, the CNE score and IL-1 $\alpha$ concentration immediately after shear combined with pressure loading (time point 0 ) (Figure 4.4B,C,D,E). 
A

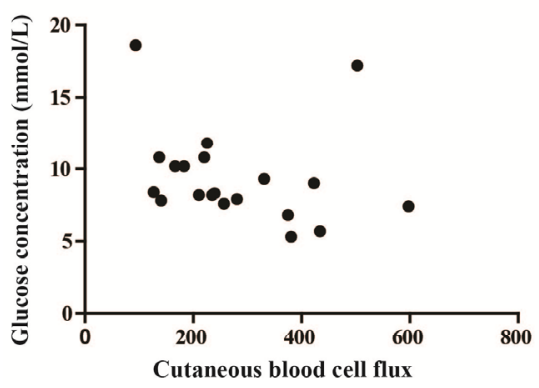

B

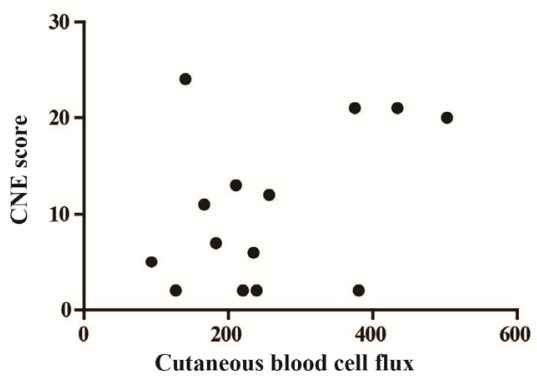

C

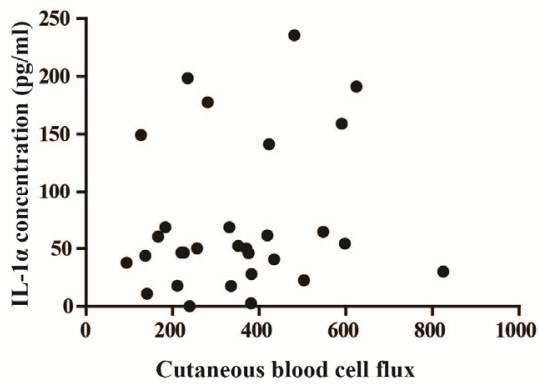

D

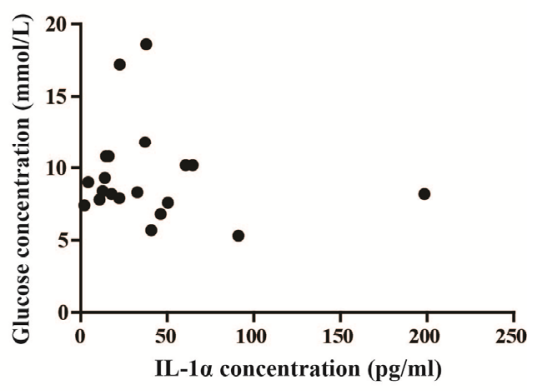

$\mathbf{E}$

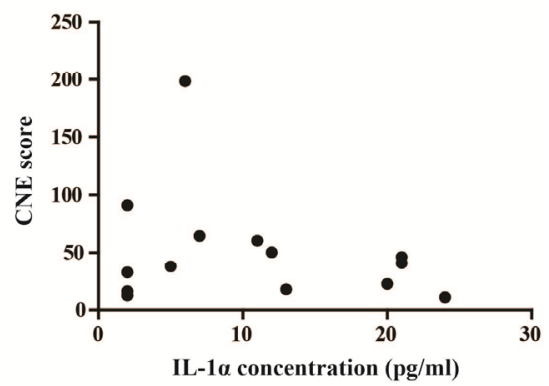

Figure 4.4 A. Correlation between baseline glucose concentration in blood $(\mathrm{mmol} / \mathrm{L})$ and cutaneous blood cell flux directly after shear combined with pressure loading (time point 0 ) in patients with type $2 \mathrm{DM}(-0.5(95 \% \mathrm{Cl}-0.8$ to $-0.01, \mathrm{P}=0.04)$. Correlation was tested with the Spearman rank test. B. Correlation between CNE score and cutaneous blood cell flux directly after shear combined with pressure loading (time point 0 ) in patients with a CNE score of $\geq 1$ were $(0.2$, $95 \% \mathrm{Cl}-0.4$ to $0.7, \mathrm{P}=0.4$ ). Correlation was tested with the Spearman rank test. C. Correlation between IL-1 $\alpha$ concentration $(\mathrm{pg} / \mathrm{ml}$ ) and cutaneous blood cell flux (AU) directly after shear combined with pressure loading (time point 0$)(0.2,95 \% \mathrm{Cl}-0.2$ to $0.5, \mathrm{P}=0.4)$. Correlation was tested with Spearman rank test $(P>0.05)$. D. Correlation between baseline glucose concentration in blood $(\mathrm{mmol} / \mathrm{L})$ and $\mathrm{IL}-1 \alpha$ concentration $(\mathrm{pg} / \mathrm{ml})$ directly after shear combined with pressure loading (time point 0 ) in patients with type $2 \mathrm{DM}(-0.1,95 \% \mathrm{Cl}-0.5$ to $0.4, P=0.6)$. Correlation was tested with the Spearman rank test $(P>0.05)$. E. Correlation between VALK score and $\mathrm{IL}-1 \alpha$ concentration $(\mathrm{pg} / \mathrm{ml})$ directly after shear combined with pressure loading (time point 0$)(95 \% \mathrm{Cl}-0.6$ to $0.5, \mathrm{P}=0.7)$. Correlation was tested with the Spearman rank test $(P>0.05)$. Only participants with a VALK score of $\geq 1$ were included in the analysis. 


\section{Discussion}

Increased biomechanical loading plays a pivotal role in foot ulceration in patients with type 2 DM and although the response to various stimuli has been investigated in multiple studies, the effect of increased biomechanical loading- and in particular shear- are less well studied in DM patients. To the best of our knowledge, this study was the first to investigate the immunological and microcirculatory responses of the skin to shear (combined with pressure) loading in patients with type 2 DM with and without DNP.

In line with our previous study in healthy participants ${ }^{7}$, the hyperaemic and immunological response of the skin to the application of shear (combined with pressure) was higher compared to the responses to pressure without shear in the three groups studied.

The reactive hyperaemic response to shear (combined with pressure) was impaired in patients with type $2 \mathrm{DM}$ in comparison to healthy volunteers, but did not differ between the patients with type 2 DM with or without DNP. In contrast, no differences in the post-load IL-1 $\alpha$ release (a measure of tissue damage) was observed between the three groups.

Reactive hyperaemia is a physiological response that occurs as a reaction to tissue ischemia and damage in order to increase or restore oxygen and metabolic delivery, and is considered to be an important response to protect the skin against ischemic skin damage ${ }^{4}$. The impaired microvascular response to shear (combined with pressure) in our patients with type $2 \mathrm{DM}$ is in line with the abnormal vasodilator responses of the microvasculature to various noxious stimuli such as pressure loading, trauma and heat ${ }^{23,24}$. This abnormal responsiveness of the type 2 diabetic microcirculation is probably related to both impaired endothelial dependent and endothelial independent vasodilation ${ }^{25,26}$. The effect of neuropathy is less clear and may depend on the stimuli applied. The hyperaemic response to heat is already disturbed very early in the course of the development of type $2 \mathrm{DM}^{24,27}$ and is probably, at least part, related to small fibre neuropathy ${ }^{28}$. The effect of large fibre neuropathy on vasodilator responses to local pressure is less well defined, but the reactive hyperaemic response to pressure was reduced in two studies in patients with $D N P^{6,29}$. It is interesting to note that we did not observe differences after the application of pressure alone between the patients with type $2 \mathrm{DM}$ and the healthy controls, nor within the two groups of type 2 DM patients with and without DNP in our study. However, only one and relatively mild pressure stimulus was applied and we cannot exclude that with higher pressures abnormal responses might have been observed, but the main aim of our study was to investigate response to shear (combined with pressure), and not to pressure alone. Our data underscores the importance of shear in the development of tissue damage, given the markedly increased hyperaemic response and- to somewhat lesser extent- immunological responses compared to pressure alone. Unfortunately, although increased shear 
forces are thought to play an important role in the development of foot ulcers in patients with type $2 \mathrm{DM}$, these forces are currently very difficult to measure in this context.

The post-load IL-1 $\alpha$ release was significantly increased after the application of shear (combined with pressure), and after- to a lesser extent- pressure alone in all three groups. However, in contrast to the CBF readings, no significant differences were observed in the post-load IL-1 $\alpha$ release between healthy volunteers, or patients with type 2 DM. IL-1 $\alpha$ is an early marker of mechanically induced skin damage in the skin, because it stored in the keratinocytes and will be rapidly released when they become injured ${ }^{15,16}$. In addition, IL-1 $\alpha$ is one of the most important DAMPs in the $\operatorname{skin}^{30}$ and has an essential role in maintaining a normal skin barrier. The results of our study suggest that direct skin damage caused by shear (combined with pressure) did not differ between patients with type 2 DM with and without DNP and healthy volunteers, as the post-load IL-1 $\alpha$ concentration was the same in all three groups. Our data suggest that increased susceptibility of diabetic tissues to noxious stimuli might be more related to impaired vasodilator capacity of the microcirculation than to increased vulnerability per se. Interestingly, the amount of DNP did not affect the immunological or microvascular skin response after the application of shear in our study, in contrast, diabetic neuropathy did affect vasodilator response to local pressure in previous studies ${ }^{20,31}$. We measured the skin responses to shear in the forearm and therefore we cannot exclude that different results might have been obtained when measurements were performed in the foot (which was technically not feasible). But, as recently reviewed by Fuchs and colleagues ${ }^{32}$, the microcirculation in feet and toes can fluctuate substantially, while the forearm and lower leg have a more stable microcirculation. In addition, as we only applied one specific shear force to the skin, it is also possible that with lower shear or shorter duration differences might have been observed. However, our data underscore the fact that the loss of the protective sensation with the inability to sense excessive loading to skin remains a key factor in the development of diabetic foot ulcers in patients with DNP.

In earlier follow-up studies ${ }^{33,34} \mathrm{Hba1c}$ levels were negatively associated with capsaicin (C-nociceptive-dependent) vasodilation or heat induced vasodilation, measured with laser Doppler flowmeter. In the current study, we observed a negative correlation between the blood glucose level at the start of the experiment and the reactive hyperaemic response after shear (combined with pressure) in the patients with type 2 DM. Also in the large scale epidemiological Maastricht study, both Hba1c and fasting blood glucose level were associated with impaired microvascular function in the $\operatorname{skin}^{35}$. These data suggest that not only long-term metabolic control, but also short term metabolic perturbations can negatively affect skin microcirculatory responses to external noxious stimuli.

Our study has several limitations: Most of our patients with DNP had mild to moderate DNP based on their CNE score and different results might have been observed if only patients with severe DNP had been included. In addition, we cannot 
exclude that different results could have been obtained of the measurements were performed on the plantar surface of the foot, an area that would have been more severely affected by DNP. Finally we used Sebutape as a non-invasive sampling method of skin cytokines. It is possible that with this techniques we were not able to detect other cytokines such as IL-33, EGF and IL-6, because to low concentrations were obtained. This problem could possibly be solved by taking skin biopsies, which of course has other limitations.

In conclusion, the reactive hyperaemic skin response to shear (combined with pressure) was decreased in patients with type 2 DM. Moreover, this reactive hyperaemic skin response was negatively correlated with the ambient blood glucose value. In contrast, post-load IL-1 $\alpha$ release, presumably a measure of skin damage and vulnerability, was not altered in patients with type $2 \mathrm{DM}$. These data suggest that in type $2 \mathrm{DM}$ in particular the reparative (microcirculatory) responses to noxious stimuli are impaired, resulting in impaired damage control once the skin has been abnormally loaded. DNP was not associated with altered skin responses to shear, suggesting that it is in particular the loss of sensation that puts patients with DNP at risk for developing tissue damage. 


\section{References}

1. Boulton AJ. The diabetic foot: from art to science. The 18th Camillo Golgi lecture. Diabetologia. 2004;47(8):1343-1353.

2. Schaper NC, et al. Prevention and management of foot problems in diabetes: a Summary Guidance for Daily Practice 2015, based on the IWGDF Guidance Documents. Diab Metab Res Rev. 2016;32 Suppl 1:7-15.

3. Schaper NC, Havekes B. Diabetes: impaired damage control. Diabetologia. 2012;55(1):18-20.

4. Fromy $\mathrm{B}$, et al. Asic3 is a neuronal mechanosensor for pressure-induced vasodilation that protects against pressure ulcers. Nat Med, 2012;18(8):1205-1207.

5. Danigo A, et al. Candesartan restores pressure-induced vasodilation and prevents skin pressure ulcer formation in diabetic mice. Cardiovasc Diabetol 2015;14:26.

6. Petrofsky, J.S., et al., The influence of age and diabetes on the skin blood flow response to local pressure. Med Sci Monit. 2009;15(7):CR325-CR331.

7. de Wert LA, et al. A new method to evaluate the effects of shear on the skin. Wound Repair Regen. 2015;23(6):885-890.

8. Linder-Ganz E, Gefen A. The effects of pressure and shear on capillary closure in the microstructure of skeletal muscles. Ann Biomed Eng. 2007;35(12):2095-2107.

9. Dinsdale SM. Decubitus ulcers: role of pressure and friction in causation. Arch Phys Med Rehabil. 1974;55(4):147-152.

10. Hamatani M, et al. Factors Associated With Callus in Patients with Diabetes, Focused on Plantar Shear Stress During Gait. J Diabetes Sci Technol. 2016;10(6):1353-1359.

11. Yavuz $M$, et al. Peak plantar shear and pressure and foot ulcer locations: A call to revisit ulceration pathomechanics. Diab Care. 2015;38(11):e184-185.

12. Lord M, Hosein R. A study of in-shoe plantar shear in patients with diabetic neuropathy. Clin Biomech (Bristol, Avon). 2000;15(4):278-283.

13. Kaczmarek A, Vandenabeele P, Krysko DV. Necroptosis: the release of damage-associated molecular patterns and its physiological relevance. Immunity. 2013;38(2):209-23.

14. Bertheloot D, Latz E. HMGB1, IL-1alpha, IL-33 and S100 proteins: dual-function alarmins. Cell Mol Immunol. 2017;14(1):43-64.

15. Lee RT, et al. Mechanical deformation promotes secretion of IL-1 alpha and IL-1 receptor antagonist. J Immunol. 1997;159(10):5084-5088.

16. Wood LC, et al. Barrier disruption stimulates interleukin-1 alpha expression and release from a preformed pool in murine epidermis. J Invest Dermatol. 1996;106(3):397-403.

17. Bronneberg D, et al. Cytokine and chemokine release upon prolonged mechanical loading of the epidermis. Exp Dermatol. 2007;16(7):567-573.

18. Cornelissen $\mathrm{LH}$, et al. The transport profile of cytokines in epidermal equivalents subjected to mechanical loading. Ann Biomed Eng. 2009;37(5):1007-1018.

19. Cornelissen L. The transport profile of cytokines in compromised human skin. PhD- thesis 2008, Technical University Eindhoven.

20. Nabuurs-Franssen $\mathrm{MH}$, et al. The effect of polyneuropathy on foot microcirculation in Type II diabetes. Diabetologia. 2002;45(8):1164-1171.

21. Valk GD, et al. The assessment of diabetic polyneuropathy in daily clinical practice: reproducibility and validity of Semmes Weinstein monofilaments examination and clinical neurological examination. Muscle Nerve. 1997;20(1):116-118.

22. Perkins $\mathrm{MA}$, et al. A noninvasive method to assess skin irritation and compromised skin conditions using simple tape adsorption of molecular markers of inflammation. Skin Res Technol. 2001;7(4): 227-237.

23. Walmsley D, Wales JK, Wiles PG. Reduced hyperaemia following skin trauma: evidence for an impaired microvascular response to injury in the diabetic foot. Diabetologia. 1989;32(10):736-739.

24. Jaap AJ, et al. Reduced microvascular hyperaemia in subjects at risk of developing type 2 (non-insulindependent) diabetes mellitus. Diabetologia. 1994;37(2):214-6.

25. Johnstone MT, et al. Impaired endothelium-dependent vasodilation in patients with insulindependent diabetes mellitus. Circulation. 1993;88(6):2510-6. 
26. Nguyen TT, et al. Diabetic retinopathy is related to both endothelium-dependent and -independent responses of skin microvascular flow. Diabetes Care. 2011;34(6):1389-1393.

27. Green $A Q$, et al. Altered C-fiber function as an indicator of early peripheral neuropathy in individuals with impaired glucose tolerance. Diabetes Care. 2010;33(1):174-176.

28. Kilo S, et al. Neural and endothelial control of the microcirculation in diabetic peripheral neuropathy. Neurology. 2000;54(6):1246-1252.

29. Fromy B, et al. Early decrease of skin blood flow in response to locally applied pressure in diabetic subjects. Diabetes. 2002;51(4):1214-1217.

30. Suwara MI, et al. IL-1alpha released from damaged epithelial cells is sufficient and essential to trigger inflammatory responses in human lung fibroblasts. Mucosal Immunol. 2014;7(3):684-693.

31. Hsu PC, et al. Nailfold capillary abnormalities are associated with type 2 diabetes progression and correlated with peripheral neuropathy. Medicine (Baltimore). 2016;95(52):e5714.

32. Fuchs $D$, et al. The association between diabetes and dermal microvascular dysfunction noninvasively assessed by laser Doppler with local thermal hyperemia: a systematic review with metaanalysis. Cardiovasc Diabetol. 2017;16(1):11.

33. Rathsman B, Jensen-Urstad K, Nystrom T. Intensified insulin treatment is associated with improvement in skin microcirculation and ischaemic foot ulcer in patients with type 1 diabetes mellitus: a long-term follow-up study. Diabetologia. 2014;57(8):1703-1710.

34. Jaap AJ, et al. Microvascular function in type 2 (non-insulin-dependent) diabetes: improved vasodilation after one year of good glycaemic control. Diabet Med. 1995;12(12):1086-1091.

35. Sorensen BM, et al. Prediabetes and Type 2 Diabetes Are Associated With Generalized Microvascular Dysfunction: The Maastricht Study. Circulation. 2016;134(18):1339-1352. 



\section{Chapter 5}

Cytokine IL1 $\alpha$ and lactate as markers for tissue damage in spineboard immobilisation. A prospective, randomised open-label crossover trial

B. Hemmes, L.A. de Wert, P.R.G. Brink, C.W.J. Oomens, D.L. Bader, M. Poeze Journal of the mechanical behaviour of biomedical materials, 2017;75:82-88 


\section{Abstract}

\section{Background}

Spinal immobilisation using a rigid long spineboard is a well-established procedure in trauma care. During immobilisation, the body is exposed to high tissue-interface pressures. This may lead to a localised inflammatory response of the skin, which may be used to monitor the body's response to different types of immobilisation device.

\section{Aim}

In this study we compared the standard rigid spineboard with a new soft-layered spineboard regarding tissue-interface pressures, skin redness as an indicator of reactive hyperaemia and cutaneous IL $1 \alpha$ and lactate release.

\section{Methods}

Twelve healthy male participants were asked to lie supine on both a rigid and a soft-layered spineboard, loading the sacrum for one hour, followed by one hour in unloaded position. Tissue-interface pressures on the buttocks during loading were measured continuously using a pressure mapping mat. Cutaneous IL $1 \alpha$ and lactate concentrations were assessed using Sebutapes, during 20-minute periods. After each 20-minute period, a photo of the buttocks was taken, which was later assessed for redness by two observers.

\section{Results}

Significant differences in tissue-interface pressure and reactive hyperaemia were found between the two types of spineboard. Release of IL1 $\alpha$ and lactate were found to increase with prolonged exposure to pressure, and to decrease in the unloaded prone position. A significant relationship was found between tissue-interface pressure and reactive hyperaemia, but not with IL1 $\alpha$ nor lactate release. Time course of IL1 $\alpha$ and lactate release was similar for both types of spineboard.

\section{Conclusions}

IL1 $\alpha$ and lactate both have a strong relationship with pressure exposure time, but not with pressure magnitude. Furthermore, IL $1 \alpha$ was measured even in the absence of visible redness of the skin. The study offers the potention of biomarkers, reflecting inflammation and/or tissue metabolism, for use in assessing the effects of prolonged spineboard support. 


\section{Introduction}

The use of spinal immobilisation with long spineboards and cervical collars in the extrication and transfer of trauma patients is a well-established standard precaution in both pre-hospital and in-hospital protocols ${ }^{1,2}$. These methods are considered particularly essential for unconscious trauma patients, who are unable to maintain spinal alignment by muscle tone and thus need to be protected from any subsequent spinal injury. Although there is a general consensus that the patient should be removed from the spineboard as soon as reasonably possible $e^{1,3,4}$, the average time they are reported to be supported on a spineboard has been estimated to be approximately one hour ${ }^{5-7}$ but in some cases may be markedly longer ${ }^{6,8}$. Prolonged immobilisation on the spineboard causes significant discomfort and pain ${ }^{9}$ and, on occasions, may cause pressure ulcers to develop adjacent to bony prominences ${ }^{8}$. These ulcers are painful ${ }^{10-12}$ and debilitating for the patient ${ }^{13,14}$ and take a long time to heal $^{15-17}$, resulting in prolonged hospitalisation ${ }^{18,19}$ and reduced quality of life $e^{20-23}$.

Pressure ulcers can be caused by a number of mechanisms including oxygen and/or nutrient deprivation and cell damage caused by pressure ${ }^{24-26}$. They can be initiated either superficially at the skin surface and progress downwards or in deeper soft tissue layers adjacent to bone prominences and progress up towards the the skin surface. Lying on a rigid surface compromises the ability of the soft tissues (skin, muscles, fat) to adapt to the shape of the surface, resulting in high tissue-interface pressures $^{27,28}$, which can lead to high compressive and shear strains within the tissues $^{29-31}$. Although the relation between (high) tissue-interface pressures and the onset of pressure ulcers remains debated, whether it is either the superficial or deeptissue injury ${ }^{30}$, high tissue-interface pressures may lead to closure of capillaries, thereby depriving tissues of oxygen and other vital nutrients, which will eventually lead to cell damage. Furthermore, the high strains themselves may cause cell damage directly $^{32,33}$, adding to the risk of developing pressure ulcers. This cell damage triggers an inflammatory response involving the release of cytokines, such as IL1 $\alpha$, IL1RA and IL-8, into the skin. Previous studies ${ }^{34,35}$ showed that IL1 $\alpha$ can be detected after relatively short periods of loading time ( $<2$ hours) with its release related to the magnitude of pressure, and its release is up-regulated over sacral sites at which pressure ulcers are observed ${ }^{36,37}$. In addition, high pressures and high tissue strains can disturb the balance between oxygen delivery and consumption, thereby increasing lactate production ${ }^{38}$. Cytokines and lactate can be collected non-invasively in humans with the use of Sebutapes ${ }^{35,39,40}$, self-adhesive patches specifically developed to absorb sebum, which can yield a number of proteins including cytokines. Previous research by our group showed that lying on a rigid spineboard results in high tissue-interface pressures ${ }^{27}$, increased reactive hyperaemia ${ }^{28}$ and increased tissue strains at the sacrum ${ }^{29}$. The hypothesis for the present study was that lying on a rigid spineboard would result in an elevated release of IL1 $\alpha$ and lactate as a result of the increased tissue-interface pressures when compared to lying on a soft-layered 
spineboard. In addition, we hypothesised that there would be a relationship between the pressure-induced reactive hyperaemia and the IL $1 \alpha$ and lactate concentrations.

\section{Methods}

\section{Study design}

We performed a prospective, randomised open-label crossover study on healthy participants to evaluate a number of parameters including tissue-interface pressures, redness of the skin, and IL $1 \alpha$ and lactate concentrations as measured on both a standard rigid long spineboard and a soft-layered long spineboard. The study was approved by the Medical Research Ethics Committee of the Maastricht University Medical Centre and registered as NTR4537 in the Dutch Primary Registry.

\section{Participants}

Following the rationale presented by Julious ${ }^{41}$ for pilot studies where no prior information is available, a sample size of 12 was selected. The participants were recruited through advertisements and word of mouth. Inclusion criteria were male sex, age 20-30 years, BMI $19-25 \mathrm{~kg} / \mathrm{m}^{2}$ and Caucasian ethnicity. Exclusion criteria included individuals with a history of pressure ulcers and skin conditions such as eczema, rashes or psoriasis with local expression on the buttocks/sacrum. Written informed consent was obtained from all participants before enrolment in the study.

\section{Materials and interventions}

Participants were randomly assigned to the order in which the rigid and soft-layered spineboard were to be tested. Testing took place in two separate sessions. Two different support devices designed for prehospital trauma care were used: a standard long spineboard (Ferno-Washington, Wilmington, $\mathrm{OH}$ ), and a prototype soft-layered long spineboard ${ }^{27-29}$. All tests were done in the same climate-controlled room, which was kept at a temperature of $23.2 \pm 0.4^{\circ} \mathrm{C}$. Each session started with a 15 -minute acclimatisation period, during which the buttock remained completely unloaded.

The area of interest surrounding the bony prominence of the sacrum was marked with a surgical pen. At the end of the acclimatisation period, four Sebutapes were placed on the skin in the marked-out area, defined as top left, top right, bottom left and bottom right (Figure 5.1), and fixed in place using a custom-made pressure-roller, specifically designed to minimise the pressure onto the skin while applying the Sebutpaes $^{36}$. After one minute, each Sebutape was retrieved, placed in a coded vial and stored on dry-ice. The Sebutapes were then replaced and the participant lay supine on the spineboard for another 20 minutes, after which the Sebutapes were once again replaced. This procedure was repeated three times, allowing for a total 
loaded period of 1 hour. It was then repeated three more times at 20-minute intervals while the buttocks remained unloaded, to monitor the reperfusion during which the volunteers were allowed to either stand or lie prone. At the end of the test session, the vials with Sebutapes were placed in a $-80^{\circ} \mathrm{C}$ freezer and stored until further processing. The second test session was planned a week later, and at this session the other device was tested in a manner identical to that used in the first session.

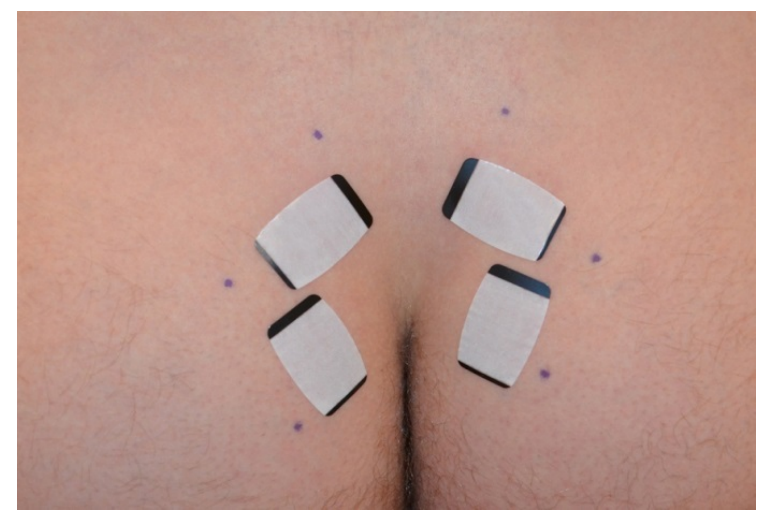

Figure 5.1 Four Sebutapes in place in the marked-out area of interest on the sacrum/buttocks for baseline measurement.

The Sebutape extraction process was based on the protocol reported by Perkins and colleagues $^{40}$. Briefly, the vials with tapes were thawed to room temperature, after which $2 \mathrm{ml}$ phosphate buffered saline was added to each vial. After 1 hour, the tapes were sonicated for $10 \mathrm{~min}$ at $20^{\circ} \mathrm{C}$, vigorously vortexed for $2 \mathrm{~min}$ and additionally mixed with a pipette tip. IL $1 \alpha$ concentration in the samples was measured using a commercially available human IL1 $\alpha$ / IL1F1 enzyme-linked immunosorbent assay (ELISA) kit (DuoSet R\&D system) with a detection range of $3.9-250 \mathrm{pg} / \mathrm{ml}$. IL1 $\alpha$ levels below the detection threshold were recorded as zero for statistical testing. The amount of total protein (TP) collected on each Sebutape was measured using a Thermo Scientific TM Micro BCA Protein Assay Kit (Pierce Biotechnology) with a detection range of $0.5-20 \mu \mathrm{g} / \mathrm{ml}$.

Tissue-interface pressures were continuously measured using a Tekscan Conformat model 5330 pressure-mapping device (Tekscan Inc, Boston, MA, USA). This system consists of a thin, easily foldable seat-sized pressure-mapping mat, equipped with 1024 capacitive sensors. This mat was placed between the participant and the spineboard at the level of the buttocks. The mat was connected to a laptop computer with special Tekscan software (version 7.20), for real-time pressure monitoring.

Lactate concentrations in the Sebutape samples were enzymatically analysed using the Cobas Fara Centrifugal Spectrophotometer (Roche Diagnostica, Basel, Switserland). 
After each 20-minute period, the back of each participant was visually inspected and the skin redness was semi-quantitatively graded. Any redness was tested for being blanchable using a clear plastic disc, so as to distinguish between blanchable and nonblanchable erythema ${ }^{36}$. Photographs of the buttocks were made to permit an independent second reviewer to quantify and qualify the presence of redness.

\section{Outcome measures}

IL1 $\alpha$ in $\mathrm{pg} / \mathrm{ml}$ and lactate in $\mathrm{mmol} / \mathrm{I}$ were measured at baseline and subsequently after every 20-minute period. Total protein (TP) was measured in $\mu \mathrm{g} / \mathrm{ml}$ at the same time points. To correct for sebum uptake by the Sebutapes, IL1 $\alpha /($ TP)- ratio was calculated as IL $1 \alpha$ concentration $(\mathrm{pg} / \mathrm{mL})$ divided by the total protein (TP) concentration $(\mu \mathrm{g} / \mathrm{ml})$. IL1 $\alpha$ /TP ratio was used as a standardised measure to compensate for inter-individual excretion differences.

Pressures in $\mathrm{mmHg}$ were recorded continuously during the loaded period for each sensor, as described in previous studies ${ }^{27,28}$. Peak pressure (in $\mathrm{mmHg}$ ) was defined as the sensor showing the highest pressure in the sacral area in the first recording of every 20-minute period. Peak Pressure Index (PPI) in $\mathrm{mmHg}$ was defined as the area of $3 \times 3$ sensors which included the sensor showing the peak pressure and which had the highest average pressure including this sensor (Figure 5.2A and 5.2B). The area was identified based on the first recording of every 20-minute period in the loaded condition. By assessing the values over a larger area $\left(1950 \mathrm{~mm}^{2}\right)$ than that of a single sensor $216 \mathrm{~mm}^{2}$ ), the PPI parameter provides a more reliable indicator of the pressure distribution behaviour of support surfaces than peak pressures alone ${ }^{42}$. Pressures were analysed for the areas covered by the Sebutapes, as well as for the sacral location with the highest pressure, regardless of the location on the buttocks (top left, top right, bottom left, bottom right).

As a clinical measure of tissue response, redness of the skin was scored after every 20-minute period, and recorded as absent, diffuse or clearly defined, and further categorised in terms of blanchable or non-blanchable. Redness of the skin was assessed independently by two of the authors (BH and PB) blinded for the spineboard allocation. Inter-rater reliability was tested using Cohen's kappa.

\section{Statistical analysis}

Statistical analysis was performed using the Statistical Package for Social Sciences (SPSS, IBM), version 20.0.0.1. Pressures are presented as mean \pm SD for each 20-minute time period and for each Sebutape location; lactate concentrations and IL1 $\alpha /$ TP ratio are presented as mean \pm SD for each 20 -minute time period; redness scores are presented in categories for each 20-minute time period. Statistical analysis of tissue-interface pressures, IL1 $\alpha$ /TP ratio and lactate concentrations was performed 
using repeated-measures analysis of variance with correction of the degrees of freedom using the Greenhouse-Geisser estimates of sphericity when Mauchly's test indicated that the assumption of sphericity had been violated. The analysis used time as the within-group factor and type of spineboard as the between-group factor. Posthoc group comparisons at the different time points were only performed when the overall repeated-measures tests were statistically significant. All scores were tested for normality using the Kolmogorov-Smirnov test. Parametric variables were compared using Student's t-test, while non-parametric variables were compared using the Mann-Whitney $U$ statistic, Wilcoxon signed-rank test and Spearman rank correlation. Nominal variables were compared across independent groups using the chi-squared test or Fisher's exact test. Homogeneity of variance was assessed using Levene's test. Level of significance was set at $P<0.05$.

A

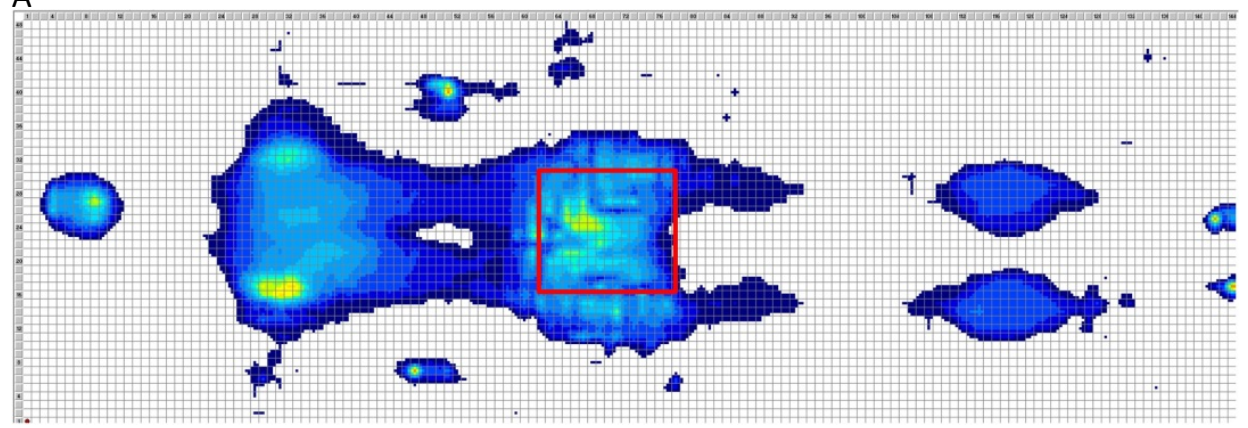

B

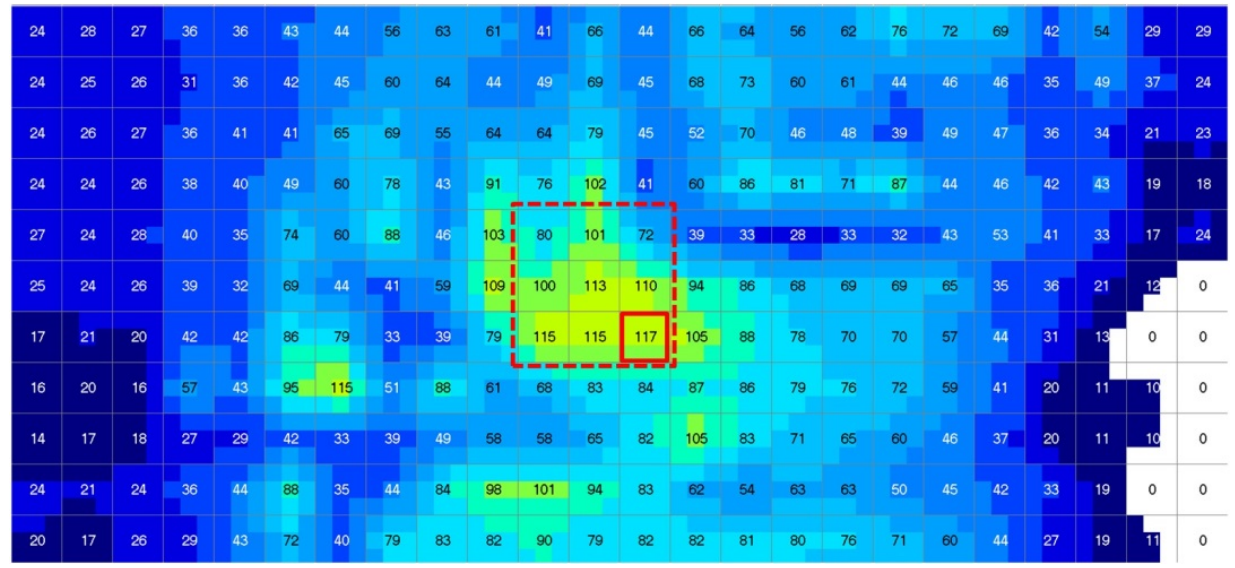

Figure 5.2 (A) Sample of pressure measurement; red square outlines sacral area. (B) Zoomed-in picture of pressures measured in sacral area; sensor with solid red line indicates sensor with Peak Pressure (in this case: $\mathrm{PP}=117 \mathrm{mmHg}$ ); area of $3 \times 3$ sensors indicates area for Peak Pressure Index calculation (in this case: $\mathrm{PPI}=102.6 \mathrm{mmHg}$ ). 


\section{Results}

\section{Tissue-interface pressures}

Both mean peak tissue-interface pressures and peak pressure indexes were higher on the rigid spineboard than on the soft-layered spineboard, for the majority of locations and time points $(P s<0.05$; Tables 5.1 and 5.2).

Table 5.1 Mean \pm standard deviation peak pressure and Peak Pressure Index in $\mathrm{mmHg}$ for the rigid spineboard, in relation to location and duration of loading

\begin{tabular}{lcccccc}
\hline Rigid spineboard & \multicolumn{2}{c}{ Peak pressure } & \multicolumn{4}{c}{ Peak pressure index } \\
\hline Location & $\begin{array}{c}20 \text { mins } \\
\text { loaded }\end{array}$ & $\begin{array}{c}40 \text { mins } \\
\text { loaded }\end{array}$ & $\begin{array}{c}60 \text { mins } \\
\text { loaded }\end{array}$ & $\begin{array}{c}20 \text { mins } \\
\text { loaded }\end{array}$ & $\begin{array}{c}40 \text { mins } \\
\text { loaded }\end{array}$ & $\begin{array}{c}60 \text { mins } \\
\text { loaded }\end{array}$ \\
\hline Top left & $215 \pm 144$ & $208 \pm 134$ & $251 \pm 157$ & $234 \pm 128$ & $212 \pm 126$ & $252 \pm 138$ \\
Bottom left & $138 \pm 62$ & $163 \pm 135$ & $127 \pm 76$ & $191 \pm 96$ & $202 \pm 106$ & $180 \pm 84$ \\
Top right & $78 \pm 89$ & $84 \pm 48$ & $138 \pm 93$ & $105 \pm 82$ & $102 \pm 53$ & $161 \pm 91$ \\
Bottom right & $117 \pm 98$ & $78 \pm 46$ & $93 \pm 73$ & $144 \pm 99$ & $117 \pm 64$ & $144 \pm 71$ \\
Max sacrum & $337 \pm 110$ & $314 \pm 122$ & $344 \pm 124$ & $284 \pm 104$ & $254 \pm 102$ & $282 \pm 117$ \\
\hline
\end{tabular}

Table 5.2 Mean \pm standard deviation peak pressure and Peak Pressure Index in $\mathrm{mmHg}$ for the softlayered spineboard, in relation to location and duration of loading

\begin{tabular}{lllllll}
\hline $\begin{array}{l}\text { Soft-layered } \\
\text { spineboard }\end{array}$ & \multicolumn{3}{c}{ Peak pressure } & \multicolumn{3}{l}{ Peak pressure index } \\
\hline Location & $\begin{array}{l}20 \mathrm{mins} \\
\text { loaded }\end{array}$ & $\begin{array}{l}40 \text { mins } \\
\text { loaded }\end{array}$ & $\begin{array}{l}60 \text { mins } \\
\text { loaded }\end{array}$ & $\begin{array}{l}20 \text { mins } \\
\text { loaded }\end{array}$ & $\begin{array}{c}40 \text { mins } \\
\text { loaded }\end{array}$ & $\begin{array}{c}60 \text { mins } \\
\text { loaded }\end{array}$ \\
\hline Top left & $28 \pm 11$ & $23 \pm 5$ & $28 \pm 11$ & $34 \pm 10$ & $30 \pm 7$ & $35 \pm 8$ \\
Bottom left & $35 \pm 8$ & $34 \pm 10$ & $34 \pm 10$ & $39 \pm 11$ & $36 \pm 10$ & $38 \pm 7$ \\
Top right & $33 \pm 8$ & $34 \pm 25$ & $27 \pm 10$ & $35 \pm 6$ & $34 \pm 9$ & $31 \pm 5$ \\
Bottom right & $32 \pm 10$ & $36 \pm 8$ & $37 \pm 10$ & $35 \pm 7$ & $37 \pm 5$ & $37 \pm 7$ \\
Max sacrum & $66 \pm 15$ & $66 \pm 15$ & $62 \pm 12$ & $42 \pm 9$ & $45 \pm 9$ & $44 \pm 7$ \\
\hline
\end{tabular}

On the rigid spineboard, mean tissue-interface pressures and pressure indexes differed significantly between the Sebutape locations at all time point $(P<0.05)$, but remained constant over time. On the soft-layered spineboard, pressures and pressures indexes were similar across locations and time points ( $P>0.05$ in all cases). On the rigid spineboard, mean tissue-interface pressures and pressure indexes at the sacral area were higher than those to the right of the sacrum $(P<0.05)$. On the softlayered spineboard, maximum pressures at the sacrum were higher than those measured at all four Sebutape locations $(P<0.01)^{1}$.

\footnotetext{
${ }^{1}$ All tests performed using Friedmans ANOVA for non-parametric related data
} 


\section{IL1 $\alpha$ /TP ratio}

The IL1 $\alpha$ /TP ratio showed a similar pattern over time on both the soft-layered and the rigid spineboard, for both the three loading and subsequent unloading periods (Figure 5.3; Table S5.1 (Appendix 5.1)). On both spineboards, the ratio increased significantly between values at the baseline and the first loading period $(P<0.01)$ and between the first and second loaded periods $(P<0.05)$. The decrease in the ratio after the first period of unloading was also significant $(P<0.05)$ for both spineboards. For the rigid spineboard, a significant decrease in IL1 $\alpha$ /TP ratio was also seen between the first and second unloaded periods $(P<0.05)$. Except for 40 -minutes unloaded period, there were no significant differences in IL1 $\alpha$ /TP ratio between the two spineboard for the various loaded and unloaded periods $(P \mathrm{~S}>0.05){ }^{2}$

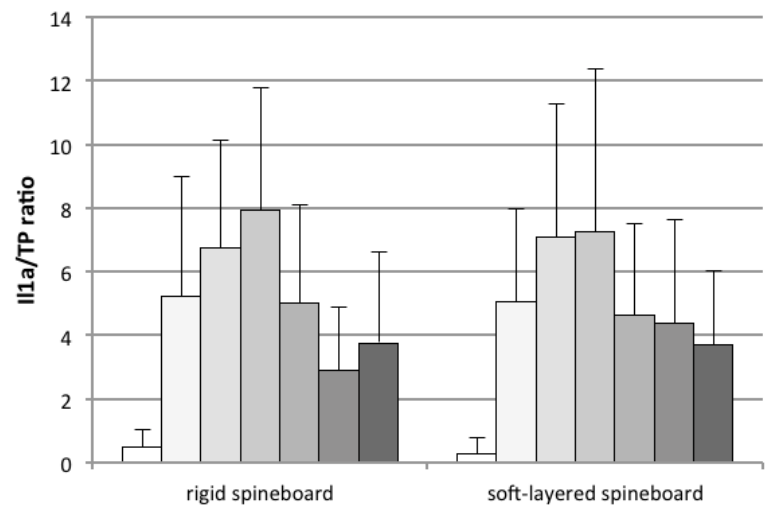

$\square$ baseline

$\square 20$ mins loaded

$\square 40$ mins loaded

$\square 60$ mins loaded

$\square 20$ mins unloaded

$\square 40$ mins unloaded

$\square 60$ mins unloaded

Figure 5.3 IL1 $\alpha$ /TP ratio, average of the four locations, comparing the two devices over time. Presented data are means + standard deviation

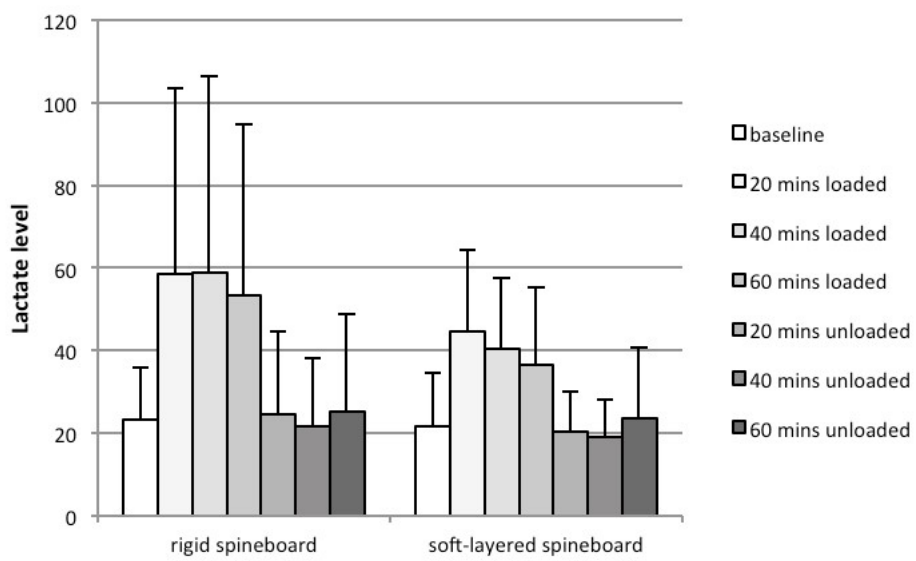

Figure 5.4 Lactate levels, average of the four locations, comparing the two devices over time. Presented data are means + standard deviation

\footnotetext{
${ }^{2}$ All tests performed using paired samples t-test
} 


\section{Lactate}

Figure 5.4 illustrates the corresponding lactate concentrations for each of the loading/unloading periods for both spineboards (see also Appendix 5.1, Table S5.1) . These values were clearly higher during the loaded periods compared to the corresponding baseline values. Indeed lactate concentrations increased significantly for both spineboards for the first loaded period $(P<0.05)$ and decreased significantly for the first unloaded period when compared to the value after 60 minutes of loading (Ps<0.01). Lactate concentrations remained stable over both the loaded period and the unloaded period. No differences between the two spineboards were seen in lactate levels for the various periods $(P>0.05) .^{3}$

\section{Skin redness}

The results of the redness categorisation is shown in Figure 5.5. Log-linear regression analysis revealed a significant difference between the two spineboards in both the intensity and course of pressure-induced redness. In particular, the soft-layered spineboard generally caused no or only diffuse redness after several periods of loading. By contrast, the rigid spineboard produced clearly defined redness even after the first period of loading $\left(\chi^{2}(1)=23.24, P<0.01\right)$.

Complete agreement between the two observers with regards to level of redness was found in $77 \%$ of the cases, resulting in a kappa value of 0.61 . Disagreement was mostly seen in the assessment of no redness versus diffuse redness (13\%). All redness was blanchable.

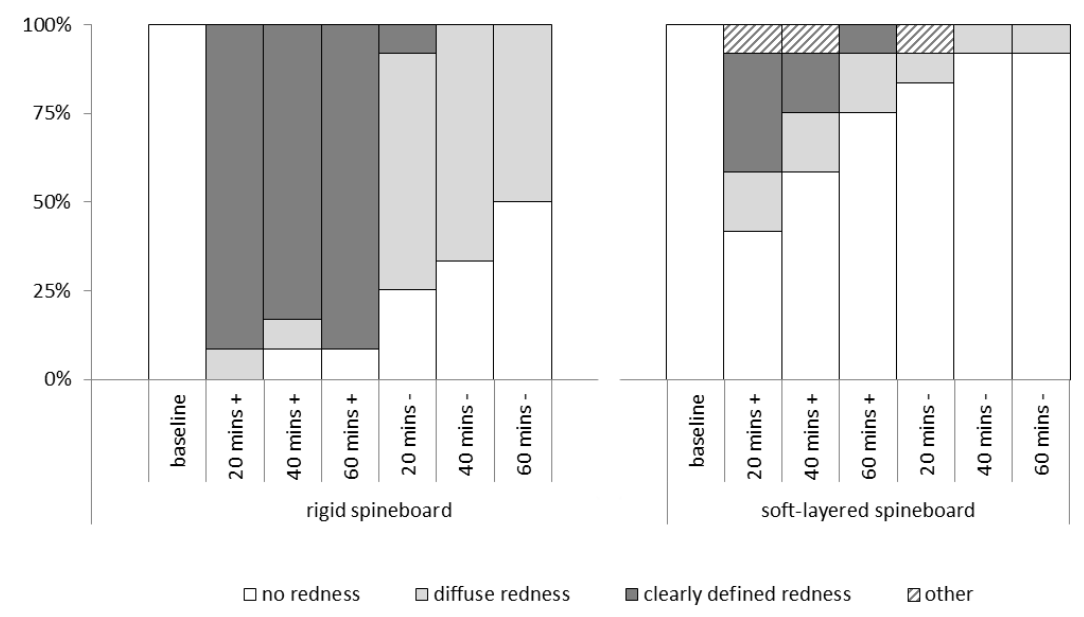

Figure 5.5 Redness of the buttocks in relation to time point and device +: loaded: -: unloaded. Other: red streak within the intergluteal cleft, no redness on the buttock

\footnotetext{
${ }^{3}$ All tests performed using Wilcoxon matched-pairs test
} 


\section{Interactions between variables}

Table 5.3 shows the interactions (or lack thereof) found between tissue-interface pressure and skin redness, IL1 $\alpha /$ TP ratio and lactate.

A significant relation was found between tissue-interface pressure and skin redness, with higher pressures correlating with higher levels of skin redness.

There was no significant correlation between tissue-interface pressures and IL1 $\alpha$ /TP ratio for either the rigid or the soft-layered spineboard. No significant relation between pressure and lactate level was seen for the soft-layered spineboard but there was a significant relation between pressure and lactate levels for the rigid spineboard. For both the rigid spineboard and the soft-layered spineboard, a significant correlation was found between redness and IL1 $\alpha$ /TP ratio, and between redness and lactate levels.

Table 5.3 Correlations between tissue-interface pressure and skin redness, IL1 $\alpha /$ TP ratio and lactate

\begin{tabular}{lcccc}
\hline & $\begin{array}{c}\text { Tissue-interface } \\
\text { pressure }\end{array}$ & Skin redness & IL1 $\alpha /$ TP ratio & Lactate \\
\hline Tissue-interface & - & $R=0.707, P<0.01$ & $R=0.017, P=1.0$ (RSB); & $\mathrm{R}=0.2, P<0.05$ (RSB); \\
pressure & & $R=0.070 ; P=0.4$ (SLSB) & $R<0.01, P=1.0$ (SLSB) \\
Skin redness & & $R=0.634, P<0.01$ (RSB); & $R r=0.264, P<0.05$ (RSB); \\
& & $R=0.286, P<0.05$ (SLSB) & $R=0.243, P<0.05$ (SLSB) \\
IL1 $\alpha /$ TP ratio & & - & n.a. \\
Lactate & & & - \\
\hline
\end{tabular}

* RSB: rigid spineboard; SLSB: soft-layered spineboard; n.a. not applicable

\section{Discussion}

This prospective, randomised open label crossover study examined the relationship between tissue-interface pressure, and the response of the skin in healthy participants lying supine on two types of spineboards. More specifically, we tested the hypothesis that lying on a rigid spineboard would result in an elevated release of IL1 $\alpha$ and lactate as a result of the increased tissue-interface pressures when compared to lying on a soft-layered spineboard. In addition, we hypothesised that there would be a relationship between the pressure-induced reactive hyperaemia and the ILI $\alpha$ and lactate concentrations. We found marked differences between the effects of the rigid spineboard and the soft-layered spineboard with regard to tissue-interface pressure and redness of the skin. In addition, IL1 $\alpha$ /TP ratios increased during the loaded period on the spineboards and remained elevated during the unloaded period when compared to the baseline measurements. By contrast, the elevated lactate concentrations remained stable during the loaded period but were restored to 
baseline values levels within the first unloaded period. Furthermore, IL1 $\alpha$ /TP ratios and lactate concentrations, which were similar between the two spineboards, correlated with skin redness, but not with the magnitude of tissue-interface pressures.

To our knowledge, this is one of the first studies to relate IL1 $\alpha$ /TP ratio and lactate concentration to tissue-interface pressures in a clinically relevant scenario ${ }^{43}$. Earlier studies have applied pressures to engineered skin constructs ${ }^{34-36}$ and controlled mechanical loading of the forearm of healthy volunteers ${ }^{36}$. In accordance with our earlier study ${ }^{28}$, we found a clear relationship between tissue-interface pressure and redness of the skin on both types of spineboard. The traditional rigid spineboard showed mean tissue-interface pressures significantly higher than the corresponding values measured on the soft-layered spineboard. Pressures were measured across the entire sacral area, including the four sites where the Sebutapes were placed. These local pressures were recorded in order to identify any local correlations between pressures and IL1 $\alpha$ /TP ratio and lactate concentrations. In addition, we determined the peak pressure in the sacral area, to test for global effects of pressure. No clear correlations were found between either the local or the global tissue-interface pressures and IL1 $\alpha$ /TP ratio or lactate concentration. However, IL1 $\alpha /$ TP ratio revealed a significant increase during the first 40 minutes in the loaded conditions for both spineboards (Figure 5.2), but remained stable during the last 20 minute loaded period. Lactate remained stable over the three loaded periods for both spineboards (Figure 5.3). This reflects the nature of the response of these markers, where IL1 $\alpha$ release is part of a complex tissue regeneration process, while lactate concentration, a marker of anaerobic metabolism, is associated with the availability of oxygen and other nutrients within the tissues. By contrast, a clear relationship was found between the level of skin redness and both the IL1 $\alpha$ /TP ratios and lactate concentration. It appears that either IL1 $\alpha$ and lactate are released at a lower pressure threshold than the pressure needed to induce reactive hyperaemia, or that they are upregulated as an epiphenomenon. This uncertainty about the regulating mechanism underlying our findings may abnegate the clinical applicability of using these biomarkers for the assessment of pressure-related skin damage. Further research concerning whether abolishing the inflammatory response directly, reduces the risk of pressure-related skin damage are warranted.

A few remarks should be made about the methods used in this study. First, the study population is quite homogenous in terms of age, sex and ethnicity, and therefore probably not representative of the population at highest risk of pressure ulcer onset. Also, although tissue-interface pressures are an easy way to get a first indication of the effect of the surface on the body, they may not be accurate in predicting the onset of pressure ulcers ${ }^{30}$. Furthermore, we restricted the investigation to measuring IL1 $\alpha$ and lactate concentrations, as both have been implicated as biomarkers related to tissue damage. However, other markers may be more specific for the pressureinduced changes in skin physiology ${ }^{43}$. In addition, measurements in the loaded 
condition were performed in three periods of 20 minutes, with a short unloaded period of two minutes in between to replace the Sebutapes and photograph the buttock area. Although unlikely ${ }^{35}$, these brief reperfusion periods may have affected the local expressions of cytokines.

As shown in an earlier study from our group ${ }^{27-29}$, the rigid spineboard induces high levels of stresses and strains on the soft tissues, whereas these effects are significantly less on the soft-layered spineboard. The real danger of the rigid spineboard may therefore be to damage the deeper tissue layers, whereas the using soft-layered spineboard avoids the fast deformation damage ${ }^{29}$. Although IL1 $\alpha$ and lactate concentrations in the skin may not be the appropriate biomarkers to indicate deep tissue injury, other markers such muscular fatty-acid binding protein may be released in response to ischemic muscle damage $\mathrm{e}^{44-46}$, and could be used as indicators of this specific type of tissue damage. Finally, skin hyperaemia was assessed semiquantitatively. While other, more sensitive methods ${ }^{47}$, could have been used, the agreement between the two assessors was good.

\section{Conclusion}

Cutaneous IL1 $\alpha$ and lactate both have a strong relationship with pressure exposure time, but not with pressure magnitude. Furthermore, IL1 $\alpha$ was measured even in the absence of visible redness of the skin. The study may offer the potention of biomarkers, reflecting inflammation and/or tissue metabolism, for use in assessing the effects of prolonged spineboard support, but further studies are warranted. 


\section{Appendix 5.1}

Table S5.1 Mean IL1a/TP ratio and lactate levels and lactate levels for the rigid and soft-layered spineboard, in relation to duration of loading.

\begin{tabular}{llccccccc}
\hline & Baseline & $\begin{array}{c}20 \text { mins } \\
\text { loaded }\end{array}$ & $\begin{array}{c}40 \text { mins } \\
\text { loaded }\end{array}$ & $\begin{array}{c}60 \text { mins } \\
\text { loaded }\end{array}$ & $\begin{array}{c}20 \text { mins } \\
\text { unloaded }\end{array}$ & $\begin{array}{c}40 \text { mins } \\
\text { unloaded }\end{array}$ & $\begin{array}{c}60 \text { mins } \\
\text { unloaded }\end{array}$ \\
\hline $\begin{array}{l}\text { Rigid } \\
\text { spineboard }\end{array}$ & $\begin{array}{l}\text { IL1A/TP } \\
\text { ratio } \\
\text { Lactate } \\
\text { (mmol/I) }\end{array}$ & 0.48 & 5.24 & 6.73 & 7.91 & 5.00 & 2.91 & 3,75 \\
$\begin{array}{l}\text { Soft- layered } \\
\text { spinboard }\end{array}$ & $\begin{array}{l}\text { IL1A/TP } \\
\text { ratio } \\
\text { Lactate } \\
\text { (mmol/l) }\end{array}$ & 0.27 & 5.05 & 7.09 & 7.26 & 4.64 & 4.38 & 3.69 \\
& 21.7 & 44.5 & 40.5 & 36.6 & 20.3 & 19.0 & 23.6 \\
\hline
\end{tabular}




\section{References}

1. American College of Surgeons Committee on Trauma. Advanced Trauma Life Support for doctors, 2012: Chicago, IL.

2. National Association of Emergency Medical Technicians. Prehospital Trauma Life Support. 7th ed2010, Burlington, MA: Jones \& Bartlett Learning.

3. Ahn H, Singh J, Nathens A, et al. Pre-hospital care management of a potential spinal cord injured patient: a systematic review of the literature and evidence-based guidelines. J Neurotrauma. 2011;28: 1341-1361.

4. Vickery D. The use of the spinal board after the pre-hospital phase of trauma management. Emerg Med J. 2001;18:51-54.

5. Cooney DR, Wallus $\mathrm{H}$, Asaly $\mathrm{M}$, Wojcik S. Backboard time for patients receiving spinal immobilization by emergency medical services. Int J emerg Med. 2013;6:17-19.

6. Lerner EB, Moscati R. Duration of patient immobilization in the ED. Am J Emerg Med. 2000;18: 28-30.

7. Yeung JHH, Cheung NK, Graham CA, Rainer TH. Reduced time on the spinal board - effects of guidelines and education for emergency department staff. Injury. 2006;37:53-56.

8. Schouten R, Albert T, Kwon BK. The spine-injured patient: initial assessment and emergency treatment. J Am Acad Orthop Surg. 2012;20:336-346.

9. Kwan I, Bunn F. Effects of prehospital spinal immobilization: a systematic review of randomized trials on healthy subjects. Prehosp Disaster Medicine. 2005;20(1):47-53.

10. Gunes UY. A descriptive study of pressure ulcer pain. Ostomy Wound Manag. 2008;54:56-61.

11. Pieper B, Langemo D, Cuddigan J. Pressure ulcer pain: a systematic literature review and National Pressure Ulcer Advisory Panel white paper. Ostomy Wound Manag. 2009;55:16-31.

12. Rastinehad D. Pressure ulcer pain. J Wound Ostomy \& Continence Nursing, 2006. 33: p. 252-257.

13. Fox C. Living with a pressure ulcer: a descriptive study of patients' experiences. Br J Community Nurs. 2002;7:11-22.

14. Hopkins A, Dealey C, Bale S, DeFloor T, Worboys F. Patient stories of living with a pressure ulcer. J Adv Nurs. 2006:345-353.

15. Bennett G, Dealey C, Posnett J. The cost of pressure ulcers in the UK. Age Ageing. 2004;33:230-235.

16. Chapman BR, Mills KJ, Pearce LM, Crowe TC. Use of an arginine-enriched oral nutrition supplement in the healing of pressure ulcers in patients with spinal cord injuries:n an observational study. Nutrition Dietetics. 2011;68:208-213.

17. Sanada $\mathrm{H}$, lizaka $\mathrm{S}$, Matsui $\mathrm{Y}$, et al. Clinical wound assessment using DESIGN-R total score can predict pressure ulcer healing: pooled analysis from two multicenter cohort studies. Wound Repair Regen. 2011;19:559-567.

18. Allman RM, D.A.S.M. Pressure ulcers, hospital complications, and disease severity: impact on hospital costs and length of stay. Adv Skin Wound Care. 1999;12:22-30.

19. Graves N, Birrell FA, Whitby M. Modeling the economic losses from pressure ulcers among hospitalized patients in Australia. Wound Repair Regen, 2005;13:465-467.

20. Essex HN, Clark M, Sims J, Warriner A, Cullum N. Health-related quality of life in hospital inpatients with pressure ulceration: assessment using generic health-related quality of life measures. Wound Repair Regen. 2009;17:797-805.

21. Gorecki C, Brown JM, Nelson EA, et al. Impact of pressure ulcers on quality of life in older patients: a systematic review. J Am Geriatr Soc. 2009;57:1175-1183.

22. Langemo DK, Melland $H$, Hanson D, Olson B, Hunter $S$. The lived experience of having a pressure ulcer: a qualitative analysis. Adv Skin Wound Care. 2000;13:225-235.

23. Spilsbury K, Nelson A, Cullum N, Iglesias C, Nixon J, Mason S. Pressure ulcers and their treatment and effects on quality of life: hospital inpatient perspectives. J Adv Nurs. 2007;57:494-504.

24. DeFloor T. The risk of pressure sores: a conceptual scheme. J Clin Nurs. 1999;8:206-216.

25. Luscombe MD, Williams JL. Comparison of a long spinal board and vacuum mattress for spinal immobilisation. Emerg Med J. 2003;20:476-478.

26. Mak AFT, Yu Y, Kwan LPC, Sun L, Tam EWC. Deformation and reperfusion damages and their accumulation in subcutaneous tissues during loading and unloading: a theoretical modeling of deep tissue injuries. J Theoretical Biology. 2011;11:65-73. 
27. Hemmes B, Poeze M, Brink PRG. Reduced tissue-interface pressure and increased comfort on a newly developed soft-layered long spineboard. J Trauma. 2010;68:593-598.

28. Hemmes B, Brink PRG, Poeze $M$. Effects of unconsciousness during spinal immobilization on tissueinterface pressures: A randomized controlled trial comparing a standard rigid spineboard with a newly developed soft-layered long spineboard. Injury. 2014;45:1741-1746.

29. Oomens CWJ, Zenhorst W, Broek M, et al. A numerical study to analyse the risk for pressure ulcer development on a spine board. Clin Biomech. 2013;28:736-742.

30. Linder-Ganz E, Shabshinb N, Itzchak Y, Gefen A. Assessment of mechanical conditions in sub-dermal tissues during sitting: a combined experimental-MRI and finite element approach. J Biomech. 2007; 40:1443-1454.

31. Sopher R, Nixon J, Gorecki C, Gefen A. Exposure to internal muscle tissue loads under the ishial tuberosities during sitting is elevated at abnormally high or low body mass indices. J Biomech., 2010; 43:280-286.

32. Loerakker S, Manders E, Strijkers GJ, et al. The effects of deformation, ischemia, and reperfusion on the development of muscle damage during prolonged loading. J Appl Physiol. 2011;111:1168-1177.

33. Oomens CWJ, Loerakker S, Bader DL. The importance of internal strains as opposed to interface pressures in the prevention of pressure related deep tissue injury. J Tissue Viability. 2010;19:35-42.

34. Bronneberg D, Bouten CVC, Oomens CWJ, van Kemenade PM, Baaijens FPT. An in vitro model system to study the damaging effects of epidermal loading. Ann Biomech Eng. 2006;34:506-514.

35. Cornelissen LH, Bronneberg D, Bader DL, Baaijens FPT, Oomens CWJ. The transport profile of cytokines in epidermal equivalents subjected to mechanical oading. Ann Biomech Eng. 2009;37: 1007-1018.

36. Bronneberg D. Biochemical markers for early detection of superficial pressure ulcers; Ch. 6, 2007, Eindhoven University of Technology, Dept. of Biomedical Technology: Eindhoven, the Netherlands.

37. Lans van der M. Biochemical marker for early and objective detection of grade 1 pressure ulcers; MSc thesis, 2007, Eindhoven University of Techonology: Eindhoven, the Netherlands.

38. Martaheri P, Gjovaag T, Worsley PR, Bader DL. A review of the role of the partial pressure of carbon dioxide in mechanically loaded tissues: the canary in the cage singing in tune with the pressure ulcer mantra. Ann Biomech Engineering. 2015;43:336-367.

39. Bronneberg D, Spiekstra SW, Cornelissen LH, Oomens CWJ, Gibss S, Baaijens FPT, et al. Cytokine and chemokine release upon prolonged mechanical loading of the epidermis. Exper Dermatol. 2007;16: 567-573.

40. Perkins MA, Osterhues MA, Farage MA, Robinson MK. A noninvasive method to assess skin irritation and compromised skin conditions using simple tape adsorption of molecular markers of inflammation. Skin Research and Technology. 2001;7:227-237.

41. Julious SA. Sample size of 12 per group rule of thumb for a pilot study. Pharm Stat. 2005;4:287-298.

42. Keller BPJA, van Overbeeke J, van der Werken Chr. Interface pressure measurement during surgery: a comparison of four operating table surfaces. J Wound Care. 2005;15:5-9.

43. Worsley PR, Prudden G, Gower G, Bader DL. Investigating the effects of strap tension during noninvasive ventilation mask application: a combined biomechanical and biomarker approach. Medical Devices: Evidence and Research. 2016;9:409-417.

44. Glatz JFC, Kleine AH, van Nieuwenhoven FA, et al. Fatty-acid-binding protein as a plasma marker for the estimation of myocardial infarct size in humans. Br Heart J. 1994;71:135-140.

45. Knight SL, Taylor RP, Polliack A, Bader DL. Establishing predictive indicators for the status of loaded soft tissues. J Appl Physiol. 2001;90:2231-2237.

46. Pelsers MMAL, Hernems WT, GLatz JFC. Fatty acid-binding proteins as plasma markers of tissue injury. Clinica Chimica Acta. 2005;352:15-35.

47. Humeau-Heurtier A, Guerreschi E, Abraham P, Mahé G. Relevance of laser Doppler and laser Speckle techniques for assessing vascular function: state of the art and future trends. IEEE T Bio-Med Eng. 2013;60:659-666. 


\section{Chapter}

\section{Improving the effect of shear on skin viability with wound dressings}

L.A. de Wert, L. Schoonhoven, J.H.C.H. Stegen, A.A. Piatkowski, R.R. van der Hulst, M. Poeze, N.D. Bouvy Journal of the mechanical behaviour of biomedical materials, 2016;60:505-514 


\section{Abstract}

\section{Background}

Pressure ulcers are a major healthcare problem and caused by pressure and shear-forces. Although shear-force is understood to be a major contributing factor, no preventive interventions are specifically aimed at relieving the effect of shear on skin to improve skin viability.

\section{Methods}

A physical model was used to apply a combined loading of $2.4 \mathrm{kPa}$ pressure and $14.5 \mathrm{~N}$ shearforce on skin in humans. Loading was applied on the volar aspect of both forearms for 30 minutes in ten healthy volunteers. One arm received loading on skin with a wound dressing, the other arm (control) received loading directly on skin. The following parameters were determined before and after loading: IL-1 $\alpha /$ Total Protein-ratio (used as a measure of skin damage); Cutaneous blood cell flux ((CBF) measure of reactive hyperaemia); Lactate concentration (measure of tissue ischemia).

Three different dressings were tested on three different days. The order of dressing application, dressing arm and start of the intervention were randomized.

\section{Results}

Participants mean (SEM) age was $22.5 \pm 1.6$ year with a BMI of $22.3 \pm 2.4 \mathrm{~kg} / \mathrm{m}^{2}$. IL- $1 \alpha /$ Total Protein-ratio of the skin was significantly lower after the application of pressure and shear when the Mepilex ${ }^{\circledR}(P<0.01)$, Allevyn $(P<0.05)$ or Aquacel ${ }^{T M}$ dressing $(P<0.01)$ was used compared with the control measurement. The Mepilex ${ }^{\circledast}$ dressing was more effective in reducing post-load IL-1 $\alpha /$ Total Protein- ratio compared to the Allevyn dressing $(P<0.01)$.

Post- load CBF was significantly lower when the Mepilex ${ }^{\circledast}$ or Aquacel dressing was used $(P<0.001)$. Both dressings induced significantly less post- load $C B F$ than the Allevyn dressing $(P<0.01$ and $P<0.001$, respectively).

The concentration of lactate was not significantly increased after the application of pressure and shear and could not be used as a measure with this model.

\section{Conclusion}

This is the first in vivo study to demonstrate that the effects of pressure in combination with shear on skin viability can be improved with foam dressings. In this study, the multi- layered dressings perform better than the single- layered dressing. 


\section{Introduction}

A pressure ulcer occurs as a result of prolonged mechanical loading of the skin and underlying tissue. Persons who are bound to a bed or (wheel)chair are at particularly high risk, as pressure ulcers are most likely to develop in areas that are exposed to pressure or pressure in combination with shear, usually at the interface of a supporting surface and the skin over a bony prominence (EPUAP/NPUAP Guidelines 2014). With prevalence rates reported up to $12 \%$ in hospitals ${ }^{1}$, adverse health outcomes and high treatment costs ${ }^{2,3}$, it remains a frequent and significant problem. As it is seen as an important quality indicator as well ${ }^{4}$, many efforts are made to reduce the development of pressure ulcers.

Traditionally, pressure ulcer preventive strategies include periodic repositioning ${ }^{5}$ and reduction of pressure. Although shear is seen as an important contributory factor as well $^{6-10}$, preventive interventions are particularly aimed at pressure redistribution and many pressure relieving devices have been developed to accomplish this ${ }^{11}$.

Shear forces are forces that acts parallel on an area of the skin at the interface of a support surface, such that there will be deformation of tissue in parallel and perpendicular planes. Shear forces can become quite high (up to $40 \mathrm{~N}$ ) in patients, especially at the sacral area when the head of the patient's bed is elevated ${ }^{12,13}$.

To prevent the development of pressure ulcers, the prophylactic application of foam dressings is recommended to areas of the skin exposed to shear, such as the sacral area or heels ${ }^{14}$. These dressings can possibly relieve the harmful effects of shear on the skin and protects the skin against breakdown. However, this is only supported by ex vivo laboratory test results ${ }^{15-17}$. Therefore, it remains unclear if foam dressings can actually improve skin viability by reducing the effect of shear on the skin in humans and if so, what type of foam dressing performs best.

In a previous study ${ }^{18}$ we described a novel method which enables us to evaluate the effects of both pressure and shear in healthy volunteers. After a combined loading of both pressure and shear for 30 minutes on the skin in humans, a higher increase in IL$1 \alpha /$ Total protein-ratio and reactive hyperaemia was measured compared to the response of applied pressure alone. Consequently, this method provides us the opportunity to evaluate foam dressings aimed at relieving the effect of shear on skin in vivo.

Therefore, the aim of this study was to investigate if wound dressings are capable of improving the effect of shear on skin viability in humans. Furthermore, we wanted to investigate if multi-layered foam dressings perform better than a single layered foam dressing. To accomplish this, we investigated the response of the skin in healthy participants after a combined loading of pressure and shear in the absence or presence of three commercially available foam dressings. 


\section{Methods}

\section{Ethical considerations}

The study was approved by the medical ethics committee of the Maastricht University medical Centre. The research protocol was registered in the clinicaltrials.gov database as number NCT02348671. The study was conducted in compliance with ethical rules for human experimentation that are stated in the Declaration of Helsinki and monitored according to the principles of Good Clinical Practice. Written informed consent was obtained of all participants.

\section{Physical model}

A sphysical model was used as previously described ${ }^{18}$. Briefly, this model included an identer: an anti-slipmat (Digibuddy, Onni-tec $\mathrm{GmbH}$, Germany) of 50 X $50 \mathrm{~mm}$, which was placed on the volar aspect of the forearm and loaded with a $1 \mathrm{~kg}$ lead. A $1.7 \mathrm{~kg}$ mass was connected with a line to produce shear on skin. Due to friction in the pulley and an angle of $15^{\circ}$, the actual shear was $14.5 \mathrm{~N}$ (measured with a spring balance) with a pressure of $2.4 \mathrm{kPa}$. A picture of the physical model is presented in Figure 6.1.

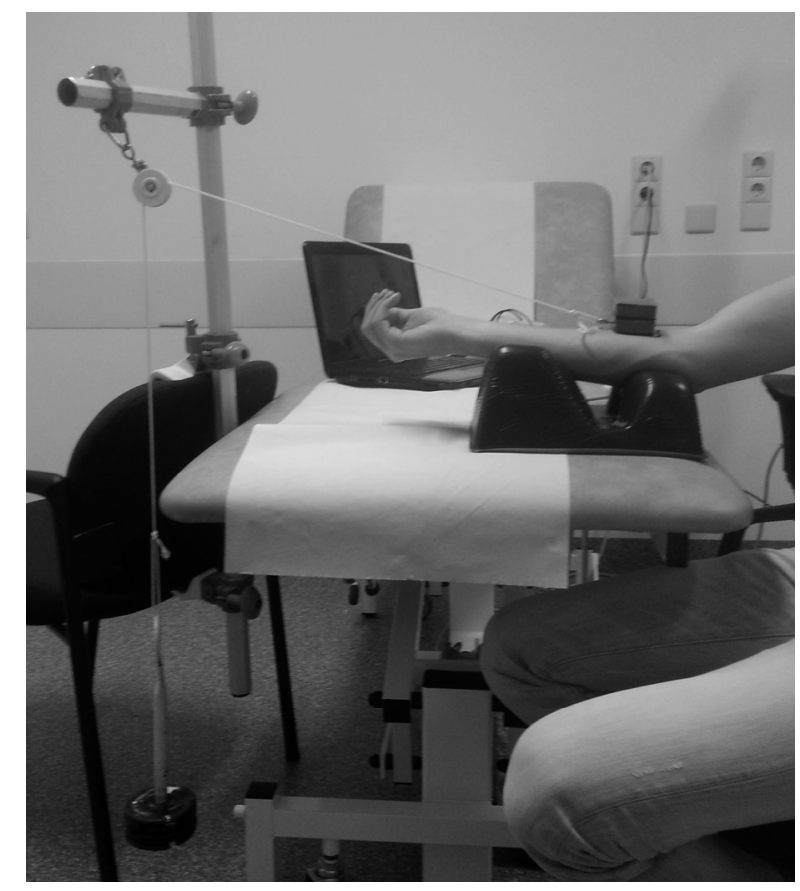

Figure 6.1 Physical model. An antislip mat $(50 \mathrm{~mm} \times 50 \mathrm{~mm})$ was placed at the volar aspect of the forearm and exposed to a $1 \mathrm{~kg}$ lead. To ensure stability of the lead weight, the line was set at a $15^{\circ}$ angle. 


\section{Participants and procedures}

Inclusion criteria were: healthy male volunteer, age between 20-30 years and a Body Mass Index (BMI) ranged between $20-30 \mathrm{~kg} / \mathrm{m}^{2}$. Participants were not able to participate if they had any active skin diseases. In addition, other exclusion criteria were: Diabetes Mellitus, trauma at the volar aspect of their forearms, muscular dystrophy, malignancy and non- steroidal anti- inflammatory drugs intake the last seven days. Participants were not allowed to drink caffeine/ alcohol or smoke before the experiment on a test day.

Three commercially available wound dressings were tested (Figure 6.2 and Table 6.1) in all participants on three separate test days. Age, height, weight, BMI, and blood pressure of all participants were recorded.

Table 6.1 Dressings used in this study

\begin{tabular}{|c|c|c|c|c|c|c|}
\hline Brand name & Materials & $\begin{array}{l}\text { Foam } \\
\text { layers }\end{array}$ & $\begin{array}{l}\text { Skin contact } \\
\text { layer }\end{array}$ & Size & Thickness & Manufacturer \\
\hline Mepilex ${ }^{\circledR}$ Border & $\begin{array}{l}\text { Polyurethane foam, } \\
\text { non- woven spreading } \\
\text { layer, polyacrylate } \\
\text { fibres }\end{array}$ & 3 & $\begin{array}{l}\text { Silicon } \\
\text { adhesive }\end{array}$ & $\begin{array}{l}10 \mathrm{~cm} \times 10 \\
\mathrm{~cm}\end{array}$ & $4 \mathrm{~mm}$ & $\begin{array}{l}\text { Mölnlycke } \\
\text { Healthcare } \\
\text { AB, } \\
\text { Göteborg, } \\
\text { Sweden }\end{array}$ \\
\hline Allevyn Adhesive & Hydrocellular foam & 1 & $\begin{array}{l}\text { Silicon } \\
\text { adhesive }\end{array}$ & $\begin{array}{l}10 \mathrm{~cm} \times 10 \\
\mathrm{~cm}\end{array}$ & $4 \mathrm{~mm}$ & $\begin{array}{l}\text { Smith \& } \\
\text { Nephew, } \\
\text { London, } \\
\text { England }\end{array}$ \\
\hline Aquacel $^{\mathrm{TM}}$ Foam & $\begin{array}{l}\text { Polyurethane foam, } \\
\text { hydrofiber }\end{array}$ & 2 & $\begin{array}{l}\text { Border: silicon } \\
\text { adhesive, } \\
\text { centre: } \\
\text { hydrofiber }\end{array}$ & $\begin{array}{l}10 \mathrm{~cm} \times 10 \\
\mathrm{~cm}\end{array}$ & $4 \mathrm{~mm}$ & $\begin{array}{l}\text { Convatec inc, } \\
\text { Skillman, USA }\end{array}$ \\
\hline
\end{tabular}

On a test day, the participants were asked to relax both their arms on a support cushion for a acclimatisation period of 20 minutes at room temperature $\left(21^{\circ} \mathrm{C} \pm 0.4\right)$. The borders of a $50 \mathrm{~mm} \times 50 \mathrm{~mm}$ area (equivalent to the area of the shear pad) on the volar aspect of both forearms at $2 \mathrm{~cm}$ of the centre of the cubital fossa were marked with a permanent marker. Baseline measurements of the unloaded skin of both forearms (dressing arm and control arm) were performed, incorporating: collection of IL-1 $\alpha$, Total Protein and lactate acid from the skin with the placement of a Sebutape sample (Cuderm Corp, Dallas, TX) in the centre of the marked area for two minutes, and cutaneous blood cell flux measurement performed with a Laser Doppler imaging system (MoorLDI2- Burn Imager, Moor Instruments Ltd, Axminster, United Kingdom). 
A
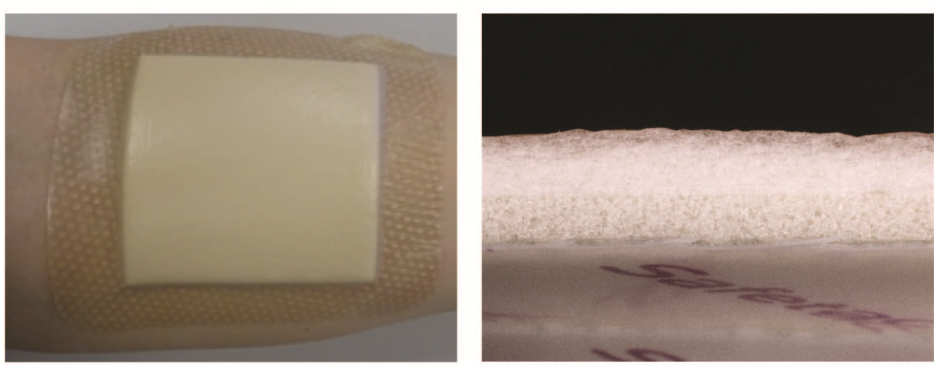

B
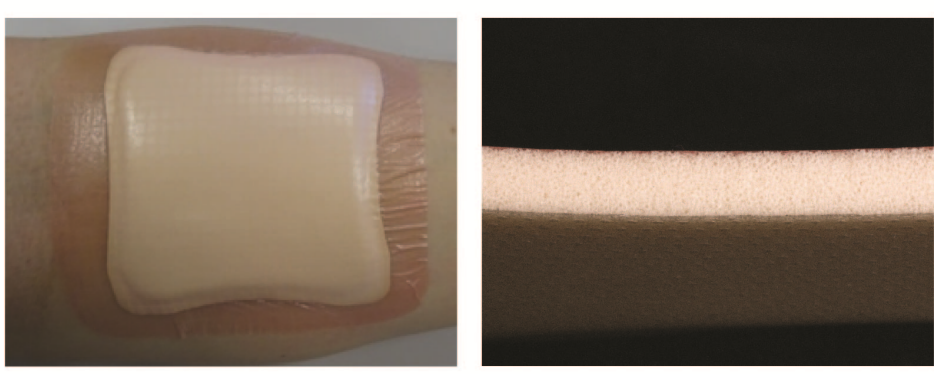

C
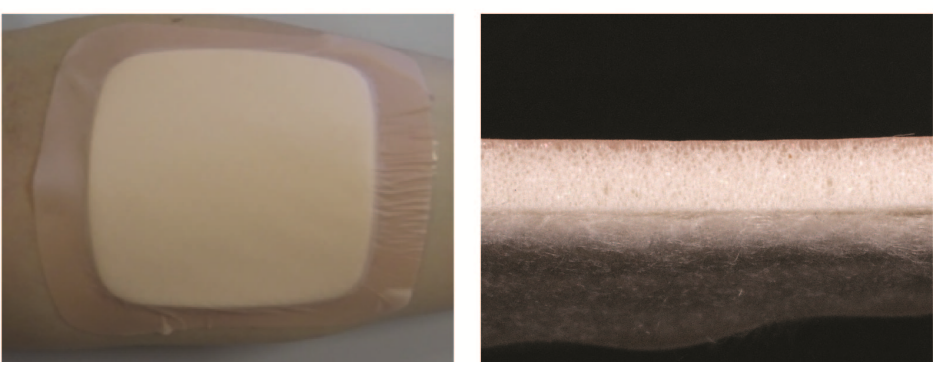

Figure 6.2 Photographs of the dressings and cross-sectional views through the dressing. $A=M^{2}$ Milex $^{\circledR}$ Border dressing, $\mathrm{B}=$ Allevyn Adhesive dressing, $\mathrm{C}=$ Aquacel $^{\mathrm{T} \mathrm{M}}$ Foam dressing.

We used the physical model as previously described. The identer of the shear model was placed over the marked area to apply a combined loading of $2.4 \mathrm{kPa}$ in combination with $14.5 \mathrm{~N}$ shear on the skin. One arm received loading on the skin with a dressing between the shear model and the skin (dressing arm). The other arm served as a control and received loading directly on the skin (control arm). After 30 minutes of loading, the measurements with the Sebutape and Laser Doppler were repeated and these represent the post- load measurements (see Figure 6.3). Every Sebutape was placed in a vial and stored in a $-80^{\circ} \mathrm{C}$ freezer until further processing.

The test protocol was repeated at two other test days in every participant to investigate the other dressings. For every participant, at least 24 hours was between each test day to make sure that cutaneous blood flow and IL-1 $\alpha$ concentrations returned to normal baseline levels before the test protocol was repeated. 
The order of dressing application, the site of dressing application (left or right arm) and the start of the intervention with or without dressing (control measurement) were randomized for each participant.

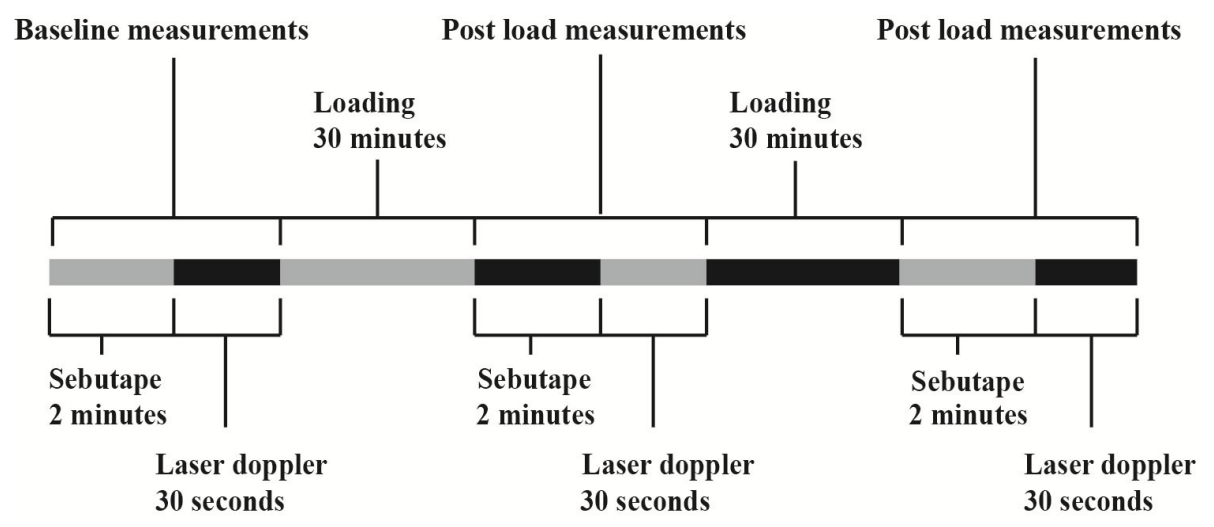

Figure 6.3 Experimental design. Baseline measurements were performed at the same time on both arms. Loading was applied on the skin of the dressing arm or control arm followed by a postload measurement with the Sebutape and Laser Doppler. Then loading was applied on the skin of the other arm (dressing arm or control arm) followed by a post- load measurement with the Sebutape and Laser Doppler. Dressing arm (left or right) and start of the intervention (with or without dressing) were randomized.

\section{Biochemical analysis}

A Sebutape sample, which represents a small adhesive patch, was used to absorb proteins and lactate from the skin in a non-invasive manner. Two millilitre $(\mathrm{ml})$ of Phosphate Buffered Saline was added to each Sebutape sample and then sonicated for ten minutes and vortexed for a further two minutes. The extraction of proteins and metabolites from the Sebutape was based on a study by Perkins et al. ${ }^{19}$.

Lactate concentration in the samples was enzymatically analysed using the Cobas Fara Centrifugal Spectrophotometer (Roche Diagnostica, Basel, Switzerland).

IL-1 $\alpha$ concentration in the samples was measured by a commercially available human IL-1 $\alpha$ / IL1F1 enzyme-linked immunosorbent assay (ELISA)kit (DuoSet R\&D system) with a detection range of $3.9-250 \mathrm{pg} / \mathrm{ml}$. To correct for differences in sebum uptake during each collection period, IL- $1 \alpha$ concentration was divided by the total protein (TP) concentration $(\mu \mathrm{g} / \mathrm{ml})$ measured in the samples to calculate the IL-1 $\alpha /$ TP-ratio. To measure the amount of total protein, the Thermo Scientific ${ }^{\text {TM }}$ Micro BCA Protein Assay Kit (Pierce Biotechnology) was used with a detection range of $0.5-20 \mu \mathrm{g} / \mathrm{ml}$. 


\section{Biophysical analysis}

Cutaneous blood flow measurements were performed with the Moor LDI2 burn imager. A single point laser beam scanned the skin back and forth creating a cutaneous blood cell flux map to measure the mean velocity of the red blood cells. The laser (infra-red, $785 \mathrm{~nm}$ wavelength) probed the full dermal thickness, approximately 1-2 $\mathrm{mm}$ into the skin. The acquisition depth of the laser is tissue dependent and influenced by the amount of pigmentation. A region of interest (ROI) of $30 \mathrm{~mm}$ x $30 \mathrm{~mm}$ was selected in the cutaneous blood cell flux map and the mean cutaneous blood cell flux of the ROI was calculated with the Moor software version 5.3.

\section{Statistical methods}

GraphPad Prism 5 (GraphPad Software Inc. San Diego, CA) and SPSS statistics version 20 (SPSS Inc, Chigaco,IL) for Windows were used to analyse the data.

Normality was tested with the D'Agostino Pearson Omnibus test. A parametric test was used when data was distributed normally, or a non-parametric test for a nonnormal distribution. The non-parametric Wilcoxon signed rank test was used to determine significance between paired comparisons.

To determine which dressing was most effective, the increase compared with baseline values was calculated for control (without dressing) and dressing values. These calculated values were used to determine the relative change (in \%) between the dressing and their subsequent control value to compensate for intra-individual variability. The non-parametric Friedmann test followed by Dunn's multiple comparison test was used to determine statistically significant differences in the increase (in \%) between the three dressings. Data are expressed as mean \pm standard deviation or median + interquartile range. For graphical purposes, the results are presented as means \pm standard error of mean (SEM). A statistical significance level of $5 \%$ was prescribed $(\mathrm{P}<0.05)$.

\section{Results}

Ten healthy male Caucasian volunteers were included in this study. Every volunteer finished the three test days with no drop-outs or missing data reported. Mean (SEM) age was $22.5 \pm 1.6$; mean height was $1.87 \pm 0.1$ metres; mean weight was $78.0 \pm 11.8 \mathrm{~kg}$ with a BMI of $22.3 \pm 2.4 \mathrm{~kg} / \mathrm{m}^{2}$.

\section{IL-1 $\alpha /$ TP-ratio}

The IL-1 $\alpha /$ TP-ratio after the application of pressure and shear was significantly lower when the dressings were attached to the skin compared with their own control 
measurement (Figure 6.4A, B and C). When the three dressings were compared to each other, the calculated change in IL-1 $\alpha$ /TP (in \%) was statistically lower when the Mepilex ${ }^{\circledR}$ border dressing was used compared to the Allevyn Adhesive dressing (Figure 6.7A, $\mathrm{P}<0.01$ ), but was not statistically different compared to the Aquacel ${ }^{\mathrm{TM}}$ foam dressing (Figure 6.2D, $P>0.05$ ). No statistically significant differences were found between the Allevyn Adhesive and the Aquacel ${ }^{\mathrm{TM}}$ foam dressing (Figure 6.7A, $\mathrm{P}>0.05$ ).

A

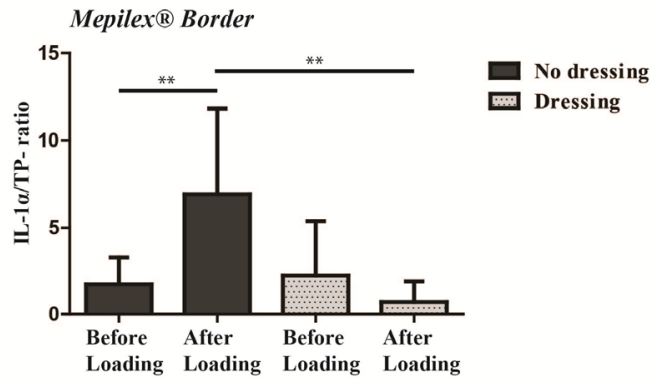

B

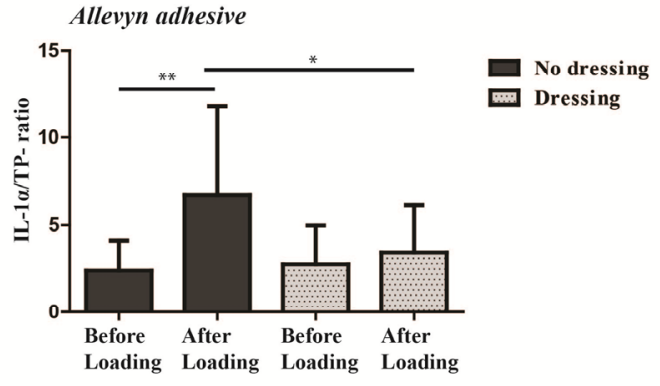

C

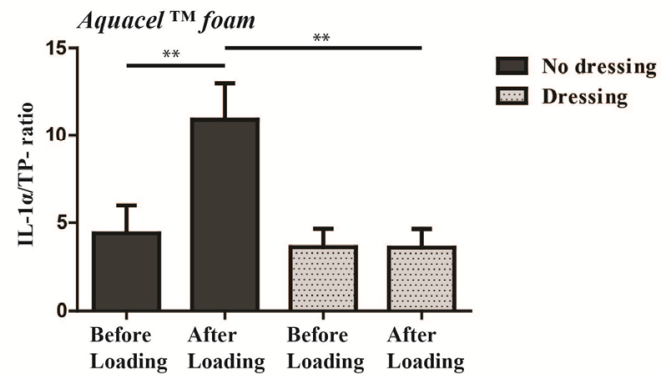

Figure 6.4 IL-1 $\alpha /$ TP-ratio $\left(\times 10^{-6)}\right.$. presented as mean (SEM). ** indicates $\mathrm{P}<0.001$ (Wilcoxon signed rank test). ${ }^{*}$ indicates $\mathrm{P}<0.05,{ }^{* *}$ indicates $\mathrm{P}<0.01$ (Wilcoxon signed rank test).

\section{Cutaneous blood cell flux}

Typical perfusion images are presented in Figure 6.5. When a combined loading of pressure and shear was applied to the skin, the cutaneous blood cell flux was significantly lower when the Mepilex ${ }^{\circledR}$ border dressing or the Aquacel ${ }^{\mathrm{TM}}$ foam dressing 
was attached to the skin compared to the control measurement without a dressing (Figure 6.6A and $\mathrm{B}, \mathrm{P}<0.001$ ). Although the Allevyn Adhesive dressing reduces the amount of post- load reactive hyperaemia too, the mean difference of $114.6 \pm 173.1$ arbitrary units (AU) was not significantly lower compared to its own control measurement (Figure 6.6C. $\mathrm{P}>0.05$ ). Relative change in cutaneous blood cell flux (in \%) was not statistically different between the Mepilex ${ }^{\circledR}$ border dressing and Aquacel ${ }^{\mathrm{TM}}$ foam dressing. However, both dressings induced significantly less post- load cutaneous blood cell flux than the Allevyn Adhesive dressing (Figure 6.7B. $P<0.01$ and $\mathrm{P}<0.001$, respectively).

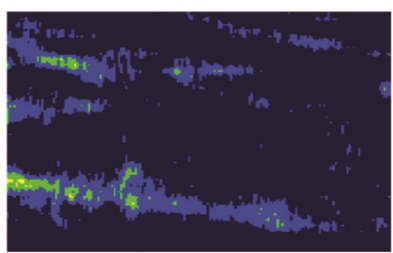

Baseline measurement

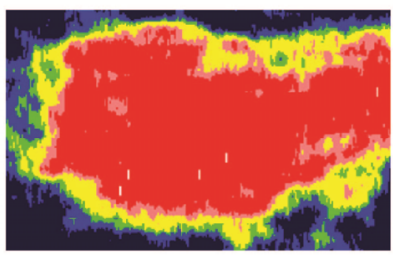

Post-load measurement without dressing

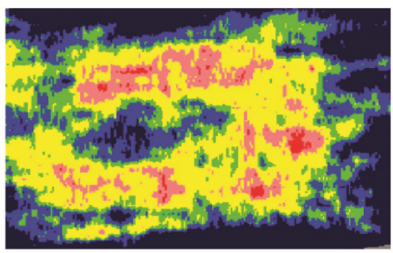

Post-load measurement with Mepilex® Border

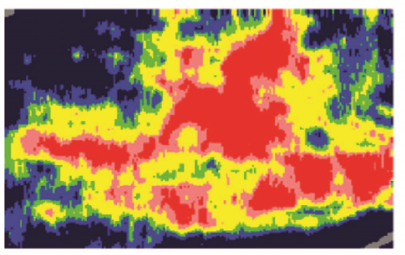

Post-load measurement with Allevyn Adhesive

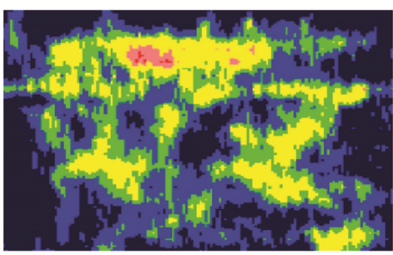

Post-load measurement with Aquacel ${ }^{\mathrm{TM}}$ Foam
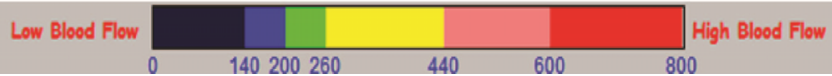

Figure 6.5 Cutaneous Blood cell flux images created with the Moor LDI2- burn imager 
A

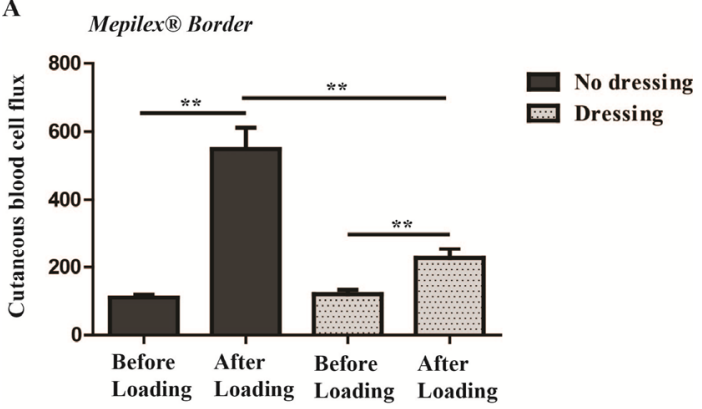

B

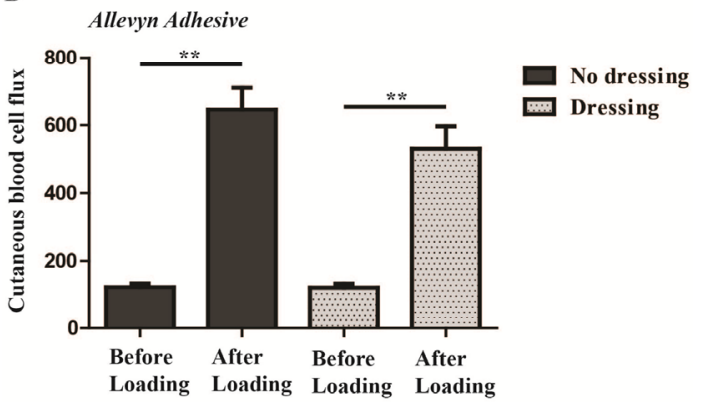

C

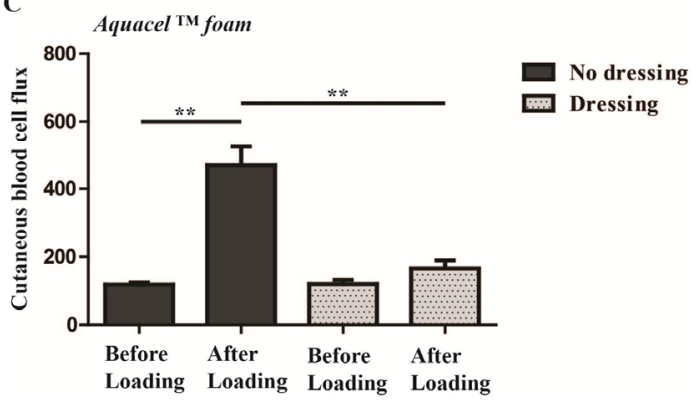

Figure 6.6 Cutaneous Blood Cell Flux (in arbitrary units) presented as mean (SEM). ${ }^{* *}$ indicates $\mathrm{P}<0.001$ (Wilcoxon signed rank test).

\section{Lactate}

No statistically significant increase in lactate concentration was measured when pressure and shear was applied to the skin for 30 minutes compared with baseline measurement $(28.8 \pm 18.0 \mu \mathrm{mol} / \mathrm{l}$ vs. $37.3 \pm 25.1 \mu \mathrm{mol} / \mathrm{l}, \mathrm{P}=0.07)$. Therefore, lactate could not be used as a measure to evaluate shear relieving capacities of dressings with this model. 
A

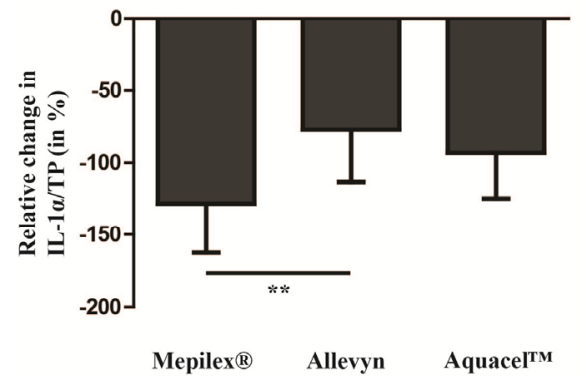

B

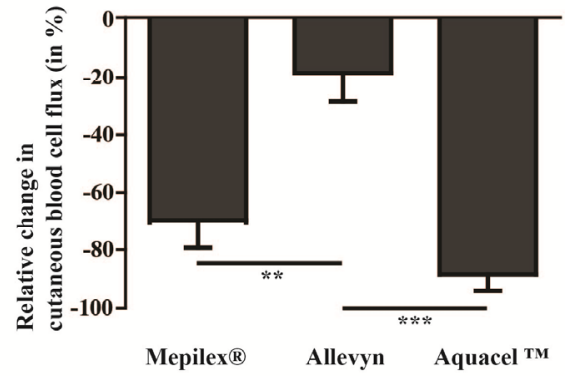

Figure 6.7 Effectiveness of the dressings compared to each other. The increase compared with baseline values was calculated for control (without dressing) and dressing values. These calculated values were used to determine the relative change (in \%) between the dressing and their subsequent control value to compensate for intra- individual variability. A. IL-1 $\alpha /$ TP increase in percentage. B. Cutaneous blood cell flux increase in percentage. ${ }^{* *}$ Indicates $\mathrm{P}<0.01, * * *$ indicates $\mathrm{P}<0.001$ (Friedmann test with Dunn's multiple comparison test).

\section{Discussion}

The factor shear has been known to play an important role in the development of pressure ulcers for a long tim ${ }^{6,8,9}$. With the help of a new validated shear mode ${ }^{18}$ we were able to evaluate the protective effect of three commercially available foam dressings against a combined loading of pressure and shear. The Mepilex ${ }^{\circledR}$ border, Allevyn Adhesive and Aquacel ${ }^{\mathrm{TM}}$ foam dressings were chosen, because the manufacturers claim that these dressings can relieve the amount of shear on the skin and can be used as an addition to pressure ulcer preventive strategies. Indeed, this study demonstrated that all three foam dressings reduced epidermal IL- $1 \alpha$ release and two foam dressing induced less post-load reactive hyperaemia in reaction to pressure and shear loading on the skin in healthy participants. Therefore, this study is the first to demonstrate that foam dressings can relieve the effects of pressure in combination with shear in vivo.

The increase in IL-1 $\alpha /$ TP- ratio was used as a measure of skin damage after loading on the $\operatorname{skin}^{20-23}$. IL-1 $\alpha$ is a damage-associated molecular pattern and stored in in the healthy keratinocytes, there it will be rapidly released in the extracellular matrix when the keratinocytes becomes mechanically damaged ${ }^{21,23,24}$. In the previous study ${ }^{18}$, the IL-1 $\alpha$ response was particularly pronounced after 30 minutes of pressure and shear loading. Once released in the extracellular matrix, it induces the production and secretion of other cytokines which are essential in the process of wound healing. Although IL-1 $\alpha$ is just a single measure of the inflammatory reaction to skin damage, it seems an excellent way to measure predominant damage in epithelial cells ${ }^{25}$. Furthermore, Wood and colleagues ${ }^{24}$ demonstrated that IL- $1 \alpha$ is immediately released 
from keratinocytes when the skin barrier is disrupted. Clearly, the results of the present study shows that foam dressings protects the skin against direct damage caused by pressure in combination with shear, because all three foam dressings were capable to reduce the post-load IL-1 $\alpha$ release.

Another important finding was the reduction of post- load reactive hyperaemia when the Mepilex ${ }^{\circledR}$ border dressing or Aquacel ${ }^{\mathrm{TM}}$ foam dressing were applied to protect the skin. In the present study, the reactive hyperaemic response was measured with a Laser Doppler Flowmeter and expressed in cutaneous blood cell flux (in arbitrary units). Post-load reactive hyperaemia is the increase in cutaneous blood flow and normally appears as an reaction on tissue hypoxia and tissue deformation ${ }^{26-28}$. It is interesting to note that the concentration of lactate, which was used as a measure for tissue hypoxia, was not significantly higher after a combined loading of pressure and shear for 30 minutes compared with baseline measurements. Therefore, it is likely that the reduction in reactive hyperaemia after the application of the Mepilex ${ }^{\circledR}$ border dressing and Aquace ${ }^{\mathrm{TM}}$ foam dressing was mainly caused by protection against tissue deformation rather than protection against tissue ischemia.

Normally, two pathophysiological pathways are responsible for cell damage caused by mechanical loading ${ }^{29}$. First, mechanical loading can activate a slow process by compressing blood and lymph vessels. Consequently, tissue becomes ischaemic, metabolic waste products (for example lactate) will accumulate and the interstitial $\mathrm{pH}$ will decrease. However, the first signs of histological cellular damage will only be visible after a couple of hours ${ }^{30,31}$.

Second, a much faster process may occur when mechanical loading on the skin becomes high. In this case, the cellular membrane and cytoskeleton becomes disrupted and the first signs of cellular damage are already visible within 10 minutes ${ }^{32}$. This could explain why the concentration of lactate was not significantly increased within $\mathbf{3 0}$ minutes, as it is associated with slow ischaemic damage. In contrast, the IL$1 \alpha$ response is more likely to be associated with the faster direct deformation damage.

In the present study, the Mepilex ${ }^{\circledast}$ border dressing and Aquacel ${ }^{\mathrm{TM}}$ foam dressing performed better than the Allevyn Adhesive dressing. A possible explanation could be the fact that the the Mepilex ${ }^{\circledR}$ border dressing and Aquacel ${ }^{\mathrm{TM}}$ foam dressing are both multi- layered foam dressings compared with the single- layered Allevyn Adhesive dressing. This is in line with an earlier observation based on a theoretical finite element model of Levy and colleagues ${ }^{33}$. They reported a higher decrease in tissue strains when a multi- layered dressing was used compared with a single layered dressing, because the internal deformation in the dressing becomes higher when a multi-layered dressing is used. However, because of the heterogeneous nature (different material combinations, geometry not fully comparable) of the dressings used in the present study, it is difficult to determine the Mepilex ${ }^{\circledR}$ border and Aquacel ${ }^{\mathrm{TM}}$ foam dressing were more effective in improving the effect of shear on skin viability due to their multi-layered foam design. Many other aspects other aspects like 
material stiffness, material thickness, chemistry of skin contact layer may have influence on the shear absorbing capacities of dressings as well.

Recently, some clinical evidence shows that wound dressings can reduce the incidence of pressure ulcers when used as an adjunct to pressure ulcer prevention strategies ${ }^{34-36}$. Unfortunately, these studies had a high risk of bias and firm conclusions should not be drawn from these trials ${ }^{37,38}$.

Several ex vivo studies evaluated the shear reducing capacities of different types of dressings $^{15-17}$. Ohura and colleagues ${ }^{17}$ were the first to investigate the shear relieving capacities of wound dressings. In their study, shear was applied over an excised piece of porcine skin with a sensor placed underneath it. A lower amount of shear force was measured when different types of wound dressing were applied over the porcine skin. A more recent study performed by Call and colleagues ${ }^{15}$ demonstrated in a laboratory bench setting the potential shear reducing capacities of different types foam dressings as well. Although, both studies demonstrated that dressings are capable in reducing the amount of shear, they did not investigate if foam dressings could improve the effect of shear on skin viability.

Derler and colleagues ${ }^{39}$ demonstrated that the friction coefficient between the skin interface and textile can be reduced with a novel medical bed sheet in comparison with a normal hospital bed sheet especially under wet conditions. Skin moisture represents an important extrinsic factor in the development of pressure ulcers. When skin becomes wet, the friction coefficient increases, which in turn, could lead to larger shear strains on the skin surface and deeper skin layers. Moreover, moisture uptake by the skin induces skin softening and smoothening which makes the skin particularly for skin barrier damage ${ }^{40}$, so foam dressings should be able to handle moisture (in the form of sweat) before they are used as a preventive measure. Although the fluidhandling capacity of the foam dressings was not investigated in the present study, several other studies demonstrated that foam dressings have good fluid handling properties $^{41-43}$.

In the previous study ${ }^{18}$, the pressure of $2.5 \mathrm{kPa}$ and shear of $14.5 \mathrm{~N}$ produced significant changes on the robust parameters derived from the measurement techniques after only 30 minutes of loading. In addition, it is a realistic representation of the clinical setting where patients are exposed to a mean physiological shear between 5-20 $\mathrm{N}$ and a mean pressure between 2.5-8.0 kPa on a standard support mattress ${ }^{13}$. However, the results of the present study cannot be directly translated to patients at risk for pressure ulcers, who are normally critically ill, have multiple comorbidities, high age, and/or other underlying diseases. Another limitation is the short loading period of 30 minutes, because loading periods could be much longer (up to several hours) in a clinical setting. However, such a long period of loading was undesirable, to avoid severe skin damage in the human volunteers. The relatively short loading period of only 30 minutes was possibly the main reason that lactate could not be used as a measure for pressure and shear in this study. In addition, the 
type of loading included only continuous loading whereas loading in a clinical setting loading includes alternating short-term loading too.

Another comment needs to be placed on the fixation strength of the Aquacel ${ }^{\mathrm{TM}}$ foam dressing. The dressings did not remain in place when they were loaded with the prescribed amount of pressure and shear, because only the borders adhere to the skin to reduce pain and trauma upon removal. In a clinical setting, the use of this dressing could lead to problems if not fixated properly.

Recommendations for optimisation of dressings design should include a multi-layered composition a low friction coefficient of the outer layer and good fluid handling capacities. However, to investigate if multi-layered dressings are indeed better in absorbing shear in comparison with single-layered dressings, future research should include dressings made out of exactly the same materials (i.e. foam, skin contact layer and backings) with only different numbers of foam layers. Unfortunately, these dressing are currently not commercially available.

In conclusion, this is the first in vivo study to demonstrate that foam dressings can improve the effects of shear on skin viability in humans. Therefore, they might be useful in pressure ulcer preventive strategies. Secondly, the multi- layered foam dressings performed better in improving the effects of shear on skin viability when compared to a single-layered foam dressing in this study. 


\section{References}

1. Petzold T, Eberlein-Gonska M, Schmitt J. Which factors predict incident pressure ulcers in hospitalized patients? A prospective cohort study. Br L Dermatol. 2014;170(6):1285-1290.

2. Schuurman JP, Schoonhoven L, Defloor T, van Engelshoven I, van Ramshorst B, Buskens E. Economic evaluation of pressure ulcer care: a cost minimization analysis of preventive strategies. Nurs Econ. 2009;27(6):390-400, 415.

3. Filius A, Damen TH, Schuijer-Maaskant KP, Polinder S, Hovius SE, Walbeehm ET. Cost analysis of surgically treated pressure sores stage III and IV. J Plastic Reconstr Aesthet Surg. 2013;66(11): 1580-1586.

4. Lyder CH, Grady J, Mathur D, Petrillo MK, Meehan TP. Preventing pressure ulcers in Connecticut hospitals by using the plan-do-study-act model of quality improvement. Jt Comm J Qual Saf. 2004;30(4):205-214.

5. Gillespie BM, Chaboyer WP, McInnes E, Kent B, Whitty JA, Thalib L. Repositioning for pressure ulcer prevention in adults. The Cochrane database of systematic reviews. 2014;4:CD009958.

6. Bennett L, Kavner D, Lee BK, Trainor FA. Shear vs pressure as causative factors in skin blood flow occlusion. Arch Phys Med Rehabil. 1979;60(7):309-314.

7. Goossens RH, Zegers R, Hoek van Dijke GA, Snijders CJ. Influence of shear on skin oxygen tension. Clin Physiol. 1994;14(1):111-118.

8. Reichel SM. Shearing force as a factor in decubitus ulcers in paraplegics. J Am Med Assoc. 1958;166(7):762-763.

9. Dinsdale SM. Decubitus ulcers: role of pressure and friction in causation. Arch Phys Med Rehabil. 1974;55(4):147-152.

10. Linder-Ganz E, Gefen A. The effects of pressure and shear on capillary closure in the microstructure of skeletal muscles. Ann Biomed Eng. 2007;35(12):2095-2107.

11. Mclnnes E, Jammali-Blasi A, Bell-Syer SE, Dumville JC, Middleton V, Cullum N. Support surfaces for pressure ulcer prevention. The Cochrane database of systematic reviews. 2015;9:CD001735.

12. Gefen A, Farid KJ, Shaywitz I. A review of deep tissue injury development, detection, and prevention: shear savvy. Ostomy Wound Manage. 2013;59(2):26-35.

13. Mimura M, Ohura T, Takahashi M, Kajiwara R, Ohura N, Jr. Mechanism leading to the development of pressure ulcers based on shear force and pressures during a bed operation: influence of body types, body positions, and knee positions. Wound Repair Rgen. 2009;17(6):789-796.

14. Black J, Clark M, Dealey C, et al. Dressings as an adjunct to pressure ulcer prevention: consensus panel recommendations. Int Wound J. 2015;12(4):484-488.

15. Call E, Pedersen J, Bill B, et al. Enhancing pressure ulcer prevention using wound dressings: what are the modes of action? Int Wound J. 2015;12(4):408-413.

16. Ohura N, Ichioka S, Nakatsuka T, Shibata M. Evaluating dressing materials for the prevention of shear force in the treatment of pressure ulcers. J Wound Care. 2005;14(9):401-404.

17. Ohura T, Takahashi M, Ohura N, Jr. Influence of external forces (pressure and shear force) on superficial layer and subcutis of porcine skin and effects of dressing materials: are dressing materials beneficial for reducing pressure and shear force in tissues? Wound Repair Regen. 2008;16(1):102-107.

18. de Wert LA, Bader DL, Oomens CW, Schoonhoven L, Poeze M, Bouvy ND. A new method to evaluate the effects of shear on the skin. Wound Repair Regen. 2015;23(6):885-890.

19. Perkins MA, Osterhues MA, Farage MA, Robinson MK. A noninvasive method to assess skin irritation and compromised skin conditions using simple tape adsorption of molecular markers of inflammation. Skin Res Technol. 2001;7(4):227-237.

20. Bronneberg D, Spiekstra SW, Cornelissen LH, et al. Cytokine and chemokine release upon prolonged mechanical loading of the epidermis. Exp Dermatol. 2007;16(7):567-573.

21. Cornelissen LH, Bronneberg D, Bader DL, Baaijens FP, Oomens CW. The transport profile of cytokines in epidermal equivalents subjected to mechanical loading. Ann Biomed Eng. 2009;37(5):1007-1018.

22. D B. In vivo detection of grade I pressure ulcers.

23. Lee RT, Briggs WH, Cheng GC, Rossiter HB, Libby $P$, Kupper T. Mechanical deformation promotes secretion of IL-1 alpha and IL-1 receptor antagonist. J Immunol. 1997;159(10):5084-5088. 
24. Wood LC, Elias PM, Calhoun C, Tsai JC, Grunfeld C, Feingold KR. Barrier disruption stimulates interleukin-1 alpha expression and release from a pre-formed pool in murine epidermis. J Invest Dermatol. 1996;106(3):397-403.

25. Suwara MI, Green NJ, Borthwick LA, et al. IL-1alpha released from damaged epithelial cells is sufficient and essential to trigger inflammatory responses in human lung fibroblasts. Mucosal Immunol. 2014;7(3):684-693.

26. Bliss MR. Hyperaemia. J Tissue Viability. 1998;8(4):4-13.

27. Iredahl F, Lofberg A, Sjoberg F, Farnebo S, Tesselaar E. Non-Invasive Measurement of Skin Microvascular Response during Pharmacological and Physiological Provocations. PloS One. 2015;10(8):e0133760.

28. Liao F, Burns S, Jan YK. Skin blood flow dynamics and its role in pressure ulcers. J Tissue Viability. 2013;22(2):25-36.

29. Stekelenburg A, Gawlitta D, Bader DL, Oomens CW. Deep tissue injury: how deep is our understanding? Arch Phys Med Rehabil. 2008;89(7):1410-1413.

30. Gawlitta D, Li W, Oomens CW, Baaijens FP, Bader DL, Bouten CV. The relative contributions of compression and hypoxia to development of muscle tissue damage: an in vitro study. Ann Biomed Eng. 2007;35(2):273-284.

31. Stekelenburg A, Strijkers GJ, Parusel H, Bader DL, Nicolay K, Oomens CW. Role of ischemia and deformation in the onset of compression-induced deep tissue injury: MRI-based studies in a rat model. J Appl Phys. 2007;102(5):2002-2011.

32. Oomens CW, Bader DL, Loerakker S, Baaijens F. Pressure induced deep tissue injury explained. Ann Biomed Eng. 2015;43(2):297-305.

33. Levy A, Frank MB, Gefen A. The biomechanical efficacy of dressings in preventing heel ulcers. J Tissue Viability. 2015;24(1):1-11.

34. Santamaria N, Gerdtz M, Sage S, et al. A randomised controlled trial of the effectiveness of soft silicone multi-layered foam dressings in the prevention of sacral and heel pressure ulcers in trauma and critically ill patients: the border trial. Int Wound J. 2015;12(3):302-308.

35. Brindle CT, Wegelin JA. Prophylactic dressing application to reduce pressure ulcer formation in cardiac surgery patients. J Wound Ostomy Continence Nurs. 2012;39(2):133-142.

36. Chaiken N. Reduction of sacral pressure ulcers in the intensive care unit using a silicone border foam dressing. J Wound Ostomy Continence Nurs. 2012;39(2):143-145.

37. Moore ZE, Webster J. Dressings and topical agents for preventing pressure ulcers. The Cochrane database of systematic reviews. 2013;8:CD009362.

38. Clark M, Black J, Alves $\mathrm{P}$, et al. Systematic review of the use of prophylactic dressings in the prevention of pressure ulcers. Int Wound J. 2014;11(5):460-471.

39. Derler S, Rotaru GM, Ke W, et al. Microscopic contact area and friction between medical textiles and skin. J Mech Behav Biomed Mater. 2014;38:114-125.

40. Gerhardt LC, Strassle V, Lenz A, Spencer ND, Derler S. Influence of epidermal hydration on the friction of human skin against textiles. J R Soc Interface. 2008;5(28):1317-1328.

41. Thomas S, Young S. Exudate-handling mechanisms of two foam-film dressings. J Wound Care. 2008;17(7):309-315.

42. Young $S B A$, Milne J. Use of ultrasound to characterise the fluid-handling characteristics of four foam dressings. J Wound Care. 2007;425(8):430-431.

43. Thomas S. Laboratory findings on the exudate-handling capabilities of cavity foam and foam-film dressings. J Wound Care. 2010;19(5):192, 194-199. 



\section{Chapter}

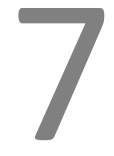

The prevention of sacral pressure ulcers in high-risk patients with the use of a sacral wound dressing: a randomized clinical trial

L.A. de Wert, B.A.B. Essers, A.A.G. Kessels, R.R. Vogels, M. Poeze, L. Schoonhoven, N.D. Bouvy 


\section{Abstract}

\section{Objectives}

Prophylactic wound dressings could be an easy addition in of pressure ulcers preventive strategies. Therefore, we investigated the effect of sacral dressing as an addition to current preventive strategies to prevent sacral pressure ulcers.

\section{Methods}

In this single-centre, randomized trial, a sample of 870 patients as the targeted number of inclusion was calculated. Inclusion criteria were: a Prepurse score $\geq 20$ or a Braden score $<19$ and immobility for $\geq 3$ days. The dressing group received usual care plus a sacral dressing and the control group received usual care only. Follow-up was performed until six weeks, hospital discharge or death. The Primary outcome was: Sacral pressure ulcer category 1-4. Secondary outcomes were: Daily visual analog scale-scores for pain and discomfort, adverse device effects and cost-effectiveness.

\section{Results}

This trial was prematurely terminated after we included 117 Patients in the dressing group and 127 in the control group, because of the low incidence of clinically relevant pressure ulcers. The total incidence of sacral pressure in the dressing group and control group was; $3.4 \%$ vs. $5.5 \%$, $\mathrm{P}=0.5$. The incidence of category $2-3$ pressure ulcers in the dressing and control group was $2.5 \%$ vs $0.8 \%, P=0.4$. Median VAS scores (day $3-8$ ) were low in the dressing and control group: 0.05 (IQR 0-4.8) vs. 0.03 (IQR 0-7,9), P= 0.8 for sacral pain and 0.05 (IQR 0-4.1) vs. 0,07 (IQR 0-9.1), $\mathrm{P}=0.8$ for sacral discomfort.

The incremental cost to prevent a sacral pressure ulcer with the dressing strategy is $€ 2238$, the probability that the dressing is cost-effective for this amount is $50 \%$.

This study showed a high number of device deficiencies, because the dressing curled or was totally released in 62 patients.

\section{Conclusion}

We do not have enough power to judge if there could be a prophylactic effect of the dressing, Because of the unexpected low incidence of sacral pressure ulcers. The trial was prematurely terminated, because the dressing could not be of clinical importance in in high-risk hospitalized patients. Therefore, the dressing was not a necessary addition to the current preventive pressure ulcer strategies in this setting. 


\section{Introduction}

A pressure ulcer is a localized tissue injury to the superficial layer of the skin and/ or the underlying tissue over a bony prominence and is most often seen in the elderly, disabled and critically ill patients. ${ }^{1-3}$ Pressure ulcers are most likely to develop in skin areas exposed to pressure and shear and usually appear at the sacral area and heels. Other places at risk of the development of pressure ulcers are: the head, ankles, hips and elbows. ${ }^{4,5}$ The European Pressure Ulcer Advisory Panel (EPUAP) guidelines state that pressure ulcers can be divided into four categories (category 1 to 4). ${ }^{3}$

In spite of the fact that the basic mechanisms of preventing pressure ulcers seem quite simple, pressure ulcers remain a major healthcare problem. With prevalence rates reported up at $7-38 \%$ of patients in hospitals ${ }^{2,6,7}$, it is a commonly seen injury. Since the occurrence of a pressure ulcer results in more pain, lower quality of life and a longer hospital stay ${ }^{8,9}$, patients and caregivers would rather avoid these injuries.

To reduce pressure in patients, regular turning and repositioning schedules are recommended and various devices including foam mattresses, air-suspension beds and air fluidized beds have been developed. While these measures have proven to be effective $^{10,11}$, the costs of these measures can be up to several hundred euros a day and turning a patient every four hours requires considerable effort from the professionals $^{12,13}$.

Recently, there has been interest in the prophylactic use of wound dressings in the prevention of pressure ulcers ${ }^{14-18}$. Different observational studies and randomized trials claim these wound dressings may be effective in the prevention of pressure ulcers. A recent Cochrane review, however, concludes that the results of these randomized trials are compromised by the low - quality of these trials and did not report patient related experiences, adverse device effects and economic evaluations ${ }^{19}$.

We, therefore, conducted a single-center, prospective, randomized trial, to investigate the prevention of sacral pressure ulcers using a soft silicone sacral dressing (Mepilex ${ }^{\circledR}$ Border Sacrum) in high-risk patients. Preventive measures, adverse device effects, device deficiencies and daily visual analog scale (VAS)-scores for pain and discomfort were also recorded.

\section{Methods}

\section{Study design}

This study was a single-centre, randomized controlled trial, originally powered at 870 patients with a high risk of developing sacral pressure ulcers, admitted to the Maastricht University Medical Centre (MUMC+). 
Patients were recruited between September $1^{\text {st }} 2012$ and September $1^{\text {st }}$ 2013. The research protocol was approved by the medical ethics committee of the MUMC+ and was registered in the database of clinicaltrials.gov NCT01640418. The study was conducted in compliance with the ethical rules for human experimentation that are stated in the Declaration of Helsinki, and was reported conform the CONSORT statement. $^{20}$

All newly admitted adult patients who had a Prepurse score $\geq 21^{21}$ or a Braden score $<19$ and were expected to be immobile (bed or chair bound) for 3 days or more, were informed about this study by a research nurse and a patient information folder. Immobile was defined as the inability to walk at least two times a day outside the room or inside the room at least every two hours. Patients who were mobile at hospital admission but were expected to be immobile for 3 days or more because of planned surgery could also participate in this study. Exclusion criteria were age $<18$ years, pre-existing sacral pressure ulcer, pre-existing trauma to the sacrum, inability to speak Dutch, mentally disabled patients or patients who went directly to the ICU after hospital admission.

Patients were given the opportunity to decide to participate in this study but informed consent and participation at this study had to take place within 48 hours after hospital admission or before going to the operating room (for surgical patients). Randomization of patients was performed online via TENALEA in a 1:1 ratio by a member of the research team directly after written informed consent was obtained. Patients were randomly assigned to the dressing group or control group. Both patients and members of the research team were not blinded.

\section{Intervention}

Patients in the dressing group received a five-layered soft silicone self-adherent sacral dressing (Mepilex ${ }^{\circledast}$ Border Sacrum dressing, Mölnlycke Healthcare AB, Göteborg, Sweden). Immobile patients received this dressing directly after randomization by a research nurse. Surgical patients who were mobile at hospital admission received this dressing just before they went to the OR. Two dressing sizes were available $(18 \times 18 \mathrm{~cm}$ and $23 \times 23 \mathrm{~cm}$ ) and the size was matched to the patient's body size. The dressing was replaced at least every four days or more frequently when necessary in cases when the dressing was soiled, displaced, curled or not in the right place and this was recorded by a member of the research team. The dressing was removed when a patient became mobile again. All patients in the control and intervention groups were treated according to the standard hospital protocol for the prevention of sacral pressure ulcers of the MUMC+. This includes frequent repositioning (six times a day) of immobile patients and the use of specialized mattresses to prevent pressure ulcers. The study protocol did not intervene with any procedures already planned during hospital admission. In case of the development of a pressure ulcer, the patient was 
treated according to standard hospital care, regardless of the group the patient was assigned to.

\section{Outcomes and data collection}

The primary outcome was the development of a sacral pressure ulcer category 1-4. Secondary outcomes were: a visual analog score for both pain and discomfort in general and at the sacral area, Euroqol VAS-scores, total number of dressing changes and preventive measures.

At hospital admission information about age, sex, body-mass index (BMI), American Society of Anesthesiologists (ASA)-score, expected surgery, medical diagnosis, nutritional status, co morbidities, activity, mobility, incontinence and smoking behavior and was registered.

Data collection was performed by a research nurse and recorded in an online data management system. During the clinical visits, the research nurse inspected the sacral area of the patients for the presence of pressure ulcers (every two days). When a pressure ulcer appeared, the pressure ulcer was graded according to the EPUAP guidelines ${ }^{3}$. An independent nurse practitioner, specialized in pressure ulcer wound care, was called in to check the decision of the research team. Every day the research team assessed if a patient became mobile again. Daily VAS-scores for total pain and discomfort and sacral pain and discomfort with 0 for no pain or discomfort and 10 the worst imaginable pain or discomfort were rated and recorded by the patients. Information about preventive measures was collected by asking the general nurses responsible for the patients daily care. At two points (baseline and discharge) the patients were asked to rate their health according to the EuroQol quality of life VAS scale with 0 the worst imaginable health status and 100 the best imaginable health status. The clinical visits were performed until 6 weeks of hospital admission, hospital discharge, voluntary withdrawal of patients or death.

\section{Cost calculation methods}

We registered resource use and subsequently calculated the total costs per patient during their hospital admission. These costs consisted of: repositioning costs, specialized mattress costs and dressing costs. In our hospital an immobile patient was repositioned six times a day, the mean repositioning time of 7.9 (95\% $\mathrm{Cl} 7.3-8.6$ ) minutes was based on a study by Schuurman et al. ${ }^{12}$, so this gives a total repositioning time of 47.4 minutes a day. The nursing costs per minute were $€ 0.34$ (Table 7.1) and the total repositioning costs per day are $€ 16.11$. We multiplied $€ 16.11$ with the number of days of immobility to calculate the total repositioning costs per patient. Two types of specialized matrasses were used during this trial; a alternating pressure mattress (Nimbus, Arjohuntleigh) and an open-pressurized mattress (Atmos Air, Company $\mathrm{KCl}$ ). Mattress costs per day were $€ 1.86$ for the Nimbus mattress and $€ 1.20$ for the Atmos mattress (Table 7.1). These numbers were multiplied with the total of 
days a patient needed one of these mattresses. We calculated the daily costs of these matresses according to the annuity method of depreciation ${ }^{22}$ with a $4 \%$ interest rate added to the cost price with a twelve year depreciation period for the Nimbus mattress and five year depreciation period for the Atmos mattress. To calculate the total dressing costs, we multiplied the cost of one dressing (€3.86, Table 7.1) with the number of dressings used per patient. The cost-effectiveness ratio was calculated by dividing the cost difference by the effect rate (difference in pressure ulcers). We calculated the weighted mean costs to treat one sacral pressure ulcer (category 1-3) in this trial based on the treatment costs of a pressure ulcer as calculated by Schuurman et al. ${ }^{12}$

Table 7.1 Costs, unit and sources

\begin{tabular}{lccl}
\hline Resource Use & Unit & Cost per unit, $€$ & Source \\
\hline $\begin{array}{l}\text { Nursing time } \\
\text { Repositioning }\end{array}$ & Minute & 0.34 & MUMC $^{\text {a }}$, Schuurman et. al. \\
$\begin{array}{l}\text { Mattress } \\
\text { Atmos }\end{array}$ & Day & 1.20 & MUMC $^{\text {a }}$, Dutch cost guidance \\
Nimbus & Day & 1.86 & MUMC* $^{22}$ Dutch cost guidance \\
Dressing & Piece & 3.86 & Mölnlycke Healthcare $^{22}$ \\
\hline
\end{tabular}

${ }^{a}$ Maastricht University Medical Centre, the Netherlands

\section{Statistical analysis}

This study was powered at 870 patients to find a $10 \%$ difference between groups. The software SPSS statistics version 20 was used to calculate means, standard deviation and frequencies of patient characteristics of all randomized patients. We used the two-sided Fisher exact test to compare the differences between the incidence of sacral pressure ulcers in the dressing and control group. We calculated the median VAS-scores (0-10) and the interquartile range (IQR) for total pain, sacral pain, total discomfort and sacral discomfort and calculated the median Euroqol VAS-scores and IQR at baseline and discharge. We used the Mann-Whitney $U$ test to compare differences in median VAS-scores. Since cost-data are generally skewed, a nonparametric bootstrap analysis for both cost and cost-effectiveness analysis (1000 replications) was performed ${ }^{23}$. The results of the bootstrap analysis for the ratio (i.e. the incremental costs to prevent an additional sacral pressure ulcer) is presented in a cost-effectiveness plane and an acceptability curve. The cost-effectiveness plane is a graphical presentation of four quadrants in which the additional costs and additional health outcome (in this case the prevention of a pressure ulcer) was compared with usual care. The acceptability curve shows the probability that the dressing strategy was cost-effective compared with usual care for different threshold values (i.e. the amount a hospital/ policymaker would be willing to pay to prevent one additional pressure ulcer). 


\section{Results}

\section{Study patients}

From September 2012 to September 2013, a total of 9918 patients were screened. The principal investigator decided to prematurely terminate the study after randomization of 253 patients, because of the unexpected low incidence of sacral pressure ulcers. A total of 253 patients underwent randomization, 122 patients were randomly assigned to the dressing group and 131 patients to the control group. Participant flowchart is summarized in Figure 7.1, including reasons for exclusion. Six patients in the dressing group did not receive a dressing at all, 24 patients discontinued the intervention because the dressing curled/ remained not in the right place, and seven patients discontinued the intervention after they occurrence of an ADE due to the wound dressing. Therefore, the research team decided to stop the intervention with the wound dressing (see: Device deficiencies and adverse device effects ( $A D E-s$ ) section). These patients were analyzed in the dressing group according to the intention- to treat principle. A total of 244 patients were included for statistical analysis; in this analysis we included seven patients who died during the trial and 31 drop outs. The baseline characteristics of all randomized patients are presented in Table 7.2.

\section{Pressure ulcer incidence}

A total of 11 patients developed a sacral pressure ulcer during their hospital admission. Four in the dressing group $(3,4 \%)$ and seven in the control group $(5,5 \%)$ $\mathrm{P}=0.5$ (two-sided Fisher's exact test).

Seven patients developed an category 1 sacral pressure ulcer; one in the dressing group and six in the control group. Three patients developed a category 2 sacral pressure ulcer; two in the dressing group and one in the control group. One patient in the dressing group developed a category 3 sacral pressure ulcer.

\section{Visual Analog Scores}

No significant difference in the median Euroqol VAS-scores (0-100) were found between the dressing and control group: 70.0 (IQR 0-100) vs. 70.0 (IQR 0-98), P=0.8 at baseline and 70.0 (IQR 0-98) vs. 68.5 (IQR 0-100), P=0.5 at discharge. The median VASscore for total pain (day 3-8) was 2.1 (IQR 0-9.6) in the dressing group and 1.8 (IQR 07.7) in the control group, $P=0.2$. The median VAS score for sacral pain (day 3-8) was 0.05 (IQR 0-4.8) in the dressing group and 0.03 (IQR 0-7.9) in the control group, $\mathrm{P}=0.8$. The median VAS score for total discomfort was (day 3-8) was 2.2 (IQR 0-9.5) in the dressing group and 2.4 (IQR 0-9.1) in the control group, $\mathrm{P}=0.9$ and for sacral discomfort (day 3-8) the median VAS score was 0.05 (IQR 0-4.1) in the dressing group and 0.07 (IQR 0-9.1) in the control group, $\mathrm{P}=0.8$. 


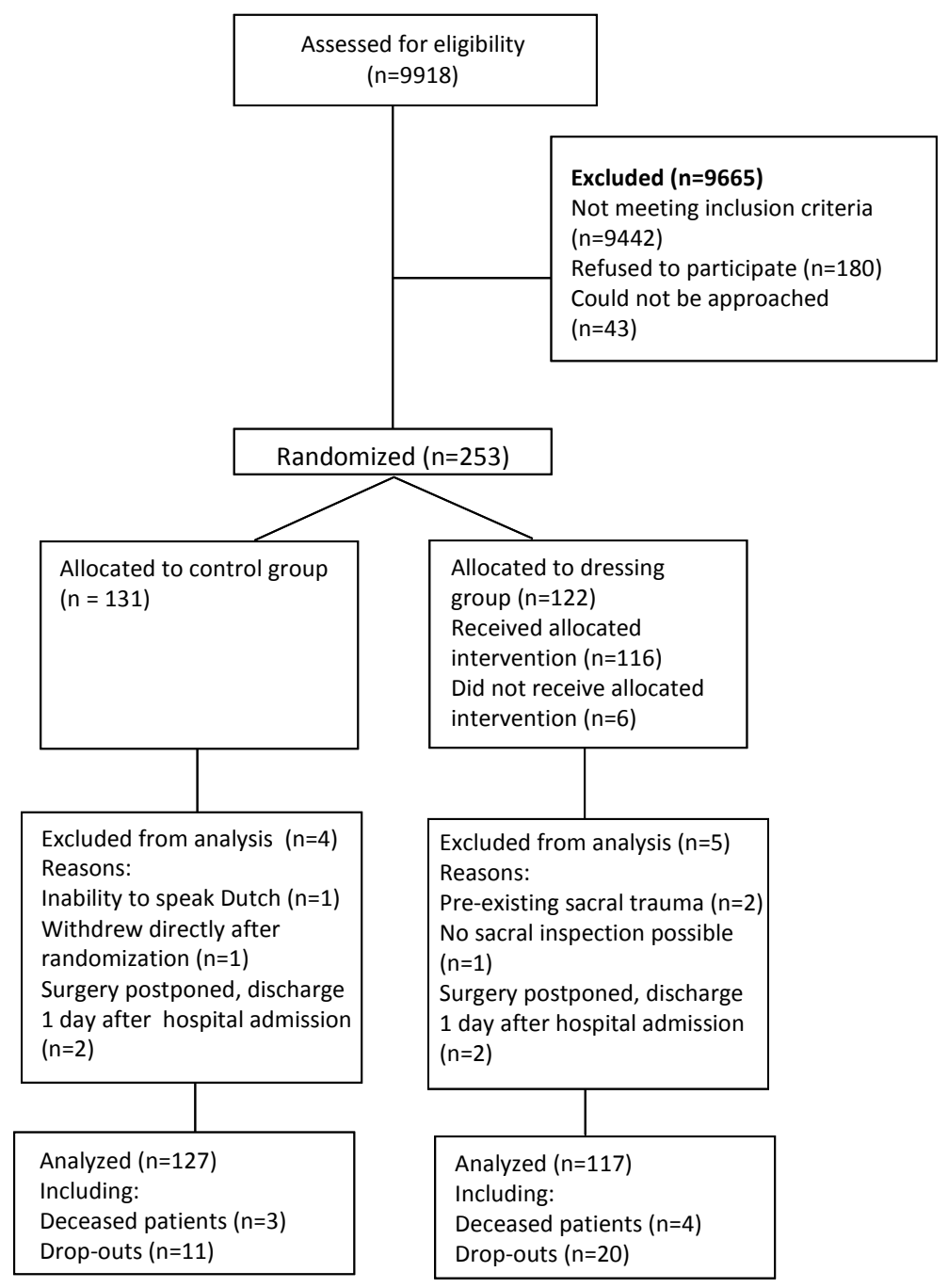

Figure 7.1 Participant flowchart 
Table 7.2 Baseline characteristics of all randomized patients

\begin{tabular}{|c|c|c|}
\hline & $\begin{array}{c}\text { Dressing } \\
n=122\end{array}$ & $\begin{array}{c}\text { Control } \\
n=131\end{array}$ \\
\hline Age- years mean $\left(s^{a}{ }^{a}\right)$ & $71.9 \pm 10.9$ & $70.9 \pm 9.8$ \\
\hline Male- no(\%) & $66(54.1)$ & $83(63.4)$ \\
\hline Prepurse- score mean (sd) & $23.6 \pm 3.8$ & $23.9 \pm 4.4$ \\
\hline Braden - score mean $(\mathrm{sd})^{\mathrm{b}}$ & $20.8 \pm 3.0$ & $21.1 \pm 2.9$ \\
\hline $\mathrm{BMI}-$ mean (sd) & $26.7 \pm 4.4$ & $26.4 \pm 4.9$ \\
\hline \multicolumn{3}{|l|}{ Medical diagnosis } \\
\hline Repiratory- no (\%) & $9(7.4)$ & $12(9.2)$ \\
\hline Cardiovascular- no (\%) & $55(45.1)$ & $53(40.5)$ \\
\hline Gastrointestinal- no (\%) & $29(23.8)$ & $28(21.4)$ \\
\hline Hepatic- no (\%) & $10(8.2)$ & $16(12.2)$ \\
\hline Nefrologicall- no (\%) & $2(1.6)$ & $2(1.5)$ \\
\hline Orthopedic/ trauma- no (\%) & $12(9.8)$ & $15(11.5)$ \\
\hline Gynecological- no (\%) & $1(0.8)$ & $2(1.5)$ \\
\hline Systematic disease- no (\%) & $1(0.8)$ & $3(2.3)$ \\
\hline Other- no (\%) & $3(2.5)$ & - \\
\hline Surgery- no (\%) & $111(91.0)$ & $114(87.0)$ \\
\hline \multicolumn{3}{|l|}{ ASA-score } \\
\hline I- no (\%) & $10(8.7)$ & $12(9.4)$ \\
\hline II- no (\%) & $40(34.8)$ & $39(30.5)$ \\
\hline III- no (\%) & $62(53.9)$ & $73(57.0)$ \\
\hline IV-no (\%) & $3(2.6)$ & $4(3.1)$ \\
\hline \multicolumn{3}{|l|}{ Nutritional status } \\
\hline Poor- no (\%) & $7(5.9)$ & $7(5.3)$ \\
\hline Inadequate- no (\%) & $9(7.6)$ & $9(6.9)$ \\
\hline Normal- no (\%) & $40(33.6)$ & $42(32.1)$ \\
\hline Perfect- no (\%) & $63(52.9)$ & $73(55.7)$ \\
\hline \multicolumn{3}{|l|}{ Comorbidities } \\
\hline Diabetes mellitus- no (\%) & $26(21.3)$ & $27(20.6)$ \\
\hline Hypertension- no (\%) & $55(45.1)$ & $57(43.5)$ \\
\hline Heart failure- no (\%) & $53(43.4)$ & $42(32.1)$ \\
\hline CVA- no (\%) & $8(6.6)$ & $9(6.9)$ \\
\hline COPD- no (\%) & $11(9.0)$ & $16(12.2)$ \\
\hline Aneurysm- no (\%) & $11(9.0)$ & $6(4.6)$ \\
\hline Urine continence- no (\%) & $113(92.6)$ & $120(91.6)$ \\
\hline Faeces continence- no (\%) & $114(93.4)$ & $115(87.8)$ \\
\hline \multicolumn{3}{|l|}{ Smoking behaviour } \\
\hline (ex) Smoker- no (\%) & $82(67.2)$ & $84(64.1)$ \\
\hline
\end{tabular}

${ }^{a} \mathrm{SD}=$ standard deviation. ${ }^{\mathrm{b}}$ The mean Braden score is $>19$ at randomization, because most patients were surgical patients and became immobile after their operation. These patients were expected to develop a Braden score $<19$ after their surgical procedure. These patients did have a Prepurse score $\geq 20$ with an expected immobility of $\geq 3$ days and could therefore be included

\section{Cost-effectiveness}

The mean costs regarding preventive measures were $€ 47$ higher in the dressing group compared with the control group, although this difference is not significant. The higher costs were mainly a result of higher repositioning costs and costs of the dressing (Table 7.3). The cost effectiveness ratio to prevent one sacral pressure 
ulcer(category $1-3$ ) was $€ 2238$. Figure 7.2 shows that $76 \%$ of the bootstrapped ratio's lie within the quadrant where an increased effectiveness of the dressing strategy is achieved at higher costs compared to usual care. However, 21 percent of the ratio's lie within the inferior quadrant indicating that the dressings are less effective and more expensive. The acceptability curve (Figure 7.2) shows that the probability of the dressing strategy being cost-effective for the calculated ratio of $€ 2238$ is around $50 \%$.

Table 7.3 Cost-analysis of preventive measures

\begin{tabular}{lccc}
\hline & $\begin{array}{c}\text { Dressing group } \\
(\mathrm{n}=117)\end{array}$ & $\begin{array}{c}\text { Control group } \\
(\mathrm{n}=127)\end{array}$ & $\begin{array}{c}\text { Bootstrappedcost-difference } \\
(2.5 \text { th-97.5Cl) }\end{array}$ \\
\hline Repositioning costs & $€ 147$ & $€ 121$ & $€ 26(\mathrm{Cl}:-11 ; 69)^{\mathrm{b}}$ \\
Costs mattresses & $€ 2.92$ & $€ 2.20$ & $€ 0.5(\mathrm{Cl}: 0-3)^{\mathrm{b}}$ \\
Costs dressing & $€ 9.36^{\mathrm{a}}$ & $\mathrm{n} . \mathrm{a}$. & $€ 9.36$ \\
Total costs & $€ 160^{\mathrm{c}}$ & $€ 123$ euro $^{\mathrm{c}}$ & $€ 47(\mathrm{Cl}: 0-79)^{\mathrm{b}}$ \\
\hline
\end{tabular}

${ }^{a}$ Including 6 patients who did not receive any dressing and 31 patients who discontinued the intervention with the dressing prematurely. In accordance with the intention to treat analysis these patients were analysed in the intervention group. ${ }^{b}$ When zero is included in the confidence interval:cost-difference is not significant. ${ }^{c}$ Numbers do not exactly add up to the total because of the bootstrap analysis
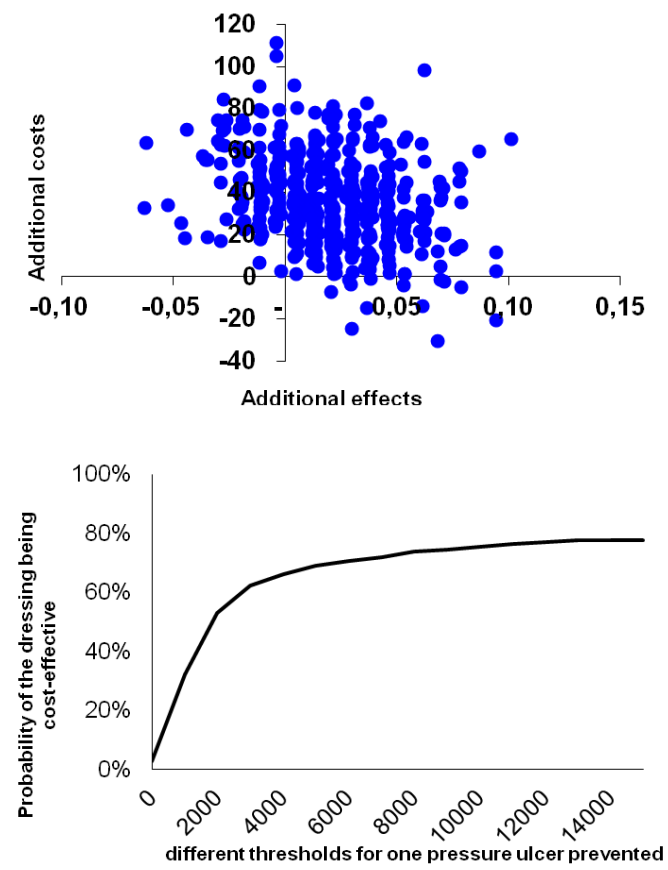

Figure 7.2 Cost-effectiveness plane and acceptability curve 


\section{Device deficiencies and adverse device effects (ADE-s)}

A total of 125 device deficiencies took place during this trial in 58 patients. Most of these device deficiencies consisted of curling of the dressing and it happened 115 times. In ten cases the dressing totally came off. In 24 patients the intervention was discontinued because the dressing kept on curling and the patients refused to wear the dressing any longer. A total of seven ADE-s took place in seven patients. Four patients developed blanchable erythema after the dressing was curled. Other ADE-s were the development of a rash (two patients) and the development of an intertrigo underneath the dressing (one patient) in these cases the intervention was discontinued.

\section{Discussion}

Our study showed that the use of a soft silicon sacral dressing is not a necessary addition to the current preventive pressure ulcer strategies in high-risk patients in a University hospital in the Netherlands. Because of the the low incidence of sacral pressure ulcers in this trial, however, we do not have enough power to conclude if this soft-silicon sacral dressing had any prophylactic effect.

Only 11 patients developed a sacral pressure ulcer: one patient in the treatment group developed a category 2 pressure ulcer a week after she refused to continue to wear the sacral dressing. The other patients in the dressing group developed a pressure ulcer underneath the dressing.

The incidence of sacral pressure ulcers $(4,5 \%)$ was lower than we expected at forehand. There are several explanations why the incidence of sacral pressure ulcers in this study was lower than we expected previously: One possible explanation is that we did not include patients admitted to the intensive care unit (ICU) and mentally disabled patients. Literature shows a higher incidence of pressure ulcers in the ICU. ${ }^{1,14}$ We did not focus on patients admitted to the ICU because we wanted to investigate the dressing in high-risk patients at other departments. We did not include mentally disabled patients because the Medical Ethics committee did not give permission to include these patients. The low incidence of pressure ulcers in this trial could also be explained because the attention on pressure ulcer prevention in our hospital increased during this study. With the use of a strict repositioning schedule and specialized matrasses, pressure ulcer preventive strategies are already quite developed and capable to reduce the incidence of sacral pressure ulcers in high-risk patients.

This study showed a high number of device deficiencies as the dressing curled in 62 patients. We decided to replace every curled dressing during the trial, because four patients developed blanchable erythema after the dressing was curled. Most of the patients in our trial were able to make slight changes in body position and this could 
possibly be a reason why the dressing curled. However, the curling also took place in totally immobile patients, so we cannot exclude the effect of external influences like: transfer from operating table to hospital bed, repositioning or daily care by general nurses. With the use of cavilon spray $(3 \mathrm{~m})$ to increase the adhesive capacities of the edges of the dressing, extra training for the research team by an expert from the dressing company and the removal of the dressing in patients who became totally mobile again, we undertook several measures to solve this problem but this did not result in less curling of the dressing.

Previously we expected lower median VAS scores for sacral pain and discomfort in the dressing group. These scores were low in the control group as well, which we see it as a confirmation of a well-developed pressure ulcer prevention strategy in our hospital. We chose the period day 3-8, because the mean period of immobility was $7.7 \pm 7.0$ days. Most patients were surgical patients, so they were not capable of giving VASscores at day two because that was the day of their surgery.

The cost difference between the dressing strategy and usual care was not significant but could have been higher if all patients in the dressing group would have received their dressing or the intervention with the dressing was not discontinued due to curling. The incremental cost effectiveness ratio of $€ 2238$ to prevent one pressure ulcer (category 1-3) was high compared with the weighted mean treatment costs of $€ 503$ for a pressure ulcer based on the study by Schuurman et al. ${ }^{12}$ In addition, the probability that the dressing is cost-effective for this amount is only $50 \%$. Hence, the dressing is not considered a cost-effective strategy.

The findings of our study differ from previous studies that reported promising results of the use of prophylactic wound dressings, including a soft silicon dressing. ${ }^{14,15,17,18}$ Most studies included only patients admitted to the emergency department or intensive care unit, did not mention the pressure ulcer categories, did not randomize patients and did not report on ADE-s and the cost effectiveness.

In a recent single-center randomized controlled trial, Santamaria et al. proved the effectiveness and cost-effectiveness of heel and sacral wound dressings in a ICU setting. Although, the incidence of sacral pressure ulcers was low as well, eight in the control group and two in the treatment group ( $n=440)$, the investigators are now using sacral dressings in all high-risk patients in their hospital. ${ }^{14,24}$ Therefore, the sacral dressing might be beneficial in settings where a higher incidence of hospital acquired pressure ulcers is expected.

Our trial had several limitations: First: it was performed in one hospital, so the results may not be generalizable to other hospitals or health care settings. We did not include mentally disabled patients or patients admitted to the ICU, so we cannot make any conclusions about these patient categories.

Another limitation is that the study was powered at 870 patients (435 in the dressing group and 435 in the control group) to find a $10 \%$ effect rate between groups. The effect of the dressing could never be of clinical importance in our setting, because of the low incidence of clinically relevant sacral pressure ulcers (category 2-4) in our trial. 
Therefore, the principal investigator decided to terminate the study prematurely. Although we included category 1 sacral pressure ulcers as a primary outcome as well, this category is mostly seen as an important warning sign and requires fewer clinical consequences. $^{25,26}$

The last limitation is that the research nurses were not blinded, because of the high visibility of the dressing, it was difficult to achieve this. This problem was partly solved by an independent wound care specialist who was called in to observe the research team when a pressure ulcer was suspected.

In conclusion, this trial does not prove that the use of a soft silicon sacral dressing is a necessary addition to current preventive pressure ulcer strategies in this setting. To make a reliable judgment about the clinical applicability of this dressing in other healthcare settings, further research should focus on a specific patient category with a high incidence of clinically relevant sacral pressure ulcers (category 2-4), and include enough patients to prove the effectiveness of this dressing without ignoring ADE-s and the cost-effectiveness. 


\section{References}

1. Keller BP, Wille J, van Ramshorst B, van der Werken C. Pressure ulcers in intensive care patients: a review of risks and prevention. Intensive Care Med. 2002;28(10):1379-1388.

2. Reddy M, Gill SS, Rochon PA. Preventing pressure ulcers: a systematic review. JAMA. 2006;296(8): 974-984.

3. European Pressure Ulcer Advisory Panel., National Pressure Ulcer Advisory Panel (U.S.). Prevention and treatment of pressure ulcers : clinical practice guideline : international guideline. Washington, D.C.: National Pressure Ulcer Advisory Panel; 2009.

4. Bansal C, Scott R, Stewart D, Cockerell CJ. Decubitus ulcers: a review of the literature. Int J Dermatol. 2005;44(10):805-810.

5. Mimura M, Ohura T, Takahashi M, Kajiwara R, Ohura N, Jr. Mechanism leading to the development of pressure ulcers based on shear force and pressures during a bed operation: influence of body types, body positions, and knee positions. Wound Repair Regen. 2009;17(6):789-796.

6. Schoonhoven L, Bousema MT, Buskens E, pre P-sg. The prevalence and incidence of pressure ulcers in hospitalised patients in the Netherlands: a prospective inception cohort study. Int J Nurs Stud. 2007;44(6):927-935.

7. Halfens RJ, Meesterberends E, van Nie-Visser NC, et al. International prevalence measurement of care problems: results. J Adv Nurs. 2013;69(9):e5-17.

8. Graves N, Birrell F, Whitby M. Effect of pressure ulcers on length of hospital stay. Infect Control Hosp Epidemiol. 2005;26(3):293-297.

9. Gorecki C, Brown JM, Nelson EA, et al. Impact of pressure ulcers on quality of life in older patients: a systematic review. J Am Geriatr Soc. 2009;57(7):1175-1183.

10. Chou R, Dana T, Bougatsos C, et al. Pressure ulcer risk assessment and prevention: a systematic comparative effectiveness review. Ann Intern Med. 2 2013;159(1):28-38.

11. Defloor T, De Bacquer D, Grypdonck MH. The effect of various combinations of turning and pressure reducing devices on the incidence of pressure ulcers. Int J Nurs Stud. 2005;42(1):37-46.

12. Schuurman JP, Schoonhoven L, Defloor T, van Engelshoven I, van Ramshorst B, Buskens E. Economic evaluation of pressure ulcer care: a cost minimization analysis of preventive strategies. Nurs Econ. 2009;27(6):390-400, 415.

13. Vanderwee K, Grypdonck MH, De Bacquer D, Defloor T. Effectiveness of turning with unequal time intervals on the incidence of pressure ulcer lesions. J Adv Nurs. 2007;57(1):59-68.

14. Santamaria N, Gerdtz M, Sage S, et al. A randomised controlled trial of the effectiveness of soft silicone multi-layered foam dressings in the prevention of sacral and heel pressure ulcers in trauma and critically ill patients: the border trial. Int Wound J. 2015;12(3):302-308.

15. Chaiken N. Reduction of sacral pressure ulcers in the intensive care unit using a silicone border foam dressing. J Wound Ostomy Continence Nurs. 2012;39(2):143-145.

16. Ohura N, Ichioka S, Nakatsuka T, Shibata M. Evaluating dressing materials for the prevention of shear force in the treatment of pressure ulcers. J Wound Care. 2005;14(9):401-404.

17. Brindle CT, Wegelin JA. Prophylactic dressing application to reduce pressure ulcer formation in cardiac surgery patients. J Wound Ostomy Continence Nurs. 2012;39(2):133-142.

18. Walsh NS, Blanck AW, Smith L, Cross M, Andersson L, Polito C. Use of a sacral silicone border foam dressing as one component of a pressure ulcer prevention program in an intensive care unit setting. J Wound Ostomy Continence Nurs. 2012;39(2):146-149.

19. Moore ZE, Webster J. Dressings and topical agents for preventing pressure ulcers. Cochrane Database Syst Rev. 2013;8:CD009362.

20. Moher D, Schulz KF, Altman DG. The CONSORT statement: revised recommendations for improving the quality of reports of parallel-group randomised trials. Lancet. 2001;357(9263):1191-1194.

21. Schoonhoven L, Grobbee DE, Donders AR, et al. Prediction of pressure ulcer development in hospitalized patients: a tool for risk assessment. Qual Saf Health Care. 2006;15(1):65-70.

22. Hakkart- van- Roijen L, Tan SS, Bouwmans CAM. Handleiding voor kostenonderzoek. College voor zorgverzekeringen. 2010.

23. Efron B, Tibshirani R. An introduction to the bootstrap. New York: Chapman \& Hall; 1993. 
24. Santamaria N, Liu W, Gerdtz M, et al. The cost-benefit of using soft silicone multilayered foam dressings to prevent sacral and heel pressure ulcers in trauma and critically ill patients: a within-trial analysis of the Border Trial. Int Wound Journal. 2015;12(3):344-350.

25. Vanderwee K, Grypdonck M, Defloor T. Non-blanchable erythema as an indicator for the need for pressure ulcer prevention: a randomized-controlled trial. J Clin Nurs. 2007;16(2):325-335.

26. Halfens RJ, Bours GJ, Van Ast W. Relevance of the diagnosis 'stage 1 pressure ulcer': an empirical study of the clinical course of stage 1 ulcers in acute care and long-term care hospital populations. J Clin Nurs. 2001;10(6):748-757. 



\section{Chapter 8}

General discussion 

Prevention of pressure ulcers remain an important healthcare challenge. The goal of this thesis was to gain insight in the pathophysiology and to improve the prevention of pressure ulcers.

\section{Predicting pressure ulcer risk in patients}

Identifying patients at risk is the first step in effective prevention. Nowadays, pressure ulcer risk assessment is primarily based on risk assessment scales. Most of these scales are based on expert opinion and are therefore subjective, which limit its use in clinical practice ${ }^{1-3}$. Schoonhoven and colleagues ${ }^{1}$ demonstrated in a large prospective cohort study that the use of the most commonly used risk assessment scales, i.e. the Norton scale, The Braden and Waterlow scale, would lead to ineffective use of preventive measures. In contrast to subjective risk assessment scales, biomarkers are by definition objective and can be measured accurately ${ }^{4}$. Earlier studies have shown that differences in the cutaneous microbiome is associated with several skin diseases ${ }^{5-8}$, offering a potential tool for predicting disease risk in patients. In chapter 2, the cutaneous microbiome was assessed as a potential new biomarker to identify patients at risk for the development of pressure ulcers. Before a biomarker can be used to predict disease risk in patients, more insight has to be obtained about the microbiome in the healthy state and disease state. Therefore, the microbiome of the unaffected skin was investigated in hospitalized patients with sacral pressure ulcers compared with hospitalized patients without sacral pressure ulcers. Results of this study demonstrated that the microbiome of the unaffected skin from patients with sacral pressure ulcers differs with those without pressure ulcers. The abundance of 23 species was significantly between patients with and without pressure ulcers. A random Forest analysis was carried out to determine the clinical relevance of bacterial species and clinical factors in patients with pressure ulcers. Interestingly, clinical factors were not associated with pressure ulcer status, but classification based on bacterial species abundance, showed that eight species are related to pressure ulcer status and predicted pressure ulcer status in $81 \%$ of the patients. In line herewith, a multivariate approach leads to an improved classification and is more robust and more prognostic than a single species. Before the cutaneous microbiome could be implemented as a new biomarker for predicting pressure ulcer risk, a prospective cohort study need to be carried out to investigate if the cutaneous microbiome differs before the presence of a pressure ulcer. A next step would validate this method as a new prediction tool and investigate its generalisability in different hospitals. Additionally, it could lead to targeted preventive interventions too, by modulating the cutaneous microbiome in a way that is beneficial for the host. 


\section{Effect of shear and pressure on skin viability}

Since prolonged mechanical loading on the skin is considered to be the most important factor in the development of pressure ulcers ${ }^{9-11}$, effort should be made to reduce its harmful effect on skin viability. Most pressure ulcers develop over a bony prominence, because the underlying tissues are thin resulting in high mechanical loading and increased tissue deformation. The importance of pressure in the development of pressure ulcers is well known and with the help of pressure mapping many devices have been developed to minimise the amount of pressure on skin $^{12}$. It was Reiche ${ }^{13}$ who described the importance of shear in the development of pressure ulcers as opposed to pressure alone in 1958. This hypothesis was supported by Dinsdale $^{14}$, who demonstrated in a swine model that less pressure was needed to cause a pressure ulcer when shear was added. In contrast to pressure, reliable measurement of shear remains a difficult challenge. Although it is hard to measure the amount of shear in skin, it is possible to measure the effect of mechanical loading on skin viability. Only a few studies investigated the effect of shear on skin viability $^{15-17}$. Especially, the two studies of Manorama and colleagues ${ }^{15,16}$ cannot be considered reproducible, as they used a magnitude of shear which was induced by the test person itself, leading to a wide range in the magnitude of shear between the different test persons.

To investigate the effect of shear on skin viability and test shear reducing capacities of materials, a new model was developed and validated in chapter 3. In 10 healthy participants to investigate the response of shear combined with pressure on skin viability by analysing a non- invasive biomarker and the hyperaemic response. A loading of $3.9 \mathrm{kPa}$ of pressure or $14.5 \mathrm{~N}$ of shear combined with $2.4 \mathrm{kPa}$ of pressure was applied for 15 or 30 minutes respectively on the volar aspect of the forearm. IL-1 $\alpha$ is a damage associated molecular pattern (DAMP) and essential in the initiation of inflammatory skin response after disruption of the skin barrier ${ }^{18,19}$. Furthermore, it is stored in preformed pools in the healthy keratinocytes and therefore immediately released when the keratinocytes are damaged and can be collected noninvasively with the use of Sebutape ${ }^{20}$. After 30 minutes of loading, the results demonstrated that when shear is added to pressure, the increase of normalized IL- $1 \alpha /$ TP- ratio was significantly higher compared with pressure loading. This implies that in the presence of shear, more keratinocytes becomes damaged, resulting in an upregulation of IL-1 $\alpha$. Post-load reactive hyperaemia represents also an important response once mechanical loading on the skin is relieved ${ }^{21,22}$. It occurs as a reaction on tissue ischemia, deformation and the accumulation of metabolic waste products. In this study, the reactive hyperaemic response was measured with a Laser Doppler and expressed as cutaneous blood cell flux (CBF) in arbitrary units. The reactive hyperaemic response was significantly increased when shear was added to pressure. The results of this study suggest that when shear is added to pressure, the skin is more likely to become damaged, tissue becomes more ischemic and there is an 
increase in tissue deformation. This study only included young healthy volunteers, so the result of cannot be directly extrapolated to patients at risk for pressure ulcers who are more frequently of old age, critically ill, have multiple comorbidities and bound to a bed or chair ${ }^{23-25}$.

For example, patients with type 2 Diabetes Mellitus (DM) are at increased risk for the development of chronic wounds such as pressure ulcers or diabetic foot ulcers, because functional abnormalities lead to increased pressures and several reparative responses are impaired ${ }^{26}$. Although plantar shear is associated with increased risk for foot ulceration ${ }^{27-29}$, little is known about the diabetic skin response to shear and the influence of diabetic neuropathy on this response. In chapter 4, the shear model was used to investigate the immunological and reactive hyperaemic skin response of patients with type $2 \mathrm{DM}$ with and without neuropathy. In 10 patients with type $2 \mathrm{DM}$ without neuropathy, 10 patients with type 2 DM with neuropathy and 10 healthy volunteers, the skin of the forearm was loaded with shear combined with pressure, and only pressure for 30 minutes. The results of this study demonstrate that the reactive hyperaemic response and IL-1 $\alpha$ release was increased after it was loaded with shear (combined with pressure) in all the groups, and was higher after it was loaded with pressure alone. The reactive hyperaemic response was decreased in patients with type $2 \mathrm{DM}$, suggesting that in type $2 \mathrm{DM}$ the microcirculatory (reparative) responses are impaired, resulting in impaired damage control once the skin is loaded with shear combined with pressure. In earlier studies, the reactive hyperaemic response on several stimuli was also impaired and is probably related decreased endothelial dependent and independent vasodilation ${ }^{30-33}$. The reactive hyperaemic response in chapter 4 was negatively correlated with blood glucose values, suggesting that short-term metabolic control is also important in protecting the skin against external noxious stimuli. In contrast to earlier studies, which investigated the microvascular response to loading ${ }^{30,34}$, the presence of diabetic polyneuropathy was not associated with a decreased reactive hyperaemic skin response to shear. Therefore, the inability to sense excessive shear loading on skin could possibly be a main factor in the development skin damage in patients with diabetic polyneuropathy.

Another group of patients at risk for the development of pressure ulcers are trauma patients, because spinal immobilisation is a common practice in these patients to maintain spinal alignment and protect the spinal cord from injury ${ }^{35}$. The spine board is a commonly used device to transfer patients from the scene of trauma to the hospital. However, prolonged immobilization on its rigid surface is associated with pain and discomfort $^{36,37}$, but also increase tissue deformation ${ }^{38}$ and the risk of developing pressure ulcers ${ }^{39}$. Chapter $\mathbf{5}$ is a prospective, randomised open label crossover study that examined the relationship between tissue interface pressure (measured with a pressure mapping device), and the response of skin, in healthy participants on two types of spine boards. The skin response, i.e. II-1 $\alpha /$ TP-ratio, lactate and skin redness, of twelve healthy participants were assessed when lying on a rigid and soft-layered 
spine board for one hour. Measuring tissue interface pressure with pressure mapping is common practice to determine if support devices are safe to protect the skin against pressure injury. Mostly, a cut off score of $32 \mathrm{mmHg}$ is used, because the hydrostatic pressure of capillaries is $32 \mathrm{mmHg}^{12,40}$. If pressure exceeds $32 \mathrm{mmHg}$, it is believed that capillaries become occluded and ischemia/ hypoxia of skin tissue occurs, which eventually could lead to pressure injury. Therefore, it is assumed that if tissue interface pressure can be kept below $32 \mathrm{mmHg}$, it will be safe to prevent the development of pressure ulcers in patients ${ }^{41-43}$. However, as reported by several recent ex vivo studies ${ }^{9,44,45}$, the cut off score of $32 \mathrm{mmHg}$ does not correlate with actual cell death, because tissue injury is mainly the direct result of internal tissue deformation. When internal tissue deformation becomes high, it will lead to cell death and the release of cytokines such as IL-1 $\alpha$. This is confirmed by the results presented in chapter 5: Although tissue interface pressures were higher on the rigid spine board compared to the values on the soft-layered spine board, it was not associated with significantly differences in IL-1 $\alpha$ release and post-load lactate concentrations. In addition, both IL-1 $\alpha /$ TP-ratio and lactate concentrations were strongly correlated with pressure exposure time, suggesting that minimise the time of mechanical loading could be more important in the prevention of skin damage than the reducing the amount of tissue interface pressure per se.

\section{Clinical implications}

Besides pressure, shear is considered to also be a major factor in the development of pressure ulcers ${ }^{13,17,46,47}$, but it remains a major challenge to reduce its harmful effects on skin. It is believed that the prophylactic application of wound dressings between the skin and the support surface can protect the skin against injury by reducing tissue deformation by absorbing both pressure and shear ${ }^{48,49}$. Several studies already demonstrated the shear reducing capacities of wound dressings, however, these studies have not been performed in humans ${ }^{50-52}$. Since then, a variety of dressing manufacturers claims that their dressings are effective in the prevention of pressure ulcers (mostly without evidence), just by assuming that their dressings are just as effective as other dressings.

In chapter 6, the newly validated shear model was used to investigate the shear reducing capacities of three commercially available foam dressings in humans. These dressing were chosen, because we wanted to investigate if multi-layered dressing perform better than single-layered dressings. In this study, we compared a singlelayered foam dressing, a two-layered dressing and a three-layered foam dressing. After a combined loading of shear combined with pressure for 30 minutes, a decrease in post-load IL-1 $\alpha$ release (used as a measure of tissue damage) was measured when a dressing was applied between the skin and the indenter. Interestingly, only the multilayered dressings were able to reduce the amount of post-load reactive hyperaemia 
and the multi layered dressings performed better than the single layered dressings in general. This is in line with an earlier observation of Levy and colleagues ${ }^{49}$ based on a theoretical finite element analysis. They predicted a decrease in tissue deformation when a multi-layered dressing was applied to the skin, because multi-layered dressings are more capable to absorb shear in comparison to a single layered dressing. In our model, it seems that foam dressings, especially those with a multi-layered design, could be beneficial in pressure ulcer preventive strategies. A comment need to be placed by the interpretation of the results demonstrated in chapter 6 . Because dressings from different manufacturers were used, it is hard to determine if other factors influence the shear reducing capacities of dressings as well. It must be noted that that shear-reducing capacities could be dependent on other factors including material stiffness, material thickness, chemistry of contact layer could influence as well and this must be considered in future studies. For healthcare workers, it is important to notice that differences in performance occur between dressings from different manufacturers. In our opinion, an optimal dressing design includes multiple layers, a low frictional outer contact layer and have enough strength to adhere to the patients skin to remain in place in a clinical setting. However, more research should be carried out to select the best materials, and engineer the best dressing construction to improve the protective mechanism of prophylactic dressings. In order to answer the question if dressings are also effective in protecting the skin of diabetic, elderly or malnourished patients, they should be tested in different patient groups.

Other than basic research, several clinical studies demonstrate that wound dressings could be effective as an addition to already existing pressure ulcer preventive strategies. This was especially investigated in patients admitted to the intensive care unit (ICU) $)^{53-57}$.

A clinical randomised controlled trial was initiated in the Maastricht University Medical Centre (chapter 7) to investigate if the use of a soft silicone dressing could prevent the development of sacral pressure ulcers in high risk hospitalised patients who were not admitted to the ICU. Newly admitted patients with a Braden score $<19^{1}$ or a Prepurse score $\geq 21^{58}$ were able to participate. The study was originally powered at 870 patients, but was prematurely terminated after a total of 244 patients were included, because of the low incidence of sacral pressure ulcers in this trial and high number of device deficiencies. Only 11 patients developed a sacral pressure ulcer, four in the dressing group (3.4\%) and seven in the control group (5.3\%), four patients developed a pressure ulcer $\geq$ category 2 . This underlines the fact that risk assessment scales are not effective in predicting pressure ulcer risk and leads to ineffective use of preventive measures. In addition to the low incidence of sacral pressure ulcers, a high number of device deficiencies were observed, because the dressing did not stay in place and had to be removed early in 62 patients of the 117 patients who received a dressing. Possibly, the patients in our study group were too mobile in comparison to the mostly immobile patients of the ICU, although curling of the dressing also occurred in totally immobile patients as well. Another point of concern was the fact 
that the cost effectiveness ratio to prevent one sacral pressure ulcer was $€ 2238$ with the dressing strategy, while the mean treatment cost of a pressure ulcers is predicted to be only $€ 503^{59}$. Hence, the dressing strategy was not considered effective in this clinical setting, so we decided to prematurely terminate the study with this set-up and materials used.

A few points needs to be addressed before a reliable judgment about the clinical applicability of prophylactic dressing use in high risk hospitalised patients who are not admitted to the ICU can be made. First, future research should focus on a high risk patient category with a high incidence of clinical relevance sacral pressure ulcers ( $\geq$ category 2). For example, a Belgian study ${ }^{60}$ compared the Braden scale with the use of non-blanchable erythema (category 1 pressure ulcer). They proved that fewer patients needed preventive measures when the appearance of non-blanchable erythema was used to allocate preventive measures without increasing the incidence of clinical relevant pressure ulcers. Second, the adherence strength of the dressing should be strong enough to hold the dressing in place in these patients. Finally, it is important to analyse whether the relationship between the clinical outcomes and total costs are acceptable to help healthcare decision makers to allocate their financial resources efficiency.

\section{Concluding remarks}

This thesis offers insights in the prevention of pressure ulcers by focussing on the factor shear, the use of prophylactic dressings and the cutaneous microbiome in the development of pressure ulcers. It demonstrates that the cutaneous microbiome offers a potential new tool in predicting pressure ulcer risk in patients. The factor shear is important in the development of pressure ulcers and the skin response to shear can be measured by analysing IL-1 $\alpha$ and reactive hyperaemic parameter. The reparative response to shear is impaired in patients with type 2 DM making them more susceptible for impaired damage control.

Foam dressings can reduce the harmful effect of shear on skin viability, especially those with a multi-layered design. However, when initiating a trial to prove the efficacy of prophylactic dressing in a clinical setting, researchers should focus on a patient group with a high incidence of pressure ulcers and report on patient related experiences, adverse device effects and economic evaluations. Then, other healthcare workers can make a reliable judgment about the clinical applicability of prophylactic dressings. 


\section{References}

1. Schoonhoven L, et al. Prospective cohort study of routine use of risk assessment scales for prediction of pressure ulcers. BMJ. 2002;325(7368):797.

2. Chou $\mathrm{R}$, et al. Pressure ulcer risk assessment and prevention: a systematic comparative effectiveness review. Ann Intern Med. 2013;159(1):28-38.

3. Reddy M, Gill SS. The effectiveness of pressure ulcer risk assessment instruments and associated intervention protocols remains uncertain. Evid Based Med. 2014;19(3):93.

4. Strimbu K, Tavel JA. What are biomarkers? Curr Opin HIV AIDS. 2010;5(6):463-466.

5. Findley K, Grice EA. The skin microbiome: a focus on pathogens and their association with skin disease. PLoS Pathog. 2014;10(10):e1004436.

6. Fitz-Gibbon S, et al. Propionibacterium acnes strain populations in the human skin microbiome associated with acne. J Invest Dermatol. 2013;133(9):2152-2160.

7. Ganju P, et al. Microbial community profiling shows dysbiosis in the lesional skin of Vitiligo subjects. Sci Rep. 2016;6:18761.

8. Horton JM, et al. The cutaneous microbiome in outpatients presenting with acute skin abscesses. J Infect Dis. 2015;211(12):1895-1904.

9. Oomens CW, et al. Pressure induced deep tissue injury explained. Ann Biomed Eng, 2015;43(2): 297-305.

10. Stekelenburg A, et al. Deep tissue injury: how deep is our understanding? Arch Phys Med Rehabil. 2008;89(7):1410-1413.

11. Hoogendoorn I, et al. The effect of pressure and shear on tissue viability of human skin in relation to the development of pressure ulcers: a systematic review. J Tissue Viability 2017;26(3):156-171.

12. Swain ID, Bader D:. The measurement of interface pressure and its role in soft tissue breakdown. J Tissue Viability. 2002;12(4):132-134, 136-137, 140-146.

13. Reichel SM. Shearing force as a factor in decubitus ulcers in paraplegics. J Am Med Assoc. 1958; 166(7):762-763.

14. Dinsdale SM. Decubitus ulcers: role of pressure and friction in causation. Arch Phys Med Rehabil. 1974;55(4):147-152.

15. Manorama, A.A., et al., Blood perfusion and transcutaneous oxygen level characterizations in human skin with changes in normal and shear loads--implications for pressure ulcer formation. Clin Biomech (Bristol, Avon). 2010;25(8):823-828.

16. Manorama A, et al. Quantifying the effects of external shear loads on arterial and venous blood flow: implications for pressure ulcer development. Clin Biomech (Bristol, Avon). 2013;28(5):574-578.

17. Goossens RH, et al. Influence of shear on skin oxygen tension. Clin Physiol. 1994;14(1):111-118.

18. Suwara MI, et al. IL-1alpha released from damaged epithelial cells is sufficient and essential to trigger inflammatory responses in human lung fibroblasts. Mucosal Immunol. 2014;7(3):684-693.

19. Wood LC, et al. Barrier disruption stimulates interleukin-1 alpha expression and release from a preformed pool in murine epidermis. J Invest Dermatol. 1996;106(3):397-403.

20. Perkins $\mathrm{MA}$, et al. A noninvasive method to assess skin irritation and compromised skin conditions using simple tape adsorption of molecular markers of inflammation. Skin Res Technol. 2001;7(4): 227-237.

21. Bliss MR. Hyperaemia. J Tissue Viability. 1998;8(4):4-13.

22. Liao F, Burns S, Jan YK. Skin blood flow dynamics and its role in pressure ulcers. J Tissue Viability. 2013;22(2):25-36.

23. Allman RM. Pressure ulcers among the elderly. N Engl J Med. 1989;320(13):850-853.

24. Bansal C, et al. Decubitus ulcers: a review of the literature. Int J Dermatol. 2005;44(10):805-810.

25. Coleman $\mathrm{S}$, et al. Patient risk factors for pressure ulcer development: systematic review. Int J Nurs Stud. 2013;50(7):974-1003.

26. Schaper NC, Havekes B. Diabetes: impaired damage control. Diabetologia. 2012;55(1):18-20.

27. Lord M, Hosein R. A study of in-shoe plantar shear in patients with diabetic neuropathy. Clin Biomech (Bristol, Avon). 2000;15(4):278-283.

28. Hamatani M, et al. Factors Associated With Callus in Patients with Diabetes, Focused on Plantar Shear Stress During Gait. J Diabetes Sci Technol. 2016;10(6):1353-1359. 
29. Yavuz M, et al. Peak Plantar Shear and Pressure and Foot Ulcer Locations: A Call to Revisit Ulceration Pathomechanics. Diabetes Care. 2015;38(11):e184-e185.

30. Nabuurs-Franssen $\mathrm{MH}$, et al. The effect of polyneuropathy on foot microcirculation in Type II diabetes. Diabetologia. 2002;45(8):1164-1171.

31. Johnstone $\mathrm{MT}$, et al. Impaired endothelium-dependent vasodilation in patients with insulindependent diabetes mellitus. Circulation. 1993;88(6):2510-2516.

32. Jan YK, et al. Skin blood flow response to locally applied mechanical and thermal stresses in the diabetic foot. Microvasc Res. 2013;89:40-46.

33. Fromy B, et al. Early decrease of skin blood flow in response to locally applied pressure in diabetic subjects. Diabetes. 2002;51(4):1214-1217.

34. Hsu PC, et al. Nailfold capillary abnormalities are associated with type 2 diabetes progression and correlated with peripheral neuropathy. Medicine (Baltimore). 2016;95(52):e5714.

35. Ahn $\mathrm{H}$, et al. Pre-hospital care management of a potential spinal cord injured patient: a systematic review of the literature and evidence-based guidelines. J Neurotrauma. 2011;28(8):1341-1361.

36. Chan D, et al. The effect of spinal immobilization on healthy volunteers. Ann Emerg Med. 1994;23(1): 48-51.

37. Cordell WH, et al. Pain and tissue-interface pressures during spine-board immobilization. Ann Emerg Med. 1995;26(1):31-36.

38. Oomens $\mathrm{CW}$, et al. A numerical study to analyse the risk for pressure ulcer development on a spine board. Clin Biomech (Bristol, Avon). 2013;28(7):736-742.

39. Ham W, et al. Pressure ulcers from spinal immobilization in trauma patients: a systematic review. J Trauma Acute Care Surg. 2014;76(4):1131-1141.

40. Bouten CV, et al. The etiology of pressure ulcers: skin deep or muscle bound? Arch Phys Med Rehabil, 2003;84(4):616-619.

41. Lyder $\mathrm{CH}$, Ayello EA. Pressure Ulcers: A Patient Safety Issue, in Patient Safety and Quality: An Evidence-Based Handbook for Nurses, R.G. Hughes, Editor. 2008: Rockville (MD).

42. Ros-Mar R, Martinez-Gamarra M. The effect of a double-regression hospital bed on interface pressure. Appl Nurs Res. 2011;24(4):e79-85.

43. Peterson MJ, et al. Effects of turning on skin-bed interface pressures in healthy adults. J Adv Nurs. 2010;66(7):1556-1564.

44. Linder-Ganz E, Gefen A. The effects of pressure and shear on capillary closure in the microstructure of skeletal muscles. Ann Biomed Eng. 2007;35(12):2095-2107.

45. Gefen A, et al. Strain-time cell-death threshold for skeletal muscle in a tissue-engineered model system for deep tissue injury. J Biomech. 2008;41(9):2003-2012.

46. Zhang $M$, Roberts VC. The effect of shear forces externally applied to skin surface on underlying tissues. J Biomed Eng. 1993;15(6):451-456.

47. Bennett $L$, et al. Shear vs pressure as causative factors in skin blood flow occlusion. Arch Phys Med Rehabil. 1979;60(7):309-314.

48. Gefen A, Kottner J, Santamaria N. Clinical and biomechanical perspectives on pressure injury prevention research: The case of prophylactic dressings. Clin Biomech (Bristol, Avon). 2016;38:29-34.

49. Levy A, Frank MB, Gefen A. The biomechanical efficacy of dressings in preventing heel ulcers. J Tissue Viability. 2015;24(1):1-11.

50. Call $E$, et al. Enhancing pressure ulcer prevention using wound dressings: what are the modes of action? Int Wound J. 2015;12(4):408-413.

51. Ohura T, Takahashi M, Ohura N Jr. Influence of external forces (pressure and shear force) on superficial layer and subcutis of porcine skin and effects of dressing materials: are dressing materials beneficial for reducing pressure and shear force in tissues? Wound Repair Regen. 2008;16(1):102-107.

52. Ohura N, et al. Evaluating dressing materials for the prevention of shear force in the treatment of pressure ulcers. J Wound Care. 2005;14(9):401-404.

53. Santamaria N, et al. A randomised controlled trial of the effectiveness of soft silicone multi-layered foam dressings in the prevention of sacral and heel pressure ulcers in trauma and critically ill patients: the border trial. Int Wound J. 2015;12(3):302-308.

54. Brindle CT, Wegelin JA. Prophylactic dressing application to reduce pressure ulcer formation in cardiac surgery patients. J Wound Ostomy Continence Nurs. 2012;39(2):133-142. 
55. Chaiken N. Reduction of sacral pressure ulcers in the intensive care unit using a silicone border foam dressing. J Wound Ostomy Continence Nurs. 2012;39(2):143-145.

56. Kalowes P, Messina V, Li M. Five-Layered Soft Silicone Foam Dressing to Prevent Pressure Ulcers in the Intensive Care Unit. Am J Crit Care. 2016;25(6): e108-e119.

57. Santamaria N, et al. Clinical effectiveness of a silicone foam dressing for the prevention of heel pressure ulcers in critically ill patients: Border II Trial. J Wound Care. 2015;24(8):340-345.

58. Schoonhoven L, et al. Prediction of pressure ulcer development in hospitalized patients: a tool for risk assessment. Qual Saf Health Care. 2006;15(1):65-70.

59. Schuurman JP, et al. Economic evaluation of pressure ulcer care: a cost minimization analysis of preventive strategies. Nurs Econ. 2009;27(6):390-400, 415.

60. Vanderwee K, Grypdonck M, Defloor T. Non-blanchable erythema as an indicator for the need for pressure ulcer prevention: a randomized-controlled trial. J Clin Nurs. 2007;16(2):325-335. 

Nederlandse samenvatting 



\section{Nederlandse samenvatting}

De ontwikkeling van decubitus, beter bekend als doorligwonden of drukplekken, wordt gezien als een groot probleem binnen allerlei zorginstellingen. Decubitus is geassocieerd met veel pijn, lagere kwaliteit van leven, een verhoogde morbiditeit en mortaliteit. Het doel van dit proefschrift was dan ook om meer inzicht te vergaren in de pathofysiologie en biomechanica van het ontstaan van decubitus. Deze kennis hebben we gebruikt om materialen te testen die mogelijk decubitus kunnen voorkomen.

Hoofdstuk 1 bevat de algemene introductie en hierin worden de hoofdlijnen van dit proefschrift gepresenteerd.

In hoofdstuk 2 presenteren we een studie waarbij we hebben onderzocht of er verschillen bestaan tussen het microbioom van de niet aangedane huid tussen patiënten met decubitus op het sacrum en patiënten zonder decubitus. Bij 30 patiënten, 15 met decubitus op het sacrum en 15 zonder decubitus, hebben we via 16s rRNA sequencing het microbioom van de huid bepaald. De resultaten van deze studie toonden aan dat er verschillen bestaan in het microbioom van de huid tussen de twee patiëntengroepen. Deze studie liet zien dat de hoeveelheid van 23 soorten bacteriën significant verschillend zijn tussen deze twee groepen. Met name Staphylococcus Aureus en een niet geclassificeerde Enterococcus kwamen meer voor op de huid van patiënten met sacrale decubitus. Daarnaast bleek uit een Random Forest analyse dat we met een combinatie van acht soorten de aanwezigheid van decubitus (wel/niet) in $81 \%$ van de gevallen correct konden voorspellen. Concluderend bestaat er een verschil in microbioom tussen patiënten met en zonder decubitus op het sacrum. Ook bleek dat een multivariate benadering beter voorspellend was dan een benadering gericht op een enkele soort. Deze resultaten kunnen in de toekomst mogelijk gebruikt worden om het risico op decubitus beter te voorspellen. Hierbij zou het microbioom als eventuele biomarker gebruikt kunnen worden.

Decubitus wordt veroorzaakt door mechanische belasting van de huid door middel van druk en shear force. De schadelijke effecten van shear force in de huid waren moeilijk meetbaar, omdat er geen betrouwbare methode bestond die de invloed van shear force in de huid weergeeft. Hierdoor zijn er vrijwel geen materialen ontwikkeld die specifiek de nadelige effecten van shear force kunnen reduceren. Daarom hebben we in hoofdstuk 3 een model ontwikkeld en gevalideerd waarmee we op een gecontroleerde manier shear force kunnen toedienen op de huid van gezonde proefpersonen. Een mechanische belasting van $3.9 \mathrm{kPa}$ druk en $14.5 \mathrm{~N}$ shear force in combinatie met $2.4 \mathrm{kPa}$ druk werd toegediend op de huid van de arm bij tien gezonde mannen. Het effect hiervan hebben we gemeten door middel van de IL-1a/TP-ratio en 
de reactieve hyperaemische huid respons. II-1 $\alpha$ is een cytokine dat ligt opgeslagen in de huidcellen en vrijkomt indien deze cellen beschadigd raken. Hiermee wordt een immuunreactie opgewekt die uiteindelijk zal lijden tot regeneratie van nieuwe huidcellen. De reactieve hyperaemische respons is een toename in doorbloeding in de huid nadat deze mechanisch is belast. Het ontstaat ten gevolge van weefsel deformatie en ischemie en wordt gezien als een beschermende reactie om ischemie te herstellen en metabolieten te verwerken. Onze resultaten tonen aan dat de IL-

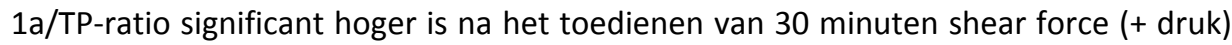
in vergelijking met alleen het toedienen van druk. Daarnaast zagen we dat de toename van reactieve hyperaemische respons na het 15 minuten toedienen van shear force (+ druk) significant hoger is in vergelijking met het alleen toedienen van druk. Concluderend laat deze studie zien dat zowel de IL-1a/TP-ratio, als de reactieve hyperaemische respons gebruikt kunnen worden als maat voor effect van shear force in de huid. Zodoende kan onze methode gebruikt worden om de effecten van shear force in de huid te bestuderen en kunnen er materialen mee onderzocht worden die de nadelige effecten van shear force in de huid kunnen reduceren.

In hoofdstuk 4 hebben wij het shear model gebruikt om de immunologische en reactieve hyperaemische respons van de huid te onderzoeken in patiënten met diabetes mellitus (DM) type 2 met en zonder polyneuropathie (PNP). Hiervoor werd de huid 30 minuten belast met shear force (+ druk) en alleen druk in tien patiënten met DM type 2 zonder PNP, tien patiënten met DM type 2 met PNP en tien gezonde vrijwilligers. De IL-1 $\alpha$ concentratie en reactieve hyperaemische respons was significant gestegen in alle drie de groepen na het belasten van de huid met shear force (+ druk). Ook was deze stijging hoger vergeleken met de stijging van IL-1 $\alpha$ en reactieve hyperaemie na het toedienen van alleen druk op de huid.

De reactieve hyperaemische reactie was verminderd in patiënten met DM type 2 vergeleken met de gezonde vrijwilligers. Dit suggereert dat in type 2 DM de (beschermende) microcirculatoire respons is afgenomen, waardoor het natuurlijke herstel na mechanische belasting van de huid met shear force+ druk is verminderd. Daarnaast toonde dit onderzoek ook aan dat glucose concentraties in het bloed negatief gecorreleerd waren met de reactieve hyperaemische reactie na het toedienen van shear force. Dit zou kunnen inhouden dat het reguleren van glucose concentratie ook op korte termijn invloed kan hebben op het ontstaan van wonden.

De harde wervelplank wordt buiten het ziekenhuis gebruikt om patiënten met verdenking van wervelletsel te transporteren. Echter, veroorzaakt deze plank veel pijn en ongemak en kan zelfs tot de ontwikkeling van decubitus leiden. In Hoofdstuk $\mathbf{5}$ beschrijven wij een onderzoek waarbij twaalf gezonde vrijwilligers een uur lang op een harde wervelplank en een zachte wervelplank hebben gelegen. Gedurende dit uur is de druk en huidreactie bepaald op het sacrum. Uitkomstmaten waren tissue interface druk, II-1 $\alpha$ /TP-ratio, lactaat concentratie en roodheid van de huid. De 
resultaten toonde aan dat het vrijkomen van IL-1 $\alpha$ gerelateerd was aan duur van de druk, maar niet aan de hoeveelheid druk die werd toegediend. Hieruit blijkt dat ook de duur waaraan iemand aan druk wordt blootgesteld belangrijk is in het voorkomen van weefselschade en dus tot een minimum beperkt dient te worden.

In Hoofdstuk 6 hebben wij met het shear model kunnen aantonen dat drie commercieel verkrijgbare foam dressings de effecten van shear force in de huid kunnen reduceren. Hiervoor hebben wij drie verschillende foam dresisngs gebruikt: één dressing met één laag, één dressing met twee lagen en één dressing met drie lagen. Het vrijkomen van IL-1 $\alpha$ en de reactieve hyperaemische respons was significant verminderd indien een dressing met meerdere lagen werd gebruikt in vergelijking met de dressing met één laag. Hieruit kunnen wij concluderen dat foam dressings de effecten van shear force kunnen reduceren en dat de meerlaagse dressings hierin het meest effectief bleken. Mogelijk kunnen deze dressings dus van toegevoegde waarde zijn in het voorkomen van decubitus.

Hoofdstuk 7 laat de data zien van een gerandomiseerde gecontroleerde trial in het Maastricht Universiteit Medisch Centrum in Maastricht, waarin we het preventieve effect van een meerlaagse foam dressing op het sacrum hebben onderzocht. Deze studie werd vroegtijdig beëindigd nadat 244 patiënten met een verhoogd risico (Prepurse score $\geq 21$ of een Braden score $<19$ ) op het ontwikkelen van decubitus waren geïncludeerd. Deze studie is om een aantal reden vroegtijdig beëindigd: Allereerst was er een lage incidentie van sacrale decubitus in deze patiënten groep. Slechts 11 patiënten ontwikkelde decubitus op het sacrum, vier in de dressing groep (3.4\%) en zeven in de controle groep (5.3\%). Slechts vier patiënten ontwikkelde een decubitus categorie 2 of hoger. Daarnaast zagen wij een groot aantal problemen ten gevolge van de dressing. De dressing bleef namelijk niet plakken in 62 van de 117 patiënten in de dressing groep en moest vroegtijdig worden vervangen. Als laatste bleek deze methode niet kosten effectief te zijn. Uit deze studie kunnen we concluderen dat het gebruikt van deze dressing niet effectief was in deze setting. De gebruikte risicoscore lijsten (Prepurse score en Braden score) bleken onvoldoende in staat het risico op decubitus in te schatten. Daarom zou een mogelijk vervolgonderzoek gebruikt moeten maken van een patiënten populatie met een hoger risico op decubitus, alvorens deze dressings aantoonbaar van toegevoegde waarde zouden kunnen zijn.

Hoofdstuk 8 bevat de algemene discussie en aanbevelingen van dit proefschrift. 



\section{Valorisation addendum}





\section{Valorisation addendum}

\section{Social and economic relevance}

Pressure ulcers are a major problem and has a negative influence on health related quality of life. Prevalence rates are up to $9.6 \%$ in acute care settings and $6.1 \%$ in chronic care settings in the Netherlands. In addition, prevalence numbers of pressure ulcers are regarded as an important indicator of quality of care, because most pressure ulcers are considered to be avoidable. Although, not all pressure ulcers are actually avoidable, the occurrence of a pressure ulcer is often blamed on the lack of preventive measures carried out by healthcare workers. ${ }^{1}$ Therefore, prevention of pressure ulcers remain a major burden for hospitals and health care organizations.

The financial impact is high, the United States (US) Agency for Healthcare Research and Quality estimate the total costs in the US between $\$ 9.1$ billion and \$11.6 billion annually, with individual treatment cost ranges between $\$ 20.900$ to $\$ 151.700$ per pressure ulcer. Not only are pressure ulcers associated with an increase in healthcare costs, they also have a negative impact on social and psychological aspects of quality of life, because they are associated with severe pain, and discomfort in patients. Subsequently, they are also associated with longer hospital stay, co-morbidities and mortality. ${ }^{2}$ Research aimed at the prevention of pressure ulcers will further improve quality of life, morbidity and mortality in patients at risk for pressure ulcers.

One of the aims of this thesis was to improve quality of care, quality of life and financial outcomes. Therefore, we carried out a randomized controlled trial in high risk patents in the Maastricht University Medical Centre (MUMC) and investigated the effect of a multi-layered foam dressings in the prevention of sacral pressure ulcers. To address both quality of life and financial outcomes, patient related experiences, adverse device effects and economic evaluations were also reported.

Subsequently, we tried to regain more insight in the development of pressure ulcers and used these insights to test innovative solutions to prevent pressure ulcers by developing a new model and test this in healthy volunteers.

\section{Target population}

The results of this thesis are relevant for other researchers who are focussing on the pathophysiology and biomechanics of pressure ulcers. It offers more information about potential biomarkers such as the cutaneous microbiome in order to predict pressure ulcer risk in patients. The newly developed shear model can be used by other researchers to further investigate the effect of shear force on skin viability in patients. Further, it is also useful for healthcare companies whose profession it is to develop new materials, or optimize dressing design to prevent pressure ulcers in patients.

At last, the result of this thesis could be helpful for care providers who are implementing new pressure ulcer preventive strategies. 


\section{Innovation and future}

This thesis present new and innovative techniques to study the development and prevention of pressure ulcers. First, a new model was designed and validated which enables us to apply shear force on skin in humans and measure its effects by using IL$1 \alpha$, lactate and reactive hyperaemic skin responses. It is the first to describe that the reactive hyperaemic skin response and IL-1 $\alpha$ is increased when shear force is added to pressure.

The prevention of pressure ulcers by focussing on the effect of shear force on skin viability is innovative, because most preventive interventions are focused on pressure redistribution. Developing and testing shear reducing materials is a challenge for healthcare companies, due to the lack of a validated method to apply shear force and the lack of robust measurement techniques. With the help of our newly developed model, we were the first to prove the protective effect of three commercially available against shear forces in vivo. Further, we were able to prove that multilayered dressings performed better than the single-layered foam dressing. The optimal dressing design to prevent pressure ulcers remains unknown. Therefore, future laboratory studies are necessary to determine the ideal properties of preventive dressings. In addition, their clinical relevance and financial efficiency should be tested in randomized controlled trials in several groups of patients with a high incidence of pressure ulcers.

Another innovative aspect was the relation between pressure ulcers and the cutaneous microbiome. This thesis proved that the cutaneous microbiome of the unaffected skin is different compared with those without pressure ulcers. This offers new possibilities in patients at risk for pressure ulcers. First, the cutaneous microbiome could possibly be used as a biomarker to predict pressure ulcer risk in patients. Second, modulating the human microbiome have shown potential in prevention and treatment of several diseases. ${ }^{3,4}$ However, these potential possibilities should be further investigated in future prospective cohort studies before it could be implemented in daily practice. 


\section{References}

1. Black JM, et al. Pressure ulcers: avoidable or unavoidable? Results of the National Pressure Ulcer Advisory Panel Consensus Conference. Ostomy Wound Manage. 2011;57(2):24-37.

2. Bates-Jensen BM. Quality indicators for prevention and management of pressure ulcers in vulnerable elders. Ann Intern Med. 2001;135(8 Pt 2):744-751.

3. Turnbaugh PJ, et al. The human microbiome project. Nature. 2007;449(7164):804-810.

4. Grice EA, Segre JA. The skin microbiome. Nat Rev Microbiol. 2011;9(4):244-253. 

Dankwoord 



\section{Dankwoord}

\section{'Honor est in honorante'- MHD Praetor}

En dan nu het stuk wat eigenlijk als enige echte gelezen wordt. Tevens het stuk waar ik al naar uitkeek voordat ik überhaupt was begonnen met het schrijven van dit proefschrift: Het dankwoord!

Allereerst wil ik alle patiënten en vrijwilligers bedanken die hebben meegewerkt aan het onderzoek. Zonder jullie mee werking aan dit proefschrift had ik helemaal niets op papier kunnen zetten.

Mijn grootste dank gaat uit naar mijn promotoren:

Professor Bouvy, beste Nicole, het zal niet altijd makkelijk voor je zijn geweest om je te verdiepen in een onderwerp waarin je op wetenschappelijk gebied niet veel mee in aanraking bent geweest. Je stond altijd voor meer dan 100 procent achter mijn ideeën en vond het schitterend als ik weer met een wazige schets van mijn model aankwam. Hierdoor heb ik dit kunnen uitwerken en om kunnen zetten in dit proefschrift. Daarnaast lukte het je altijd weer om mij te motiveren en het gevoel te geven dat alles wel goed zou komen. Je was buiten dat altijd geïnteresseerd in hoe het met me ging, waar ik op vakantie was geweest en allerlei andere zaken die niets met dit onderzoek te maken hadden. Bedankt voor alle steun de afgelopen jaren (en nog steeds) en veel succes met het verder begeleiden van nieuwe wetenschappers. Ik zal onze samenwerking in ieder geval erg missen!

Professor Poeze, beste Martijn, je enthousiasme voor wetenschap werkt aanstekelijk. Je scherpe blik en kritische commentaren hebben mijn werk duidelijk verbeterd. De afwisseling met flauwe grappen zeker ook. Ook ben je van onschatbare waarde geweest in het behalen van de Kootstra Talent Fellowship. Bedankt voor je hulp bij het maken van dit boekje.

Ik wil via deze weg een aantal mensen bedanken die erg belangrijk waren in het ontwikkelen van het shear model en mij de eerste stappen binnen het onderzoek en de biomechanica van de huid hebben bijgebracht: Professor Oomens, Professor Bader, Professor Schoonhoven, bedankt voor de ondersteuning, de waardevolle gesprekken en de bijdrage aan diverse artikelen.

Ook wil ik graag Professor Schaper bedanken. Beste Nicolaas, ook al was $u$ initieel niet betrokken bij mijn onderzoek, de deur stond altijd voor mij open om langs te komen. Hieruit volgde interessante discussie over de fysiologie en pathofysiologie van allerlei processen in de huid. Uiteindelijk heeft dit heeft geleid tot een prettige samenwerking en wie weet kunnen wij deze in de toekomst ooit nog verder uitbouwen. 
Daarnaast wil ik John Penders bedanken voor zijn hulp en inzet bij de microbioom studie. Nogmaals gefeliciteerd met het behalen van de Vidi beurs en succes met je verdere wetenschappelijke carrière.

Een zeer belangrijke bijdrage aan dit proefschrift is geleverd door alle co-auteurs: Zita, Sander, Brigitte Essers, Jos Kessels, dr. Piatkowski, professor van der Hulst.

Verder gaat mijn dank uit naar de research nurses die me hebben bijgestaan bij het uitvoeren van het klinisch onderzoek.

Uiteraard kunnen mijn collega's van het chirurgisch lab niet ontbreken in mijn dankwoord.

Mijn kamergenoten: Evelien, Claire, Anne-Claire, Leontine, Annemarie

Wat een kippenhok, een kamer vol met vrouwen, maar ik heb ontzettend veel geleerd van jullie. Ik weet nu bijvoorbeeld wat 'pums' zijn, dat posters van mooie vrouwen of chocolade niet veilig zijn bij jullie, en dat het date leven van een vrijgezelle vrouw helemaal niet zo spannend is. In ieder geval bedankt voor de gezellige tijd, ik heb er van genoten! Trouwens Evelien, als je dit stukje ook wilt kunnen lezen, kun je misschien aan Leontien vragen om dit vertalen naar het Limburgs. Ik heb gehoord dat ze daar ondertussen erg bedreven in is.

Annemarie jou wil ik nog extra bedanken. Als de enige echte 'ELISA queen' heb je mij wegwijs gemaakt in het lab en allerlei vaardigheden bijgebracht. Ook is mijn dank groot voor je hulp bij het analyseren van mijn samples.

Mo, als echte Eindhovenaar en die-hard PSV hooligan hadden we al snel een klik. Regelmatig was ik in je kamer te vinden om te luisteren naar je mooie verhalen, complot theorieën en die goeie ouwe tijd. Dit maakte het werken in het lab altijd weer één groot spektakel. Toendertijd moeizame tijden als PSV fan, maar nu zijn we gelukkig weer aantal kampioenschappen verder en staan we uiteraard weer stijf bovenaan Ge bent unne mooi mens!

Duivenmelker Dennis, Bas, Dirkster, de enige andere mannen van het lab. Tenminste, de enige mannen die altijd in waren voor een leuke grap, voetbalcoach spelen of uitgebreid te lunchen. Jullie onzin heeft me zeker een jaar vertraging opgeleverd, maar de periode op het lab wel erg aangenaam gemaakt.

Verder ook dank voor alle andere collega's van het lab: Professor Olde-Damink, Livia, Audrey, David, Dirk, Frans, Inca, Jungfang, Martijn, Martine, Irene-Fleur, Scarface (Eddy), de Sherriff van Einighausen (Hans), Jacqueline, Frank, Martine, Kim, Victor. Bedankt voor de gezelligheid en met name de vrijdag middag borrels in de Thembi. Ook de wintersport was er één om nooit te vergeten. 
Ook wil ik alle collega's bedanken waarmee ik de afgelopen jaren in de kliniek heb mogen samenwerken. In het bijzonder mijn directe collega's van de chirurgie en orthopedie van het Catharina ziekenhuis in Eindhoven.

Tot slot wil ik nog een aantal mensen bedanken die ook buiten werk om een grote rol hebben gespeeld in de afgelopen periode.

Sander en Michiel, ontzettende paranimfen! Als huisgenoten, teamgenoten, dispuut genoten en kipzonen zijn jullie vrienden die al betrokken waren vanaf het eerste uur. Ik vind het dan ook ontzettend mooi dat jullie tijdens de verdediging achter mij zullen staan.

Dennis en Niels, ouwe troubadours! Het liefste had ik gezien dat jullie ook achter mij op het podium zouden staan. Helaas heb ik dit niet kunnen doen gezien jullie voorkeur voor die voetbalclub uit Amsterdam. Desalniettemin zijn jullie altijd in voor mooie avonturen en andere gekkigheden. Ik stel dan ook voor dat we weer snel op pad gaan naar die mooie bossen van het pittoreske plaatsje 'Grüne'.

De Heeren van MHD Praetor. Allereerst bedankt voor alle mooie momenten in de kroeg en buiten de studie om. Daarnaast stonden jullie altijd voor me klaar als er weer vrijwilligers nodig waren om het model te ontwikkelen en te testen.

In het bijzonder wil ik Sander bedanken, mooi dat je als student mee hebt geholpen en je kwaliteiten heb laten zien in het lab.

Tegen de mannen van Entrepreneur rest mij slechts één woord: olé!

Papa, Mama, Jeroen en Noortje. Niet direct betrokken, maar altijd geïnteresseerd in alles waar ik me mee bezig hield. Vanaf dat ik klein ben hebben jullie mij altijd gestimuleerd om interesse te tonen voor alles wat leeft en beweegt. Bedankt voor de onvoorwaardelijke steun de afgelopen jaren. En ik beloof dat nu het proefschrift klaar is, ik vaker mee zal gaan op weekenden naar Zeeland.

Lieve Meike, al een eeuwigheid bij elkaar, maar elke dag blijft één groot feest. Ook je hulp bij mijn proefschrift onderschat ik zeker niet: opmaak regelen, figuren checken en teksten bekijken. Daarnaast ben je van onschatbare waarde bij de organisatie van mijn promotie dag zelf. Hopelijk wordt de toekomst net zo mooi en blijven we leuke dingen doen. Ik kijk nu al weer uit naar de volgende verre reis die we gaan maken. Ik hou van je! 

Curriculum Vitae 



\section{Curriculum Vitae}

Luuk Albert de Wert werd op 2 november 1986 geboren in Eindhoven. Hier ging hij naar het van Maerlant Lyceum en behaalde hij ook zijn VWO diploma. Aansluitend is hij geneeskunde gaan studeren aan de Universiteit van Maastricht. Tijdens zijn studie heeft hij onder andere stages gelopen in het Rhamatibodi Hospital (Bangkok Thailand) en Kasturba Medical College (Manipal, India). Gedurende zijn wetenschappelijke stage bij de chirurgie in het Maastricht

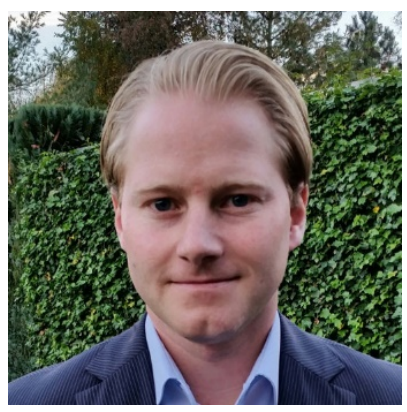
Universitair Medisch Centrum heeft hij een promotietraject opgezet onder begeleiding van prof. dr. N.D. Bouvy en prof. dr. M. Poeze. Na het behalen van zijn artsenbul is hij gaan werken als promovendus op de afdeling NUTRIM school of Nutrition and Translational Research in Metabolism verbonden aan de Universiteit van Maastricht. Enkele van zijn onderzoeken zijn gepresenteerd op internationale congressen in Londen en Madrid. Daarnaast is hij voor zijn onderzoek beloont met de Kootstra Talent Fellowship (MUMC) en zat hij in de organisatie van het Pélerin artsassistenten symposium. Nadien heeft hij klinische ervaring opgedaan als arts-assistent binnen de algemene chirurgie en orthopedie. 
